\title{
The $\eta$-invariant, Maslov index, and spectral flow for Dirac-type operators on manifolds with boundary
}

\author{
Paul Kirk and Matthias Lesch
}

\begin{abstract}
Several proofs have been published of the $\bmod \mathbb{Z}$ gluing formula for the $\eta$-invariant of a Dirac operator. However, so far the integer contribution to the gluing formula for the $\eta$-invariant is left obscure in the literature. In this article we present a gluing formula for the $\eta$-invariant which expresses the integer contribution as a triple index involving the boundary conditions and the Calderón projectors of the two parts of the decomposition. The main ingredients of our presentation are the ScottWojciechowski theorem for the determinant of a Dirac operator on a manifold with boundary and the approach of Brüning-Lesch to the $\bmod \mathbb{Z}$ gluing formula.

Our presentation includes careful constructions of the Maslov index and triple index in a symplectic Hilbert space. As a byproduct we give intuitively appealing proofs of two theorems of Nicolaescu on the spectral flow of Dirac operators.

As an application of our methods, we carry out a detailed analysis of the $\eta$ invariant of the odd signature operator coupled to a flat connection using adiabatic methods. This is used to extend the definition of the Atiyah-Patodi-Singer $\rho$-invariant to manifolds with boundary. We derive a "non-additivity" formula for the AtiyahPatodi-Singer $\rho$-invariant and relate it to Wall's non-additivity formula for the signature of even-dimensional manifolds.
\end{abstract}

\section{Contents}

1. Introduction 2

2. Dirac operators on manifolds with boundary and the self-adjoint Fredholm Grassmannian 4

3. The $\eta$-invariant and spectral flow 11

\begin{tabular}{lll}
\hline 4. & The Scott-Wojciechowski theorem & 13
\end{tabular}

\begin{tabular}{lll}
\hline 5. Splittings of manifolds and the $\eta$-invariant 1 & 17
\end{tabular}

6. Maslov index and winding number 25

7. Splittings of manifolds and the $\eta$-invariant II 35

8. Adiabatic stretching and applications to the Atiyah-Patodi-Singer $\rho$-invariant 39 References

The first named author gratefully acknowledges the support of the National Science Foundation under grant no. DMS-9971020. The second named author was supported by a Heisenberg fellowship of Deutsche Forschungsgemeinschaft and by the National Science Foundation under grant no. DMS0072551 . 


\section{Introduction}

An intriguing feature of certain spectral invariants is that they behave nicely with respect to cutting and pasting. Such a feature has several advantages, in particular with respect to computations. For example, the index of a Dirac operator behaves additively with respect to gluing of manifolds. This is not surprising due to the locality of the index. For higher spectral invariants (e.g. analytic torsion and the $\eta$-invariant) cutting and pasting properties came as a surprise and proofs are nontrivial. The gluing formula for the $\eta$-invariant has a long history (cf. $[7]$ for a historical account). Basically, there are four different types of proof due to Bunke [9], Wojciechowski [33, 34], Müller [25] and Brüning and Lesch [7]. Bunke's argument was simplified and generalized by Dai and Freed [12].

While the articles [33, 34, 25, 12 contain proofs of the gluing formula only in $\mathbb{R} / \mathbb{Z}$, the original formula of Bunke [9] offers a formula for the integer contribution in terms of indices of certain projections. Unfortunately, these projections are not intrinsically defined and therefore Bunke's formula is difficult to work with. In $\|7\|$ it is shown (though not explicitly stated) that the integer contribution can be expressed as the spectral flow of a naturally defined family of self-adjoint operators.

In the current paper we present another formula for the integer contribution in terms of Calderón projectors. This is very satisfactory from a theoretical point of view since all ingredients of the formula are defined intrinsically. Moreover, using adiabatic techniques our formula can be made rather explicit; we carry out a detailed analysis for the odd signature operator.

Given an appropriate orthogonal projection $P$ in the Hilbert space of sections over the boundary, the domain of a Dirac operator $D$ can be restricted to those sections whose restriction to the boundary lie in the kernel of $P$. Denote the resulting operator $D_{P}$. The self-adjoint Fredholm Grassmannian $\operatorname{Gr}(A)$ (see Definition 2.1) consists of those projections $P$ so that $D_{P}$ is a self-adjoint discrete Fredholm operator. It contains a distinguished element, namely the Calderón projector for the Dirac operator $D$. Denote by $\widetilde{\eta}$ the reduced $\eta$-invariant, $\widetilde{\eta}(D)=(\eta(D)+\operatorname{dim} \operatorname{ker} D) / 2$. Our main result is the following. (See Theorem 5.9, Theorem 7.4, and Lemma 5.1.)

Theorem. Let $D$ be a Dirac operator on the closed manifold $M$ and let $N \subset M$ split $M$ into $M^{+}$and $M^{-}$. Assume that $D$ is in product form $D=\gamma\left(\frac{d}{d x}+A\right)$ in a collar of $N$, with $A$ self-adjoint. Let $P \in \operatorname{Gr}(A)$ and let $P_{t}$ be a smooth path in $\operatorname{Gr}(A)$ from $P$ to the Calderón projector $P_{M^{+}}$for $D$ acting on $M^{+}$. Then

$$
\begin{aligned}
\widetilde{\eta}(D, M) & =\widetilde{\eta}\left(D_{P}, M^{+}\right)+\widetilde{\eta}\left(D_{I-P}, M^{-}\right)+\mathrm{SF}\left(D_{P_{t}}, M^{+}\right)_{t \in[0,1]}+\mathrm{SF}\left(D_{I-P_{t}}, M^{-}\right)_{t \in[0,1]} \\
& =\widetilde{\eta}\left(D_{P}, M^{+}\right)+\widetilde{\eta}\left(D_{I-P}, M^{-}\right)-\tau_{\mu}\left(I-P_{M^{-}}, P, P_{M^{+}}\right) .
\end{aligned}
$$

In particular, taking $P=P_{M^{+}}$,

$$
\widetilde{\eta}(D, M)=\widetilde{\eta}\left(D_{P_{M^{+}}}, M^{+}\right)+\widetilde{\eta}\left(D_{I-P_{M^{+}}}, M^{-}\right) .
$$

In these formulas SF denotes the spectral flow, and $\tau_{\mu}$ refers to a Maslov triple index we define for appropriate triples of projections. We also prove a more general formula, Theorem 5.10, which holds for any boundary conditions $(P, Q)$, rather than the special case $(P, I-P)$. 
It is well-known that spectral flow and $\eta$-invariants are intimately related. It is therefore an interesting feature of our approach that it can be used to give new and conceptually simple proofs of Nicolaescu's formulas for the spectral flow of a family of Dirac operators [26]. (See Theorems 7.5 and 7.6.)

For purposes of computation it is usually convenient to use the positive spectral projection of the tangential operator, $P^{+}$, rather than the Calderón projector as boundary conditions. According to our theorem this requires computing $\mathrm{SF}\left(D_{P_{t}}, M^{+}\right)_{t \in[0,1]}+\mathrm{SF}\left(D_{I-P_{t}}, M^{-}\right)_{t \in[0,1]}$ where $P_{t}$ is a path starting at $P^{+}$and ending at the Calderón projector. In favorable circumstances, such a path (actually its reverse) is obtained by stretching the collar neighborhood of the separating hypersurface. More precisely, replacing $M^{+}$by $M^{+} \cup(N \times[-r, 0])$ gives a continuous path (as $\left.r \rightarrow \infty\right)$ of projections starting at the Calderón projector and limiting essentially to $\mathrm{P}^{+}$. This gives a method to obtain computationally useful splitting formulas, and sheds light on the mechanism of adiabatic stretching.

We carry out this analysis in detail in Section 8 for the odd signature operator. Given a flat connection with holonomy $\alpha$ over an odd-dimensional manifold, we take $D$ to be the odd signature operator in the corresponding flat bundle. The adiabatic limit of the Calderón projectors for $D$ as the collar is stretched is identified in Theorem 8.5 . We use this identification along with the topological invariance of the kernel of $D$ to establish the formula (cf. (8.32)):

$$
\eta(D, M)=\eta\left(D_{P^{+}\left(V_{+, \alpha}\right)}, M^{+}\right)+\eta\left(D_{P^{-}\left(V_{-, \alpha}\right)}, M^{-}\right)+m\left(V_{+, \alpha}, V_{-, \alpha}, \alpha, g\right) .
$$

In this expression $V_{ \pm, \alpha}=\operatorname{im} H^{*}\left(M^{ \pm} ; \mathbb{C}_{\alpha}^{n}\right) \rightarrow H^{*}\left(N ; \mathbb{C}_{\alpha}^{n}\right)$, and $m\left(V_{+, \alpha}, V_{-, \alpha}, \alpha, g\right)$ is a real-valued symplectic invariant which depends only on the subspaces $V_{ \pm, \alpha} \subset$ $H^{*}\left(N ; \mathbb{C}_{\alpha}^{n}\right)$ and a choice of Riemannian metric on the separating hypersurface $N$. The projections $P^{ \pm}\left(V_{ \pm, \alpha}\right)$ are the sum of the positive/negative spectral projections of the tangential operator and the finite-dimensional projection to $V_{ \pm, \alpha}$. In particular if $H^{*}\left(N ; \mathbb{C}_{\alpha}^{n}\right)=0$ the formula simplifies to

$$
\widetilde{\eta}(D, M)=\widetilde{\eta}\left(D_{P^{+}}, M^{+}\right)+\widetilde{\eta}\left(D_{P^{-}}, M^{-}\right) .
$$

These formulae motivate a definition for the $\rho$-invariant of a manifold with boundary, $\rho(X, \alpha, g)$ (Definition 8.17), which is shown to depend only on the smooth structure of $X$, the conjugacy class of the representation $\alpha$, and the choice of Riemannian metric $g$ on $\partial X$. We then prove the following theorem, and discuss its relation to Wall's non-additivity theorem [32] for the signature of even-dimensional manifolds.

TheOREM 8.18. Suppose the closed, odd-dimensional manifold $M$ contains a hypersurface $N$ separating $M$ into $M^{+}$and $M^{-}$. Fix a Riemannian metric $g$ on $N$. Suppose that $\alpha: \pi_{1}(M) \rightarrow U(n)$ is a representation, and let $\tau: \pi_{1}(M) \rightarrow U(n)$ denote the trivial representation. Then

$$
\rho(M, \alpha)=\rho\left(M^{+}, \alpha, g\right)+\rho\left(M^{-}, \alpha, g\right)+m\left(V_{+, \alpha}, V_{-, \alpha}, \alpha, g\right)-m\left(V_{+, \tau}, V_{-, \tau}, \alpha, g\right) .
$$

The paper is organized as follows:

In Section 2 we review the basic facts about Dirac operators on manifolds with boundary and the Grassmannian of their boundary value problems. 
In Section 3 we introduce the $\eta$-invariant and review its basic features. Using the Scott-Wojciechowski Theorem [30] we prove in Section 4 a formula describing the dependence on the choice of boundary condition of the $\eta$-invariant of a Dirac operator on a manifold with boundary (Theorem 4.4).

Section 5 deals with splittings of manifolds. We prove a result on the behavior of the spectral flow under splittings (Corollary 5.6) and the gluing formula for the $\eta$-invariant (Theorem 5.10).

Section 6 contains careful constructions of various forms of the Maslov index for families of self-adjoint projections in a Hermitian symplectic Hilbert space. Conventions must be set to deal with degenerate situations when defining symplectic invariants, and we carefully construct the various invariants consistently and in such a way that they match our choice of convention for the spectral flow.

A byproduct of our considerations are new proofs of (generalizations of) two theorems by Nicolaescu [26] identifying the spectral flow of a family of Dirac operators with a Maslov index involving the Calderón projectors and boundary conditions. These results (Theorem 7.5 for manifolds with boundary and Theorem 7.6 for split manifolds), together with an improvement (Theorem 7.7) of our gluing formula for the $\eta$-invariant which allows more general boundary conditions, are presented in Section 7 .

Finally, in Section 8 we apply our splitting results for the $\eta$-invariant to the special case of the odd signature operator coupled to a flat connection. By making use of the method of adiabatic stretching of the collar of a separating hypersurface and the fact that the dimension of the kernels of these operators are topological, i.e. independent of the Riemannian metric, we obtain a splitting formula for the Atiyah-Patodi-Singer $\rho_{\alpha}$ invariant. The main tool introduced in this section is Theorem 8.5, which gives a precise identification of the adiabatic limit of the Calderón projectors in this setting. We end the paper with an examination of the role adiabatic stretching plays in addition formulas for the $\eta$-invariants of general Dirac operators.

\section{Dirac operators on manifolds with boundary and the self-adjoint Fredholm Grassmannian}

We begin by describing the set-up of Dirac operators on a manifold with boundary.

Let $X$ denote a compact smooth Riemannian manifold with boundary $\partial X$. We fix an identification of a neighborhood of $\partial X$ in $X$ with $\partial X \times[0, \varepsilon)$. Let $E \rightarrow X$ be a complex Hermitian vector bundle and suppose that $D: C^{\infty}(E) \rightarrow C^{\infty}(E)$ is a symmetric Dirac operator, i.e. a symmetric first-order operator whose square is a generalized Laplacian (the square of the leading symbol of $D$ is scalar and given by the metric tensor). The symmetry is measured with respect to the $L^{2}$ inner product; thus we assume that if $\phi_{1}, \phi_{2} \in C^{\infty}(E)$ are supported in the interior of $X$ then

$$
\int_{X}\left\langle D \phi_{1}, \phi_{2}\right\rangle_{E_{x}} d x=\int_{X}\left\langle\phi_{1}, D \phi_{2}\right\rangle_{E_{x}} d x
$$

A Dirac operator satisfies the unique continuation property [5].

In this paper we will deal only with the product case, i.e. we assume that the restriction of $D$ to the collar takes the form $D=\gamma\left(\frac{d}{d x}+A\right)$, where $\gamma: E_{\mid \partial X} \rightarrow E_{\mid \partial X}$ 
is a bundle endomorphism and $A: C^{\infty}\left(E_{\mid \partial X}\right) \rightarrow C^{\infty}\left(E_{\mid \partial X}\right)$ is a first-order self-adjoint elliptic differential operator on the closed manifold $\partial X$ (called the tangential operator) satisfying

$$
\gamma^{2}=-I, \quad \gamma^{*}=-\gamma, \quad \text { and } \quad \gamma A=-A \gamma .
$$

Note that $A$ is assumed to be independent of $x$ for $x \in[0, \varepsilon)$.

The operator $D: C^{\infty}(E) \rightarrow C^{\infty}(E)$ can be extended to an unbounded self-adjoint operator on $L^{2}(E)$ by imposing appropriate boundary conditions. Since $D$ is a first order operator, it can be extended to a bounded operator $H_{1}(E) \rightarrow L^{2}(E)$, where $H_{s}(E)$ denotes the Sobolev space of sections of $E$ with $s$ derivatives in $L^{2}$. Given an orthogonal projection $P: L^{2}\left(E_{\mid \partial X}\right) \rightarrow L^{2}\left(E_{\mid \partial X}\right)$ define $D_{P}$ to be $D$ acting on the domain

$$
\mathscr{D}\left(D_{P}\right):=\left\{\phi \in L^{2}(E) \mid \phi \in H_{1}(E) \text { and } P\left(\phi_{\mid \partial X}\right)=0\right\} \subset L^{2}(E) .
$$

We will consider the operators $D_{P}$ for a certain class of projections $P$ which we now introduce. Let

$$
P_{>0}: L^{2}\left(E_{\mid \partial X}\right) \longrightarrow L^{2}\left(E_{\mid \partial X}\right)
$$

denote the positive spectral projection for the self-adjoint tangential operator $A$ : $C^{\infty}\left(E_{\mid \partial X}\right) \rightarrow C^{\infty}\left(E_{\mid \partial X}\right)$; thus if $\left\{\psi_{\lambda}\right\}$ is a basis of $L^{2}\left(E_{\mid \partial X}\right)$ with $A \psi_{\lambda}=\lambda \psi_{\lambda}$, then $P_{>0}\left(\sum a_{\lambda} \psi_{\lambda}\right)=\sum_{\lambda>0} a_{\lambda} \psi_{\lambda}$.

Definition 2.1. Define the self-adjoint Fredholm Grassmannian $\operatorname{Gr}(A)$ to be the set of maps $P: L^{2}\left(E_{\mid \partial X}\right) \rightarrow L^{2}\left(E_{\mid \partial X}\right)$ so that

(1) $P$ is pseudo-differential of order 0 ,

(2) $P=P^{*}, P^{2}=P$, i.e. $P$ is an orthogonal projection,

(3) $\gamma P \gamma^{*}=I-P$,

(4) $\left(P_{>0}, P\right)$ form a Fredholm pair, that is,

$$
P_{>0 \mid \operatorname{im} P}: \operatorname{im} P \rightarrow \operatorname{im} P_{>0}
$$

is Fredholm.

The Grassmannian $\operatorname{Gr}(A)$ is topologized using the norm topology on bounded operators.

REMARK 2.2.

1. We note that a $P \in \operatorname{Gr}(A)$ also acts as a (non-orthogonal) projection in the Sobolev space $H_{s}(E)$ for all $s \in \mathbb{R}$. This follows from (I).

2. We obtain the same Grassmannian if we replace $P_{>0}$ in (41) by any pseudodifferential orthogonal projection $Q$ such that $P_{>0}-Q$ is smoothing. This follows immediately from the following general fact:

Let $P, Q, R$ be orthogonal projections in the Hilbert space $H$ such that $Q-R$ is compact. Then $(P, Q)$ is a Fredholm pair if and only if $(P, R)$ is a Fredholm pair. 
This fact can be seen as follows: by [3, Prop. 3.1] $(P, Q)$ is Fredholm if and only if $\pm 1 \notin \operatorname{spec}_{\mathrm{ess}}(P-Q)$. Since $Q-R$ is compact this is equivalent to $\pm 1 \notin \operatorname{spec}_{\mathrm{ess}}(P-R)$. Applying again [3, Prop. 3.1] the latter is the case if and only if $(P, R)$ is Fredholm.

If $P \in \operatorname{Gr}(A)$, then $D_{P}$ is self-adjoint, Fredholm, and has compact resolvent; in particular its spectrum is discrete and each eigenvalue has finite multiplicity. These facts follow since $(D, P)$ is a well-posed boundary value problem in the sense of $\mathrm{R}$. T. Seeley [31]. A general reference for boundary value problems for Dirac type operators is the monograph [5]. A different approach is presented in [8, 6].

It will be necessary to consider a more restricted class of projections, those that differ from $P_{>0}$ by a smoothing operator. Define $\operatorname{Gr}_{\infty}(A) \subset \operatorname{Gr}(A)$ by

$$
\operatorname{Gr}_{\infty}(A)=\left\{P \in \operatorname{Gr}(A) \mid P-P_{>0} \text { is a smoothing operator }\right\} .
$$

Again, in (2.2) we can replace $P_{>0}$ by any pseudo-differential orthogonal projection $Q$ such that $P_{>0}-Q$ is smoothing.

The projection $P_{>0}$ does not lie in $\operatorname{Gr}(A)$ unless ker $A=0$, since the third condition does not hold for $P=P_{>0}$ if $\operatorname{ker} A \neq 0$. It is convenient to specify a finite rank perturbation of $P_{>0}$ which does lie in $\operatorname{Gr}(A)$.

Notice that $\gamma$ leaves ker $A$ invariant. It is well-known that since $(\partial X, A)$ "bounds" $(X, D)$, the $i$ and $-i$ eigenspaces of $\gamma$ acting on $\operatorname{ker} A$ have the same dimension [27, Chap. XVII]. This implies that there are subspaces $L \subset \operatorname{ker} A$ satisfying $\gamma(L)=$ $L^{\perp} \cap$ ker $A$ (such subspaces are called Lagrangian subspaces; see Definition 2.8 below). Given a Lagrangian subspace $L \subset \operatorname{ker} A$ define

$$
P^{+}(L)=\operatorname{proj}_{L}+P_{>0} .
$$

Then $P^{+}(L)$ differs from $P_{>0}$ by the projection onto $L$, a subspace of ker $A$, which consists only of smooth functions. Since $P_{>0}$ is a 0th order pseudo-differential projection, so is $P^{+}(L)$. It is straightforward to check that $P^{+}(L) \in \operatorname{Gr}_{\infty}(A)$.

We call $P^{+}(L)$ the Atiyah-Patodi-Singer projection corresponding to the Lagrangian subspace $L$. Notice that $P^{+}(L)$ depends only on the tangential operator $A$ and the choice of $L$; in particular it is unchanged if $D$ is altered in the interior of $X$.

There is a canonical projection in $\operatorname{Gr}(A)$ determined by the operator $D$ which will play a special role in what follows, namely the Calderón projector $P_{X}$. It is defined as the orthogonal projection onto the Cauchy data space

$$
L_{X}:=r\left(\operatorname{ker} D: H_{1 / 2}(E) \longrightarrow H_{-1 / 2}(E)\right) \subset L^{2}\left(E_{\mid \partial X}\right) .
$$

Here $r$ denotes the restriction to the boundary. The trace operator $r$ is a priori only defined on $H_{s}(E)$ for $s>1 / 2$ but one can show that $r$ defines a bounded map from the $H_{1 / 2}$-kernel of $D$ into $L^{2}\left(E_{\mid \partial X}\right)$ (see [5] for a proof).

The Calderón projector $P_{X}=\operatorname{proj}_{L_{X}}$ lies in $\operatorname{Gr}_{\infty}(A)$ [29, Prop. 2.2], [17, Prop. 4.1]. The unique continuation property for $D$ implies that

$$
r:\left(\operatorname{ker} D: H_{1 / 2}(E) \longrightarrow H_{-1 / 2}(E)\right) \longrightarrow L^{2}\left(E_{\mid \partial X}\right)
$$


is injective, so that to any vector $x$ in the image of $P_{X}$ we can assign a unique solution to $D \phi=0$ on $X$ with $\phi \in H_{1 / 2}$ and $r(\phi)=x$. This makes it possible to identify the kernel of $D$ with boundary condition given by a projection $P$ and the intersection of the Cauchy data space with the kernel of $P$, as in the following lemma.

Lemma 2.3. Let $P \in \operatorname{Gr}(A)$. Then

$$
\operatorname{ker} P_{\mid \operatorname{im} P_{X}}=\operatorname{im} P_{X} \cap \operatorname{ker} P=\gamma\left(\operatorname{ker} P_{X}\right) \cap \operatorname{ker} P
$$

and this space is isomorphic to the kernel of $D_{P}$. Thus $D_{P}$ is invertible if and only if $\operatorname{im} P_{X} \cap \operatorname{ker} P=0$. In particular $D_{P_{X}}$ is invertible.

Proof. If $\phi \in \operatorname{ker} D$, then by definition the restriction of $\phi$ to the boundary of $X$ lies in the image of the Calderón projector $P_{X}$. In particular, if $\phi \in \operatorname{ker} D_{P}$, then the restriction of $\phi$ to the boundary lies in the intersection of ker $P$ and the image of $P_{X}$. The unique continuation property for $D$ implies that this intersection is exactly the kernel of $D_{P}$, i.e. the kernel of $D_{P}$ is isomorphic to ker $P_{\mid \operatorname{im} P_{X}}$.

As a discrete self-adjoint operator, $D_{P}$ is invertible if and only if ker $D_{P}=\{0\}$. Moreover, $P_{X}$ is a self-adjoint projection satisfying the equation $\gamma P_{X} \gamma=-\left(I-P_{X}\right)$. Thus im $P_{X}=\operatorname{ker}\left(I-P_{X}\right)=\gamma\left(\operatorname{ker} P_{X}\right)$.

In a rough sense the Atiyah-Patodi-Singer projection $P^{+}(L)$ and the Calderón projector $P_{X}$ are opposites: $P^{+}(L)$ is determined entirely by the boundary data, i.e. the tangential operator $A$ acting on $\partial X$ (and the choice of $L$ ), whereas $P_{X}$ depends on all of $X$ and $D$.

For future reference we note the following special case of a result due to K. Wojciechowski.

Proposition 2.4. The Grassmannians $\operatorname{Gr}(A), \mathrm{Gr}_{\infty}(A)$ are path connected. For a fixed $P \in \operatorname{Gr}_{\infty}(A)$ (resp. $\operatorname{Gr}(A)$ ) the space $\left\{Q \in \operatorname{Gr}_{\infty}(A) \mid \operatorname{ker} Q \cap \operatorname{im} P=0\right\}$ (resp. $\{Q \in \operatorname{Gr}(A) \mid \operatorname{ker} Q \cap \operatorname{im} P=0\})$ is path connected.

Remark 2.5. This result could also be proved using Proposition 6.5 below (resp. its analog for pseudo-differential Grassmannians) and properties of the unitary group.

Proof. The first statement is a special case of [15, Appendix B], where the homotopy groups of $\operatorname{Gr}_{\infty}(A)$ and $\operatorname{Gr}(A)$ are computed. The path connectedness of $\left\{Q \in \operatorname{Gr}_{\infty}(A) \mid \operatorname{ker} Q \cap \operatorname{im} P=0\right\}$ was proved in Proposition 5.1 of [30]. The path connectedness of $\{Q \in \operatorname{Gr}(A) \mid \operatorname{ker} Q \cap \operatorname{im} P=0\}$ can be proved along the same lines: if $\operatorname{ker} Q \cap \operatorname{im} P=0$ then $\|Q-P\|<1$ and hence $Q_{s}:=Z_{s} P Z_{s}^{-1}, 0 \leq s \leq 1$, where $Z_{s}:=I+s(Q-P)(2 P-I)$, is a path in $\operatorname{Gr}(A)$ connecting $P$ and $Q$ (cf. e.g. [6], Sec. $3])$.

Notice that $\operatorname{Gr}(A)$ and $\operatorname{Gr}_{\infty}(A)$ can also be defined by replacing $P_{>0}$ by $P^{+}(L)$ or $P_{X}$ in the fourth condition defining $\operatorname{Gr}(A)$, and in (2.2). 
We next discuss two alternative perspectives on the Grassmannian $\operatorname{Gr}(A)$, identifying this space with the space of certain unitary operators on a Hilbert space, and also with certain Lagrangian subspaces of a symplectic Hilbert space.

The bundle endomorphism $\gamma: E_{\mid \partial X} \rightarrow E_{\mid \partial X}$ induces a decomposition of $E_{\mid \partial X}=$ $E_{i} \oplus E_{-i}$ into the $\pm i$ eigenbundles and consequently we get a decomposition of $L^{2}\left(E_{\mid \partial X}\right)$ into the $\pm i$ eigenspaces,

$$
L^{2}\left(E_{\mid \partial X}\right)=L^{2}\left(E_{i}\right) \oplus L^{2}\left(E_{-i}\right)=: \mathscr{E}_{i} \oplus \mathscr{E}_{-i}
$$

Given $P \in \operatorname{Gr}(A)$, write

$$
P=\frac{1}{2}\left(\begin{array}{ll}
A & B \\
C & D
\end{array}\right)
$$

with respect to the decomposition (2.5). Then $P=P^{*}$ implies $C=B^{*}$. The conditions $\gamma P \gamma^{*}=I-P$ and $\gamma^{*}=-\gamma$ imply that $A=D=I$, and the condition $P^{2}=P$ implies that $B B^{*}=I=B^{*} B$. This proves the first part of the following lemma.

Lemma 2.6. If $P \in \operatorname{Gr}(A)$, then with respect to the decomposition (2.5), $P$ can be written in the form

$$
P=\frac{1}{2}\left(\begin{array}{cc}
I & T^{*} \\
T & I
\end{array}\right)
$$

where $T$ is a 0th order pseudo-differential isometry from $\mathscr{E}_{i}$ onto $\mathscr{E}_{-i}$. Conversely, given such an isometry $T$, then

$$
\frac{1}{2}\left(\begin{array}{cc}
I & T^{*} \\
T & I
\end{array}\right)
$$

is a pseudo-differential projection satisfying (1), (2), (3) of Definition 2.1.

Given

$$
P=\frac{1}{2}\left(\begin{array}{cc}
I & T^{*} \\
T & I
\end{array}\right), Q=\frac{1}{2}\left(\begin{array}{cc}
I & S^{*} \\
S & I
\end{array}\right),
$$

satisfying (11), (2), (3) of Definition 2.1, then:

(1) $(P, Q)$ form a Fredholm pair if and only if $-1 \notin \mathrm{spec}_{\mathrm{ess}} T^{*} S$,

(2) $(P, Q)$ is invertible if and only if $-1 \notin \operatorname{spec} T^{*} S$,

(3) $\operatorname{ker} P \cap \operatorname{im} Q$ is canonically isomorphic to $\operatorname{ker}\left(I+T^{*} S\right)$,

(4) $P-Q$ is smoothing if and only if $T^{*} S-I$ is smoothing.

In particular, if $Q=P^{+}(L)$ for some Lagrangian $L \subset \operatorname{ker} A$, then $P \in \operatorname{Gr}(A)$ if and only if $-1 \notin \operatorname{spec}_{\mathrm{ess}} T^{*} S$.

Proof. The first part was proved above. Since $S^{*} S=I=S S^{*}$, any element in $L^{2}\left(E_{\mid \partial X}\right)=\mathscr{E}_{i} \oplus \mathscr{E}_{-i}$ can be written in the form $\left(\begin{array}{c}x \\ S y\end{array}\right)$ for $x, y \in \mathscr{E}_{i}$. Since

$$
Q\left(\begin{array}{c}
x \\
S y
\end{array}\right)=\frac{1}{2}\left(\begin{array}{c}
x+y \\
S(x+y)
\end{array}\right)
$$


it follows that $\operatorname{im} Q=\left\{\left(\begin{array}{c}x \\ S x\end{array}\right) \mid x \in \mathscr{E}_{i}\right\}$. Thus the restriction of $P$ to the image of $Q$ is

$$
P\left(\begin{array}{c}
x \\
S x
\end{array}\right)=\frac{1}{2}\left(\begin{array}{c}
\left(I+T^{*} S\right) x \\
T\left(I+T^{*} S\right) x
\end{array}\right)
$$

It follows that $(P, Q)$ is Fredholm (i.e. $P$ restricts to a Fredholm operator on the image of $Q$ ) if and only if $I+T^{*} S$ is Fredholm, which occurs precisely when -1 is not in the essential spectrum of $T^{*} S$. Similarly $(P, Q)$ is invertible (i.e. the restriction of $P$ to the image of $Q$ defines an isomorphism onto the image of $P$ ) if and only if -1 is not in the spectrum of $T^{*} S$. The same argument also shows (3).

Finally, since

$$
P-Q=\left(\begin{array}{cc}
0 & T^{*}-S^{*} \\
T-S & 0
\end{array}\right)
$$

$P-Q$ is smoothing if and only if $T-S$ is smoothing. Here we use that the projections $\frac{1}{2 i}(i \pm \gamma)$ onto $\mathscr{E}_{ \pm i}$ are differential operators of order 0 . Since $T, S$ are pseudo-differential and unitary the operator $T-S$ is smoothing if and only if $T^{*} S-I$ is smoothing.

Let $\mathscr{U}\left(\mathscr{E}_{i}, \mathscr{E}_{-i}\right)$ denote the set of unitary isomorphisms from $\mathscr{E}_{i}$ to $\mathscr{E}_{-i}$. Then $P \mapsto T$ defines a map

$$
\Phi: \operatorname{Gr}(A) \longrightarrow \mathscr{U}\left(\mathscr{E}_{i}, \mathscr{E}_{-i}\right)
$$

i.e.,

$$
P=\frac{1}{2}\left(\begin{array}{cc}
I & \Phi(P)^{*} \\
\Phi(P) & I
\end{array}\right) .
$$

More abstractly, consider the group $\mathscr{U}$ of unitary pseudo-differential isomorphisms $\mathscr{E}_{i} \rightarrow \mathscr{E}_{i}$. Let

$$
\mathscr{U}_{\text {Fred }}=\left\{U \in \mathscr{U} \mid-1 \notin \operatorname{spec}_{\text {ess }} U\right\},
$$

and

$$
\mathscr{U}_{\infty}=\left\{U \in \mathscr{U}_{\text {Fred }} \mid U-I \text { is a smoothing operator }\right\} .
$$

Then given any $P \in \operatorname{Gr}_{\infty}(A)$, the map

$$
U \mapsto \frac{1}{2}\left(\begin{array}{cc}
I & (\Phi(P) U)^{*} \\
\Phi(P) U & I
\end{array}\right)
$$

defines homeomorphisms

$$
\mathscr{U}_{\text {Fred }} \longrightarrow \operatorname{Gr}(A)
$$

and

$$
\mathscr{U}_{\infty} \longrightarrow \operatorname{Gr}_{\infty}(A)
$$

Another useful description of $\operatorname{Gr}(A)$ and $\operatorname{Gr}_{\infty}(A)$ is in terms of Lagrangian subspaces. 
Lemma 2.7. Let $(H,\langle\rangle$,$) be a separable complex Hilbert space and \gamma: H \rightarrow H$ an isomorphism satisfying $\gamma^{2}=-I, \gamma^{*}=-\gamma$. Then there exists a subspace $L \subset H$ such that $\gamma(L)=L^{\perp}$ if and only if $\operatorname{dim} \operatorname{ker}(\gamma-i)=\operatorname{dim} \operatorname{ker}(\gamma+i)$.

Proof. Suppose $L \subset H$ is a subspace with $\gamma(L)=L^{\perp}$. Then it is easy to check that the orthogonal projections $\pi_{ \pm}: L \rightarrow \operatorname{ker}(\gamma \pm i)$ are isomorphisms and hence $\operatorname{dim} \operatorname{ker}(\gamma+i)=\operatorname{dim} \operatorname{ker}(\gamma-i)$.

Conversely, if $\operatorname{dim} \operatorname{ker}(\gamma+i)=\operatorname{dim} \operatorname{ker}(\gamma-i)$ then let $T: \operatorname{ker}(\gamma-i) \rightarrow \operatorname{ker}(\gamma+i)$ be a unitary isomorphism. Then $L=\left\{\left(\begin{array}{c}x \\ T x\end{array}\right) \mid x \in \operatorname{ker}(\gamma-i)\right\}$ is a subspace satisfying $\gamma(L)=L^{\perp}$.

Definition 2.8. A Hermitian symplectic Hilbert space is a separable complex Hilbert space together with an isomorphism $\gamma: H \rightarrow H$ satisfying $\gamma^{2}=-I, \gamma^{*}=-\gamma$ and such that the $i$ and $-i$ eigenspaces of $\gamma$ have the same dimension (i.e. if $H$ is infinite-dimensional we require that both eigenspaces are infinite-dimensional). The symplectic form is the skew-Hermitian form

$$
\omega(x, y):=\langle x, \gamma y\rangle .
$$

A Lagrangian subspace $L \subset H$ of a Hermitian symplectic Hilbert space is a subspace so that $\gamma(L)=L^{\perp}$. A Lagrangian subspace is automatically closed.

The space $L^{2}\left(E_{\mid \partial X}\right)$ together with the map $\gamma$ is a Hermitian symplectic Hilbert space. The space ker $A$ is a finite-dimensional Hermitian symplectic Hilbert space since $(\partial X, A)$ bounds $(X, D)$.

Given $P \in \operatorname{Gr}(A)$, the kernel of $P$ is a Lagrangian subspace, since ker $P$ is orthogonal to $\gamma($ ker $P)$. Notice that the kernel of $P$ can be expressed as the graph of $-\Phi(P)$,

$$
\operatorname{ker} P=\left\{\left(\begin{array}{c}
x \\
-\Phi(P) x
\end{array}\right) \mid x \in \mathscr{E}_{i}\right\} \subset L^{2}\left(E_{\mid \partial X}\right) .
$$

This gives a third characterization of $\operatorname{Gr}(A)$ as follows. We define $\mathscr{L}$ to be the set of Lagrangian subspaces of $L^{2}\left(E_{\mid \partial X}\right)$ whose associated projections are pseudo-differential of order 0 . The Cauchy data space, $L_{X}$, (the image of the Calderón projector) is a Lagrangian subspace of $L^{2}\left(E_{\mid \partial X}\right)$.

Define

$$
\mathscr{L}_{\text {Fred }}=\left\{L \in \mathscr{L} \mid\left(L, \gamma\left(L_{X}\right)\right) \text { is a Fredholm pair of subspaces }\right\},
$$

and

$$
\mathscr{L}_{\infty}=\left\{L \in \mathscr{L}_{\text {Fred }} \mid \operatorname{proj}_{L}-\operatorname{proj}_{L_{X}} \text { is a smoothing operator }\right\} .
$$

Then we have homeomorphisms

$$
\mathscr{L}_{\text {Fred }} \longrightarrow \operatorname{Gr}(A)
$$

and

$$
\mathscr{L}_{\infty} \longrightarrow \operatorname{Gr}_{\infty}(A)
$$


$\eta$-INVARIANT, MASLOV INDEX, AND SPECTRAL FLOW

The identifications of $\mathscr{L}, \operatorname{Gr}(A)$, and $\mathscr{U}$ are determined by the conditions that

$$
L \in \mathscr{L}_{\text {Fred }}, P \in \operatorname{Gr}(A) \text {, and } T \in \mathscr{U}_{\text {Fred }}\left(\mathscr{E}_{i}, \mathscr{E}_{-i}\right)
$$

correspond if

$$
L=\operatorname{im} P=\text { graph of } T \text { and } T=\Phi(P) .
$$

\section{The $\eta$-invariant and spectral flow}

It was mentioned in the last section that $D_{P}$ is the self-adjoint realization of a wellposed boundary value problem and hence it is a discrete operator in the Hilbert space $L^{2}(E)$. For the discussion of $\zeta$ - and $\eta$-functions we need the more refined analysis of the heat trace of $D_{P}$. The $\zeta$ - and $\eta$-functions of $D_{P}$ are defined, for $\operatorname{Re}(s)>>0$, by

$$
\begin{aligned}
\eta\left(D_{P} ; s\right) & :=\operatorname{tr}\left(D_{P}\left|D_{P}\right|^{-s-1}\right)=\sum_{\lambda \in \operatorname{spec} D_{P} \backslash\{0\}} \operatorname{sign}(\lambda)|\lambda|^{-s}, \\
\zeta\left(D_{P} ; s\right) & :=\operatorname{tr}\left(D_{P}^{-s}\right)=\sum_{\lambda \in \operatorname{spec} D_{P} \backslash\{0\}} \lambda^{-s} \\
& =\frac{1}{2}\left(\zeta\left(D_{P}^{2} ; s / 2\right)+\eta\left(D_{P} ; s\right)\right)+e^{-i \pi s} \frac{1}{2}\left(\zeta\left(D_{P}^{2} ; s / 2\right)-\eta\left(D_{P} ; s\right)\right) .
\end{aligned}
$$

Theorem 3.1. For $P \in \operatorname{Gr}(A)$ the functions $\zeta\left(D_{P} ; s\right), \eta\left(D_{P} ; s\right)$ extend meromorphically to the whole complex plane with poles of order at most 2. If $P \in \operatorname{Gr}_{\infty}(A)$ then $\eta\left(D_{P} ; s\right)$ and $\zeta\left(D_{P} ; s\right)$ are regular at $s=0$. Moreover $\zeta\left(D_{P} ; 0\right)$ is independent of $P \in \operatorname{Gr}_{\infty}(A)$.

That the $\zeta$ - and $\eta$-functions extend meromorphically has been proved in increasing generality in [15], [18], [19], [7], [35], and [17]. The definitive treatment of all wellposed boundary value problems is given in [17]. The proof of the statement about regularity at $s=0$ can be found in [35. The methods of [17 show that the assumption $P \in \mathrm{Gr}_{\infty}(A)$ can be somewhat relaxed [16]. Finally, that $\zeta\left(D_{P} ; 0\right)$ is independent of $P \in \mathrm{Gr}_{\infty}(A)$ is [35, Prop. 0.5].

Definition 3.2. The $\eta$-invariant of $D_{P}, \eta\left(D_{P}\right)$, is defined to be the constant term in the Laurent expansion of $\eta\left(D_{P} ; s\right)$ at $s=0$, i.e.

$$
\eta\left(D_{P} ; s\right)=a s^{-2}+b s^{-1}+\eta\left(D_{P}\right)+O(s) .
$$

We also give a symbol to a convenient normalization of the $\eta$-invariant.

Definition 3.3. The reduced $\eta$-invariant is defined to be

$$
\widetilde{\eta}\left(D_{P}\right)=\left(\eta\left(D_{P}\right)+\operatorname{dim} \operatorname{ker} D_{P}\right) / 2 \text {. }
$$

We continue with a discussion of the spectral flow and its relation to the $\eta$-invariant. Suppose one is given a smooth path of Dirac type operators $D_{t}: C^{\infty}(E) \rightarrow C^{\infty}(E), t \in$ $[0,1]$, over $X$ so that $D_{t}=\gamma\left(\frac{d}{d x}+A_{t}\right)$ on the collar. Choose a smooth path of projections $P_{t}$ so that $P_{t} \in \operatorname{Gr}\left(A_{t}\right)$ for $t \in[0,1]$. Then the family $D_{P}(t):=\left(D_{t}\right)_{P_{t}}$ is in particular a graph continuous family of self-adjoint discrete operators. As a consequence, the eigenvalues of $D_{P_{t}}$ vary continuously (as a general reference see [21]). The spectral 
flow of the family $D_{P}(t)$, which we denote by $\mathrm{SF}\left(D_{P}(t)\right)_{t \in[0,1]}$ or just $\mathrm{SF}\left(D_{P}(t)\right)$, is the integer defined (roughly) to be the difference in the number of eigenvalues that start negative and end non-negative and the number of eigenvalues that start non-negative and end negative (see [5, 11] for a precise definition). Notice that we have chosen a particular convention for dealing with zero eigenvalues. This convention is often called the $(-\varepsilon,-\varepsilon)$ spectral flow in the literature, since it corresponds to intersecting the graph of the spectrum as a function of $t$ with the line from $(0,-\varepsilon)$ to $(1,-\varepsilon)$.

The 1-parameter family of $\eta$-invariants $\eta\left(D_{P}(t)\right) \in \mathbb{R}$ will in general not vary smoothly with respect to $t \in[0,1]$. However, it follows from the work of G. Grubb [17 that the reduction modulo integers of the reduced $\eta$-invariant $\widetilde{\eta}\left(D_{P}(t)\right)$ varies smoothly with $t$. In particular, the real valued function

$$
u \mapsto \int_{0}^{u} \frac{d}{d t}\left(\eta\left(D_{P}(t)\right)\right) d t
$$

is smooth.

In general, given a smooth function $f:[0,1] \rightarrow \mathbb{R} / \mathbb{Z}=S^{1}$, the expression $u \mapsto$ $c+\int_{0}^{u} \frac{d f}{d t} d t$ is just an explicit formula for the unique smooth lift of $f$ to the universal cover $\mathbb{R}$ of $S^{1}$ starting at $c \in \mathbb{R}$. Thus if $f$ and $g$ are (possibly discontinuous) functions from $[0,1]$ to $\mathbb{R}$ so that the reductions of $f$ and $g$ modulo $\mathbb{Z}$ are smooth and agree, then the smooth real-valued functions $u \mapsto \int_{0}^{u} \frac{d f}{d t} d t$ and $u \mapsto \int_{0}^{u} \frac{d g}{d t} d t$ coincide.

LEMma 3.4. Suppose that $D_{t}, t \in[0,1]$, is a smooth path of symmetric Dirac type operators as above, and $P_{t} \in \operatorname{Gr}\left(A_{t}\right)$ is a smooth path, giving a smooth path of selfadjoint discrete operators $D_{P}(t)$.

Then

$$
\widetilde{\eta}\left(D_{P}(1)\right)-\widetilde{\eta}\left(D_{P}(0)\right)=\operatorname{SF}\left(D_{P}(t)\right)_{t \in[0,1]}+\frac{1}{2} \int_{0}^{1} \frac{d}{d t}\left(\eta\left(D_{P}(t)\right)\right) d t .
$$

Moreover, if the dimension of the kernel of $D_{P}(t)$ is independent of $t$, then the function $t \mapsto \widetilde{\eta}\left(D_{P}(t)\right)$ is smooth.

ProOF. We only sketch the proof, since this fact is well-known, at least when the $\eta$-function is regular at $s=0$, and the general case is proven by the same argument, because the pole of the $\eta$-function at $s=0$ is determined by the asymptotics of the spectrum, whereas the spectral flow depends only on the small eigenvalues.

Given $r \in[0,1]$, choose an $\varepsilon>0$ so that $\pm \varepsilon$ does not lie in the spectrum of $D_{P}(r)$. Applying standard results from perturbation theory 21] we infer that $\pm \varepsilon$ does not lie in the spectrum of $D_{P}(t)$ for $t$ close enough to $r$, say $t \in\left[t_{0}, t_{1}\right]$. Moreover the span of those eigenvectors of $D_{P}(t)$ whose eigenvalues lie in $(-\varepsilon, \varepsilon)$ varies continuously for $t \in\left[t_{0}, t_{1}\right]$.

Thus we can write $\eta\left(D_{P}(t) ; s\right)$ for $t \in\left[t_{0}, t_{1}\right]$ and $\operatorname{Re}(s)>>0$ as a sum

$$
\begin{aligned}
\eta\left(D_{P}(t) ; s\right) & =\sum_{\lambda \in \operatorname{spec} D_{P}(t), 0<|\lambda|<\varepsilon} \operatorname{sign}(\lambda)|\lambda|^{-s}+\sum_{\lambda \in \operatorname{spec}} \operatorname{sign}(\lambda)|\lambda|^{-s}(t),|\lambda|>\varepsilon \\
& =\eta_{<\varepsilon}\left(D_{P}(t) ; s\right)+\eta_{>\varepsilon}\left(D_{P}(t) ; s\right) .
\end{aligned}
$$


The sum $\eta_{<\varepsilon}\left(D_{P}(t) ; s\right)$ is finite, and so its analytic continuation to $s=0$ is integer valued:

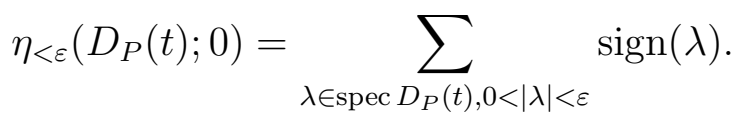

Thus

$$
\begin{aligned}
& \eta_{<\varepsilon}\left(D_{P}\left(t_{1}\right) ; 0\right)-\eta_{<\varepsilon}\left(D_{P}\left(t_{0}\right) ; 0\right) \\
& \quad=2 \operatorname{SF}\left(D_{P}(t)\right)_{t \in\left[t_{0}, t_{1}\right]}+\operatorname{dim} \operatorname{ker} D_{P}\left(t_{0}\right)-\operatorname{dim} \operatorname{ker} D_{P}\left(t_{1}\right) .
\end{aligned}
$$

Notice that this equation depends on our choice of convention for defining the spectral flow. The function $\eta_{>\varepsilon}\left(D_{P}(t) ; s\right)=\eta\left(D_{P}(t) ; s\right)-\eta_{<\varepsilon}\left(D_{P}(t) ; s\right)$ varies smoothly in $t \in$ $\left[t_{0}, t_{1}\right]$ since we have subtracted the eigenvalues that cross zero, and since no eigenvalues equal $\pm \varepsilon$ in this interval. If we define $\eta_{>\varepsilon}\left(D_{P}(t)\right)$ similarly to Definition 3.2 then $\eta_{>\varepsilon}\left(D_{P}(t)\right)$ is smooth and

$$
\eta_{>\varepsilon}\left(D_{P}(t)\right) \equiv \eta\left(D_{P}(t)\right) \bmod \mathbb{Z} .
$$

Therefore, using (3.4) we obtain

$$
\begin{aligned}
\int_{t_{0}}^{t_{1}} \frac{d}{d t}( & \left.\eta\left(D_{P}(t)\right)\right) d t \\
= & \int_{t_{0}}^{t_{1}} \frac{d}{d t}\left(\eta_{>\varepsilon}\left(D_{P}(t)\right)\right) d t \\
= & \eta_{>\varepsilon}\left(D_{P}\left(t_{1}\right)\right)-\eta_{>\varepsilon}\left(D_{P}\left(t_{0}\right)\right) \\
= & \eta\left(D_{P}\left(t_{1}\right)\right)-\eta_{<\varepsilon}\left(D_{P}\left(t_{1}\right)\right)-\eta\left(D_{P}\left(t_{0}\right)\right)+\eta_{<\varepsilon}\left(D_{P}\left(t_{0}\right)\right) \\
= & \eta\left(D_{P}\left(t_{1}\right)\right)-\eta\left(D_{P}\left(t_{0}\right)\right)-2 \mathrm{SF}\left(D_{P}(t)\right)_{t \in\left[t_{0}, t_{1}\right]} \\
& +\operatorname{dim} \operatorname{ker} D_{P}\left(t_{1}\right)-\operatorname{dim} \operatorname{ker} D_{P}\left(t_{0}\right) .
\end{aligned}
$$

Dividing by 2 proves the lemma over the interval $\left[t_{0}, t_{1}\right]$. The general case is obtained by covering the interval $[0,1]$ by small subintervals and adding the results.

For the last assertion, notice that if the dimension of $\operatorname{ker} D_{P}(t)$ is independent of $t$, then $\operatorname{SF}\left(D_{P}(t)\right)_{t \in[0, s]}=0$ for all $s \in[0,1]$.

\section{The Scott-Wojciechowski theorem}

The theorem of Scott and Wojciechowski [30 identifies the regularized $\zeta$-determinant of a boundary-value problem for a Dirac operator with a Fredholm determinant of the associated boundary projection. In this section we summarize and slightly extend that part of their result which we need, in the language of this article. Briefly, their theorem shows that the reduction $\bmod \mathbb{Z}$ of the $\eta$-invariant of $D_{P}$ for $P \in \operatorname{Gr}_{\infty}(A)$ and the Fredholm determinant of the unitary map $\Phi(P)$ which corresponds to $P$ via (2.10) agree up to a constant independent of $P$. The important consequence for this article is that the $\bmod \mathbb{Z}$ reduction of the $\eta$-invariant for a manifold with boundary depends only on the boundary data and the Calderón projector.

In this section $D$ denotes a fixed Dirac type operator on a manifold $X$ with boundary $\partial X$ and $A$ denotes its tangential operator. 
Before stating the Scott-Wojciechowski theorem, let us briefly recall the $\zeta-$ regularized determinant. Let $P \in \operatorname{Gr}_{\infty}(A)$. Then $\zeta\left(D_{P} ; s\right)$ is regular at $s=0$ and one puts

$$
\operatorname{det}_{\zeta} D_{P}:= \begin{cases}\exp \left(-\zeta^{\prime}\left(D_{P} ; 0\right)\right), & 0 \notin \operatorname{spec} D_{P} \\ 0, & 0 \in \operatorname{spec} D_{P}\end{cases}
$$

In view of (3.1) and Theorem 3.1 a straightforward calculation shows for $D_{P}$ invertible

$$
\operatorname{det}_{\zeta} D_{P}=\exp \left(i \frac{\pi}{2}\left(\zeta\left(D_{P}^{2} ; 0\right)-\eta\left(D_{P}\right)\right)-\frac{1}{2} \zeta^{\prime}\left(D_{P}^{2} ; 0\right)\right)
$$

We emphasize that the regularity of $\eta\left(D_{P} ; s\right)$ and $\zeta\left(D_{P} ; s\right)$ at $s=0$ is essential for (4.2) to hold. (4.2) implies that in general $\left(\operatorname{det}_{\zeta} D\right)^{2} \neq \operatorname{det}_{\zeta}\left(D^{2}\right)$. Note that Fredholm determinants are multiplicative, i.e. if $S, T$ are operators of determinant class in a

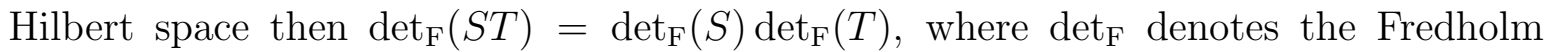
determinant.

With these preparations, the Scott-Wojciechowski theorem reads as follows.

Theorem 4.1. Let $P \in \operatorname{Gr}_{\infty}(A)$. Then

$$
\operatorname{det}_{\zeta}\left(D_{P}\right)=\operatorname{det}_{\zeta}\left(D_{P_{X}}\right) \operatorname{det}_{\mathrm{F}}\left(\frac{I+\Phi\left(P_{X}\right) \Phi(P)^{*}}{2}\right) .
$$

This result was proved for $M$ odd-dimensional in [30, Thms. 0.1, 1.4]. An alternative proof which applies to all dimensions and to slightly more general operators will be presented in 23].

In view of (4.2) the Scott-Wojciechowski theorem can be applied to express the dependence of $\widetilde{\eta}\left(D_{P}\right)$ on $P$ in terms of Fredholm determinants.

Let $P, Q \in \operatorname{Gr}_{\infty}(A)$. Since $\Phi(Q) \Phi(P)^{*}-I$ is a smoothing operator, it is of trace class and hence

$$
\frac{I+\Phi(Q) \Phi(P)^{*}}{2}=I+\frac{\Phi(Q) \Phi(P)^{*}-I}{2}
$$

is of determinant class. In particular, $\frac{I+\Phi\left(P_{X}\right) \Phi(P)^{*}}{2}$ is of determinant class and thus the right hand side in (4.3) is well-defined.

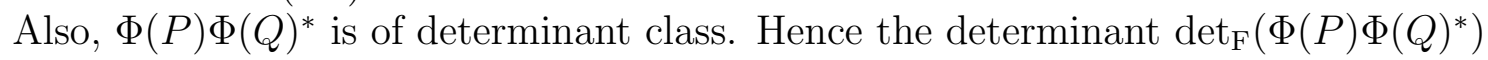
is defined and lies in $U(1)$ since $\Phi(P) \Phi(Q)^{*}$ is unitary.

Theorem 4.2. Let $P, Q \in \operatorname{Gr}_{\infty}(A)$. Then

$$
e^{2 \pi i\left(\widetilde{\eta}\left(D_{P}\right)-\widetilde{\eta}\left(D_{Q}\right)\right)}=\operatorname{det}_{\mathrm{F}}\left(\Phi(P) \Phi(Q)^{*}\right) .
$$

Proof. Assume first that $P$ is the Calderón projector $P_{X}$ and that the pair $\left(P_{X}, Q\right)$ is invertible. By Lemma 2.3 this means that $D_{P_{X}}$ and $D_{Q}$ are invertible. Putting Theorem 4.1 and (4.2) together and taking into account that $\zeta\left(D_{P}^{2} ; 0\right)$ is independent of $P$ (Theorem 3.1), we obtain

$$
e^{i \frac{\pi}{2}\left(\eta\left(D_{P_{X}}\right)-\eta\left(D_{Q}\right)\right)} e^{\frac{1}{2}\left(\zeta^{\prime}\left(D_{P_{X}}^{2} ; 0\right)-\zeta^{\prime}\left(D_{Q}^{2} ; 0\right)\right)}=\frac{\operatorname{det}_{\zeta}\left(D_{Q}\right)}{\operatorname{det}_{\zeta}\left(D_{P_{X}}\right)}=\operatorname{det}_{\mathrm{F}}\left(\frac{I+\Phi\left(P_{X}\right) \Phi(Q)^{*}}{2}\right),
$$


and thus

$$
\frac{\operatorname{det}_{\mathrm{F}}\left(\frac{I+\Phi\left(P_{X}\right) \Phi(Q)^{*}}{2}\right)}{\left|\operatorname{det}_{\mathrm{F}}\left(\frac{I+\Phi\left(P_{X}\right) \Phi(Q)^{*}}{2}\right)\right|}=e^{i \frac{\pi}{2}\left(\eta\left(D_{P_{X}}\right)-\eta\left(D_{Q}\right)\right)} .
$$

Since $\Phi\left(P_{X}\right) \Phi(Q)^{*}-I$ is of trace class we may choose a self-adjoint trace class operator $H$ such that $e^{i H}=\Phi\left(P_{X}\right) \Phi(Q)^{*}$. Then

$$
\begin{aligned}
\operatorname{det}_{\mathrm{F}}\left(\frac{I+\Phi\left(P_{X}\right) \Phi(Q)^{*}}{2}\right)^{2} & =\operatorname{det}_{\mathrm{F}}\left(\frac{I+e^{i H}}{2}\right)^{2} \\
& =\operatorname{det}_{\mathrm{F}}\left(e^{i H} \cosh ^{2}(H / 2)\right) \\
& =\operatorname{det}_{\mathrm{F}}\left(\Phi\left(P_{X}\right) \Phi(Q)^{*}\right) \operatorname{det}_{\mathrm{F}}\left(\cosh ^{2}(H / 2)\right),
\end{aligned}
$$

where we have used the multiplicativity of the Fredholm determinant in the last line. Consequently

$$
\frac{\operatorname{det}_{\mathrm{F}}\left(\frac{I+\Phi\left(P_{X}\right) \Phi(Q)^{*}}{2}\right)^{2}}{\left|\operatorname{det}_{\mathrm{F}}\left(\frac{I+\Phi\left(P_{X}\right) \Phi(Q)^{*}}{2}\right)\right|^{2}}=\operatorname{det}_{\mathrm{F}}\left(\Phi\left(P_{X}\right) \Phi(Q)^{*}\right) .
$$

Putting together (4.7) and (4.9) we obtain (4.5) for $P=P_{X}$ and $Q \in \operatorname{Gr}_{\infty}(A)$ such that $\left(P_{X}, Q\right)$ is an invertible pair. However, since both sides of (4.5) depend continuously on $Q$, (4.5) remains valid for all $Q \in \operatorname{Gr}_{\infty}(A)$. Finally, if $P, Q \in \operatorname{Gr}_{\infty}(A)$ are arbitrary then

$$
\begin{aligned}
e^{2 \pi i\left(\widetilde{\eta}\left(D_{P}\right)-\widetilde{\eta}\left(D_{Q}\right)\right)} & =e^{2 \pi i\left(\widetilde{\eta}\left(D_{P}\right)-\widetilde{\eta}\left(D_{P_{X}}\right)\right)} e^{2 \pi i\left(\widetilde{\eta}\left(D_{P_{X}}\right)-\widetilde{\eta}\left(D_{Q}\right)\right)} \\
& =\operatorname{det}_{\mathrm{F}}\left(\Phi(P) \Phi\left(P_{X}\right)^{*}\right) \operatorname{det}_{\mathrm{F}}\left(\Phi\left(P_{X}\right) \Phi(Q)^{*}\right) \\
& =\operatorname{det}_{\mathrm{F}}\left(\Phi(P) \Phi(Q)^{*}\right) .
\end{aligned}
$$

We will use the following convenient form of the Scott-Wojciechowski theorem. We consider the reals $\mathbb{R}$ as the universal cover of $U(1)$ via the map $r \mapsto e^{2 \pi i r}$.

Corollary 4.3. Let $P_{t}, t \in[0,1]$, be a smooth path in $\operatorname{Gr}_{\infty}(A)$. Then the map

$$
s \mapsto \frac{1}{2} \int_{0}^{s} \frac{d}{d t}\left(\eta\left(D_{P_{t}}\right)\right) d t
$$

is the unique lift to $\mathbb{R}$ of the map $[0,1] \rightarrow U(1)$

$$
s \mapsto \operatorname{det}_{\mathrm{F}}\left(\Phi\left(P_{s}\right) \Phi\left(P_{0}\right)^{*}\right) .
$$

In preparation for the next theorem, suppose that $P \in \operatorname{Gr}_{\infty}(A)$. From Lemma 2.3 we know that $D_{P}$ is invertible if and only if ker $P_{X} \cap \gamma($ ker $P)=0$ where $P_{X}$ denotes the Calderón projector; by Lemma 2.6 this happens if and only if $-1 \notin \operatorname{spec}\left(\Phi(P) \Phi\left(P_{X}\right)^{*}\right)$. In fact, the kernel of $D_{P}$ is canonically isomorphic to $\operatorname{ker}\left(I+\Phi(P) \Phi\left(P_{X}\right)^{*}\right)$.

Using the functional calculus we can define the operator $\log \left(\Phi(P) \Phi\left(P_{X}\right)^{*}\right)$. The choice of the branch of log will be essential in what follows. We define log: $\mathbb{C} \backslash\{0\} \rightarrow \mathbb{C}$ as follows

$$
\log \left(r e^{i t}\right)=\ln r+i t, \quad r>0,-\pi<t \leq \pi
$$


Since $-1 \notin \operatorname{spec}_{\text {ess }}\left(\Phi(P) \Phi\left(P_{X}\right)^{*}\right), \quad-1$ is an isolated point in the spectrum of $\Phi(P) \Phi\left(P_{X}\right)^{*}$ and thus we can choose a holomorphic branch of the logarithm which coincides on $\operatorname{spec}\left(\Phi(P) \Phi\left(P_{X}\right)\right)^{*}$ with log defined in (4.11). The so defined $\log \left(\Phi(P) \Phi\left(P_{X}\right)^{*}\right)$ is of trace class and

$$
\operatorname{tr} \log \left(\Phi(P) \Phi\left(P_{X}\right)^{*}\right) \equiv \log \operatorname{det}_{\mathrm{F}}\left(\Phi(P) \Phi\left(P_{X}\right)^{*}\right) \bmod 2 \pi i \mathbb{Z}
$$

After these preparations we can improve Theorem 4.2 as follows.

THEOREM 4.4. Let $X$ be a compact manifold with boundary and let $D$ be a Dirac type operator such that in a collar $\partial X \times[0, \varepsilon)$ of the boundary $D$ takes the form $D=$ $\gamma\left(\frac{d}{d x}+A\right)$ with $A, \gamma$ as in (2.1). Let $\Phi$ be the map defined in (2.7). Then for $P \in \operatorname{Gr}_{\infty}(A)$ we have

$$
\widetilde{\eta}\left(D_{P}\right)-\widetilde{\eta}\left(D_{P_{X}}\right)=\frac{1}{2 \pi i} \operatorname{tr} \log \left(\Phi(P) \Phi\left(P_{X}\right)^{*}\right)
$$

Proof. We assume first that $D_{P}$ is invertible. $D_{P_{X}}$ is invertible by Lemma 2.3. In view of Proposition 2.4 and Lemma 2.3 the space of those $P \in \operatorname{Gr}_{\infty}(A)$ so that $D_{P}$ is invertible is path connected. Choose a smooth path $P_{t}$ in $\operatorname{Gr}_{\infty}(A)$ starting at $P_{X}$ and ending at $P$ so that $D_{P_{t}}$ is invertible for all $t$.

The spectral flow of $D_{P_{t}}$ equals zero since the kernel is zero along the path and so Lemma 3.4 shows that $t \mapsto \widetilde{\eta}\left(D_{P_{t}}\right)$ is smooth. Hence

$$
t \mapsto \widetilde{\eta}\left(D_{P_{t}}\right)-\widetilde{\eta}\left(D_{P_{X}}\right)
$$

is smooth. Also, the map

$$
t \mapsto \frac{1}{2 \pi i} \operatorname{tr} \log \left(\Phi\left(P_{t}\right) \Phi\left(P_{X}\right)^{*}\right)
$$

is smooth since $-1 \notin \operatorname{spec}\left(\Phi\left(P_{t}\right) \Phi\left(P_{X}\right)^{*}\right)$ for all $t$ and hence log is holomorphic on $\operatorname{spec}\left(\Phi\left(P_{t}\right) \Phi\left(P_{X}\right)^{*}\right)$.

Theorem 4.2 states that the two smooth functions of (4.12) and (4.13) are the lifts to $\mathbb{R}$ of the same function to $U(1)=\mathbb{R} / \mathbb{Z}$, and they both start at 0 . Hence they coincide for all $t$.

Now let $P \in \operatorname{Gr}_{\infty}(A)$ be arbitrary. We may choose a path $\left(P_{t}\right)_{-\varepsilon \leq t \leq \varepsilon}$ in $\operatorname{Gr}_{\infty}(A)$ such that $\left(P_{t}, P_{X}\right)$ is invertible for $t \neq 0, P_{0}=P$, and such that at $t=0$ exactly $k=\operatorname{dim}$ ker $D_{P_{0}}$ eigenvalues of $\Phi\left(P_{t}\right) \Phi\left(P_{X}\right)^{*}$ cross -1 from the upper half plane to the lower half plane and no eigenvalues cross from the lower half plane to the upper half plane. To see this let $R$ be the orthogonal projection onto $\operatorname{ker}\left(I+\Phi(P) \Phi\left(P_{X}\right)^{*}\right)$. The projection $R$ is a pseudo-differential operator. Now put

$$
\Phi\left(P_{t}\right):=\left(e^{i(\pi+t)} R \oplus(I-R) \Phi(P) \Phi\left(P_{X}\right)^{*}\right) \Phi\left(P_{X}\right) .
$$

By our choice of log we then have

$$
\frac{1}{2 \pi i} \operatorname{tr} \log \left(\Phi\left(P_{0}\right) \Phi\left(P_{X}\right)^{*}\right)=\lim _{t \rightarrow 0-} \frac{1}{2 \pi i} \operatorname{tr} \log \left(\Phi\left(P_{t}\right) \Phi\left(P_{X}\right)^{*}\right) .
$$

Moreover, for $t \neq 0$ we have from the first part of this proof

$$
\widetilde{\eta}\left(D_{P_{t}}\right)-\widetilde{\eta}\left(D_{P_{X}}\right)=\frac{1}{2 \pi i} \operatorname{tr} \log \left(\Phi\left(P_{t}\right) \Phi\left(P_{X}\right)^{*}\right) .
$$


From Lemma 3.4, (4.14) and (4.16) one infers $\mathrm{SF}\left(D_{P_{t}}\right)_{-\varepsilon \leq t \leq \varepsilon}=-k$ and since $\operatorname{dim} \operatorname{ker} D_{P_{0}}=k$ at $t=0$ exactly $k$ eigenvalues of $D_{P_{t}}$ cross 0 from + to - and no eigenvalues cross from - to + . Hence

$$
\begin{aligned}
\widetilde{\eta}\left(D_{P_{0}}\right)-\widetilde{\eta}\left(D_{P_{X}}\right) & =\lim _{t \rightarrow 0-} \widetilde{\eta}\left(D_{P_{t}}\right)-\widetilde{\eta}\left(D_{P_{X}}\right) \\
& =\lim _{t \rightarrow 0-} \frac{1}{2 \pi i} \operatorname{tr} \log \left(\Phi\left(P_{t}\right) \Phi\left(P_{X}\right)^{*}\right) \\
& =\frac{1}{2 \pi i} \operatorname{tr} \log \left(\Phi\left(P_{0}\right) \Phi\left(P_{X}\right)^{*}\right),
\end{aligned}
$$

completing the proof.

\section{Splittings of manifolds and the $\eta$-invariant $\mathbf{I}$}

We consider now the gluing problem for the $\eta$-invariant. Suppose we are given a closed manifold $M$ containing a separating hypersurface $N \subset M$. We consider only Dirac operators $D$ on $M$ so that in a collar neighborhood $[-\varepsilon, \varepsilon] \times N$ of $N, D$ has the form $D=\gamma\left(\frac{d}{d x}+A\right)$ as in (2.1).

Let $M^{\text {cut }}$ denote the compact manifold with boundary obtained by cutting $M$ along $N$. Thus $M^{\text {cut }}$ is the disjoint union of two submanifolds $M^{+}$and $M^{-}$, with $\partial M^{+}$ and $\partial M^{-}$canonically identified with $N$. To apply the results of the previous section, we reparameterize the collar of $M^{-}$as $\partial M^{-} \times[0, \varepsilon]$ with $x=0$ corresponding to the boundary. See the following figure.

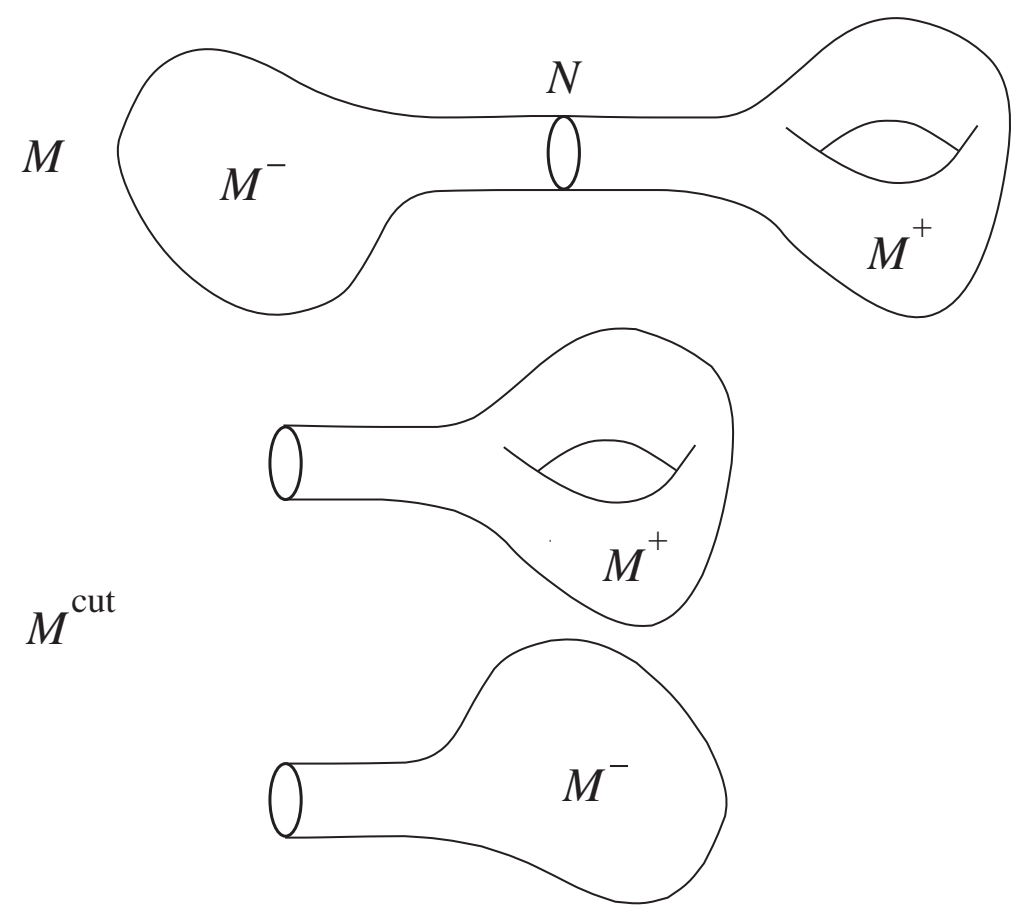

The manifolds $M$ and $M^{\text {cut }}$ 
The $H_{1}$-Sections of a bundle $E$ over $M$ correspond to sections $f \in H_{1}\left(E_{\mid M^{\text {cut }}}\right)$ over $M^{\text {cut }}$ so that $f_{\mid \partial M^{+}}=f_{\mid \partial M^{-}}$with respect to the canonical identifications $\partial M^{ \pm}=$ $N$. More precisely, the restriction of the section $f$ to the boundary of $M^{\text {cut }}$ lies in $H_{1 / 2}\left(E_{\mid \partial M^{\text {cut }}}\right) \subset L^{2}\left(E_{\mid \partial M^{\text {cut }}}\right)$. The identification of $\partial M^{\text {cut }}$ with two copies of $N$ gives a canonical decomposition

$$
L^{2}\left(E_{\mid \partial M^{\text {cut }}}\right)=L^{2}\left(E_{\mid N}\right) \oplus L^{2}\left(E_{\mid N}\right)
$$

where the first factor corresponds to $\partial M^{+}$and the second to $\partial M^{-}$. The restriction of a section $f$ over $M^{\text {cut }}$ to the boundary can thus be written as $\left(f_{+}, f_{-}\right)$, and the sections over $M$ correspond exactly to those $f$ so that $f_{+}=f_{-}$.

On the collar of $M^{\text {cut }}$, The operator $D$ takes the form

$$
D=\left(\begin{array}{cc}
\gamma & 0 \\
0 & -\gamma
\end{array}\right)\left(\frac{d}{d x}+\left(\begin{array}{cc}
A & 0 \\
0 & -A
\end{array}\right)\right)=: \widetilde{\gamma}\left(\frac{d}{d x}+\widetilde{A}\right)
$$

with respect to the decomposition (5.1) (this is because of the change of parameterization of the collar of $M^{-}$).

Note that the (closed) diagonal subspace

$$
\Delta=\left\{(f, f) \mid f \in L^{2}\left(E_{\mid N}\right)\right\} \subset L^{2}\left(E_{\mid N}\right) \oplus L^{2}\left(E_{\mid N}\right)
$$

is Lagrangian. In fact:

1. $\Delta$ is orthogonal to $\widetilde{\gamma}(\Delta)$ since

$$
\langle(f, f), \widetilde{\gamma}(g, g)\rangle=\langle f, \gamma g\rangle+\langle f,-\gamma g\rangle=0 .
$$

2. $\Delta+\widetilde{\gamma}(\Delta)=L^{2}\left(E_{\mid N}\right) \oplus L^{2}\left(E_{\mid N}\right)$ since

$$
(f, g)=\frac{1}{2}((f+g, f+g)+\widetilde{\gamma}(-\gamma f+\gamma g,-\gamma f+\gamma g)) .
$$

The orthogonal projection to $\Delta^{\perp}$ will be denoted by $P_{\Delta}$. It is called the continuous transmission projection. By construction an $H_{1}$-section $f$ over $M^{\text {cut }}$ defines an $H_{1}-$ section over $M$ if and only if the restriction $\left(f_{+}, f_{-}\right)$of $f$ to the boundary satisfies $P_{\Delta}\left(f_{+}, f_{-}\right)=0$. Note that $P_{\Delta} \in \operatorname{Gr}(\widetilde{A})$ since $D_{P_{\Delta}}$ is canonically identified with the (Fredholm) operator $D$ acting on the closed manifold $M$.

With respect to the decomposition (5.1) the operator $P_{\Delta}$ takes the form

$$
P_{\Delta}=\frac{1}{2}\left(\begin{array}{rr}
1 & -1 \\
-1 & 1
\end{array}\right)
$$

In a strict sense, the projection $P_{\Delta}$ is not in $\operatorname{Gr}(\widetilde{A})$ since it does not act as a pseudodifferential operator on $E_{\mid \partial M^{\text {cut }}}$. Namely, since $(5.3)$ contains off-diagonal terms the two copies of of $N \subset \partial M^{\text {cut }}$ interact and hence $P_{\Delta}$ is a Fourier integral operator. However, $P_{\Delta}$ is pseudo-differential on the bundle $E_{\mid N} \oplus E_{\mid N}$ over $N$. This is only a mild generalization of the situation of Section 2 and we refrain from formalizing it. From now on $\operatorname{Gr}(\widetilde{A})$ is to be understood as the set of those pseudo-differential operators on the bundle $E_{\mid N} \oplus E_{\mid N}$ over $N$ which satisfy (2), (3), (4) of Definition 2.1. It is fairly clear that the results of the previous sections also apply to this situation. 
There is a natural map

$$
\operatorname{Gr}(A) \times \operatorname{Gr}(-A) \longrightarrow \operatorname{Gr}(\widetilde{A}), \quad(P, Q) \mapsto\left(\begin{array}{cc}
P & 0 \\
0 & Q
\end{array}\right)
$$

with respect to the decomposition (5.1). In particular the Calderón projector for $M^{\text {cut }}$ takes the form

$$
P_{M^{\mathrm{cut}}}=\left(\begin{array}{cc}
P_{M^{+}} & 0 \\
0 & P_{M^{-}}
\end{array}\right)
$$

WARning. 1. There are two different decompositions of $L^{2}\left(E_{\mid \partial M^{\text {cut }}}\right)$, one coming from the $\pm i$ eigenspace decomposition of $\widetilde{\gamma}(2.5)$, and the second from the decomposition $\partial M^{\text {cut }}=N \coprod N(5.1)$. This leads to two different matrix representations of $P \in \operatorname{Gr}(\widetilde{A})$. These two decompositions are compatible since $\widetilde{\gamma}=\gamma \oplus(-\gamma)$, and so in fact one can write

$$
L^{2}\left(E_{\mid \partial M^{\mathrm{cut}}}\right)=\left(\mathscr{E}_{i} \oplus \mathscr{E}_{-i}\right) \oplus\left(\mathscr{E}_{-i} \oplus \mathscr{E}_{i}\right) .
$$

2. Although $P_{\Delta} \in \operatorname{Gr}(\widetilde{A})$, it is not in $\operatorname{Gr}_{\infty}(\widetilde{A})$. This fact causes technical difficulties.

3. It follows from (5.2) that if one parameterizes the collar of $M^{-}$as $\partial M^{-} \times[0, \varepsilon)$ then $\gamma$ is replaced by $-\gamma$ and $A$ is replaced by $-A$. This in particular means that the natural symplectic structure on $L^{2}\left(E_{\mid \partial M^{-}}\right)$is induced by $-\gamma$. Sometimes it will be crucial to distinguish between the map $\Phi_{\gamma}$ and the map $\Phi_{-\gamma}$ (cf. (2.7)). The relation between the two is

$$
\Phi_{-\gamma}(P)=-\Phi_{\gamma}(I-P)^{*}, \quad P \in \operatorname{Gr}(A)
$$

LEMma 5.1. Let $D$ be a Dirac operator on $M^{\text {cut }}$ and suppose that $P_{t}, 0 \leq t \leq 1$, is a continuous path in $\operatorname{Gr}(A)$ and $Q_{t}, 0 \leq t \leq 1$, is a continuous path in $\operatorname{Gr}(-A)$. Let

$$
B_{t}=\left(\begin{array}{cc}
P_{t} & 0 \\
0 & Q_{t}
\end{array}\right)
$$

be the corresponding path in $\operatorname{Gr}(\widetilde{A})$. Then

$$
\mathrm{SF}\left(D_{B_{t}}, M^{\text {cut }}\right)_{t \in[0,1]}=\mathrm{SF}\left(D_{P_{t}}, M^{+}\right)_{t \in[0,1]}+\mathrm{SF}\left(D_{Q_{t}}, M^{-}\right)_{t \in[0,1]} .
$$

Proof. This follows from the fact that

$$
L^{2}\left(E, M^{\text {cut }}\right)=L^{2}\left(E, M^{+}\right) \oplus L^{2}\left(E, M^{-}\right),
$$

and $D, B_{t}$ preserve this splitting. Hence, $D_{B_{t}}=D_{P_{t}}^{M^{+}} \oplus D_{Q_{t}}^{M^{-}}$. Note that the splitting (5.7) induces the splitting (5.1) by restricting to the boundary.

Notice that $P \in \operatorname{Gr}(A)$ if and only if $I-P \in \operatorname{Gr}(-A)$. Therefore, a particularly symmetric family of boundary conditions for $D$ acting on $M^{\text {cut }}$ is given by the image of $(P, I-P)$ under the map (5.4).

Corollary 4.3 to the Scott-Wojciechowski theorem implies the following lemma. 
Lemma 5.2. Let $D$ be a Dirac operator over $M$ and let $P_{0}, P_{1} \in \operatorname{Gr}_{\infty}(A)$. Choose a smooth path $P_{t} \in \operatorname{Gr}_{\infty}(A), 0 \leq t \leq 1$, from $P_{0}$ to $P_{1}$ and put

$$
Q_{t}:=\left(\begin{array}{cc}
P_{t} & 0 \\
0 & I-P_{t}
\end{array}\right) \in \operatorname{Gr}_{\infty}(\widetilde{A})
$$

Then

$$
\widetilde{\eta}\left(D_{Q_{1}}, M^{\text {cut }}\right)-\widetilde{\eta}\left(D_{Q_{0}}, M^{\text {cut }}\right)=\operatorname{SF}\left(D_{P_{t}}, M^{+}\right)_{t \in[0,1]}+\mathrm{SF}\left(D_{I-P_{t}}, M^{-}\right)_{t \in[0,1]} .
$$

In particular, the quantity $\mathrm{SF}\left(D_{P_{t}}, M^{+}\right)_{t \in[0,1]}+\mathrm{SF}\left(D_{I-P_{t}}, M^{-}\right)_{t \in[0,1]}$ is independent of the choice of the path $P_{t}$.

Proof. We know from Proposition 2.4 that $\operatorname{Gr}_{\infty}(A)$ is path connected. This assures the existence of a path $P_{t}$. Furthermore, notice that

$$
\widetilde{\eta}\left(D_{Q_{t}}, M^{\text {cut }}\right)=\widetilde{\eta}\left(D_{P_{t}}, M^{+}\right)+\widetilde{\eta}\left(D_{I-P_{t}}, M^{-}\right)
$$

since $D$ and $Q_{t}$ preserve the splitting of $L^{2}\left(E_{\mid M^{\text {cut }}}\right)=L^{2}\left(E_{\mid M^{+}}\right) \oplus L^{2}\left(E_{\mid M^{-}}\right)$.

Lemma 3.4 and Corollary 4.3 imply that

$$
\begin{aligned}
\widetilde{\eta}\left(D_{P_{1}}, M^{+}\right)-\widetilde{\eta}\left(D_{P_{0}}, M^{+}\right)-\operatorname{SF}\left(D_{P_{t}}, M^{+}\right)_{t \in[0,1]} \\
=\frac{1}{2} \int_{0}^{1} \frac{d}{d t}\left(\eta\left(D_{P_{t}}\right)\right) d t=\frac{1}{2 \pi i} \int_{0}^{1} \frac{d}{d t} \log \operatorname{det}_{\mathrm{F}}\left(\Phi\left(P_{t}\right) \Phi\left(P_{0}\right)^{*}\right) d t
\end{aligned}
$$

and

$$
\begin{aligned}
\widetilde{\eta}\left(D_{I-P_{1}},\right. & \left.M^{-}\right)-\widetilde{\eta}\left(D_{I-P_{0}}, M^{-}\right)-\mathrm{SF}\left(D_{I-P_{t}}, M^{-}\right)_{t \in[0,1]} \\
& =\frac{1}{2} \int_{0}^{1} \frac{d}{d t}\left(\eta\left(D_{I-P_{t}}\right)\right) d t=\frac{1}{2 \pi i} \int_{0}^{1} \frac{d}{d t} \log \operatorname{det}_{\mathrm{F}}\left(\Phi_{-\gamma}\left(I-P_{t}\right) \Phi_{-\gamma}\left(I-P_{0}\right)^{*}\right) d t .
\end{aligned}
$$

Note that in (5.9) $\Phi$ is taken with respect to $\gamma$ and in (5.10) $\Phi$ is taken with respect to $-\gamma$ (cf. 3. of the warning above). In view of (5.6) we find

$$
\operatorname{det}_{\mathrm{F}}\left(\Phi_{-\gamma}\left(I-P_{t}\right) \Phi_{-\gamma}\left(I-P_{0}\right)^{*}\right)=\operatorname{det}_{\mathrm{F}}\left(\Phi\left(P_{t}\right)^{*} \Phi\left(P_{0}\right)\right)=\overline{\operatorname{det}_{\mathrm{F}}\left(\Phi\left(P_{t}\right) \Phi\left(P_{0}\right)^{*}\right)},
$$

and consequently,

$$
\frac{d}{d t} \log \operatorname{det}_{\mathrm{F}}\left(\Phi_{-\gamma}\left(I-P_{t}\right) \Phi_{-\gamma}\left(I-P_{0}\right)^{*}\right)=-\frac{d}{d t} \log \operatorname{det}_{\mathrm{F}}\left(\Phi\left(P_{t}\right) \Phi\left(P_{0}\right)^{*}\right) .
$$

Adding (5.9) and (5.10) and using (5.8) gives the desired formula.

For any $P \in \operatorname{Gr}(A)$ a natural path connecting $P_{\Delta}$ and $\left(\begin{array}{cc}P & 0 \\ 0 & I-P\end{array}\right)$ is given by (cf. [7, Sec. 3])

$$
P(\theta, P):=\left(\begin{array}{cc}
\cos ^{2}(\theta) P+\sin ^{2}(\theta)(I-P) & -\cos (\theta) \sin (\theta) \\
-\cos (\theta) \sin (\theta) & \cos ^{2}(\theta)(I-P)+\sin ^{2}(\theta) P
\end{array}\right) .
$$


A straightforward calculation shows that $\xi=\left(\begin{array}{l}\xi_{+} \\ \xi_{-}\end{array}\right) \in \operatorname{ker} P(\theta, P)$ if and only if

$$
\begin{aligned}
\cos (\theta) P \xi_{+} & =\sin (\theta) P \xi_{-}, \\
\sin (\theta)(I-P) \xi_{+} & =\cos (\theta)(I-P) \xi_{-} .
\end{aligned}
$$

Lemma 5.3. Let $P \in \operatorname{Gr}(A)$. If $\cos (\theta) \neq 0$ then the map $P(\theta, P)$ lies in $\operatorname{Gr}(\widetilde{A})$. Furthermore

$$
P(0, P)=\left(\begin{array}{cc}
P & 0 \\
0 & I-P
\end{array}\right) \quad \text { and } \quad P\left(\frac{\pi}{4}, P\right)=P_{\Delta} .
$$

Proof. Fix a Lagrangian subspace $L \subset \operatorname{ker} A$ and let $P^{+}=P^{+}(L)$. The only part which is not straightforward is the claim that $\left(P(\theta, P), \widetilde{P}^{+}\right), \widetilde{P}^{+}:=\left(\begin{array}{cc}P^{+} & 0 \\ 0 & I-P^{+}\end{array}\right)$is a Fredholm pair. We use the following criterion (cf. [6, Remark 3.5]).

Two orthogonal projections $Q, R$ in a Hilbert space form a Fredholm pair (invertible pair) if and only if the operator $Q R Q+(I-Q)(I-R)(I-Q)$ is Fredholm (invertible).

One calculates

$$
\begin{aligned}
\widetilde{P}^{+} & P(\theta) \widetilde{P}^{+}+\left(I-\widetilde{P}^{+}\right)(I-P(\theta, P))\left(I-\widetilde{P}^{+}\right) \\
= & \left(\cos ^{2}(\theta)\left(P^{+} P P^{+}+\left(I-P^{+}\right)(I-P)\left(I-P^{+}\right)\right)+\right. \\
& \left.\sin ^{2}(\theta)\left(P^{+}(I-P) P^{+}+\left(I-P^{+}\right) P\left(I-P^{+}\right)\right)\right) \otimes\left(\begin{array}{ll}
I & 0 \\
0 & I
\end{array}\right) \\
\geq & \cos ^{2}(\theta)\left(P^{+} P P^{+}+\left(I-P^{+}\right)(I-P)\left(I-P^{+}\right)\right) \otimes\left(\begin{array}{ll}
I & 0 \\
0 & I
\end{array}\right) .
\end{aligned}
$$

Hence if $\cos (\theta) \neq 0$ then the pair $\left(P(\theta, P), \widetilde{P}^{+}\right)$is Fredholm (invertible) if the pair $\left(P, P^{+}\right)$is Fredholm (invertible).

We emphasize that even if $P \in \operatorname{Gr}_{\infty}(A)$ then $P(\theta, P) \notin \operatorname{Gr}_{\infty}(A)$ if $\sin (\theta) \neq 0$. The significance of the family $P(\theta, P)$ stems from the fact that $D_{P_{\Delta}}$ is naturally unitarily equivalent to $D$ acting on the closed manifold $M$.

We first note some consequences of the existence of the family $P(\theta, P)$ which do not make use of $\eta$-functions.

Proposition 5.4. Let $D$ be a Dirac operator over $M$ and let $P_{0}, P_{1} \in \operatorname{Gr}(A)$. Choose a smooth path $P_{t} \in \operatorname{Gr}(A), 0 \leq t \leq 1$, from $P_{0}$ to $P_{1}$ and put, as in Lemma 5.2 ,

$$
Q_{t}:=\left(\begin{array}{cc}
P_{t} & 0 \\
0 & I-P_{t}
\end{array}\right) \in \operatorname{Gr}_{\infty}(\widetilde{A})
$$

Then

$$
\mathrm{SF}\left(D_{P\left(\theta, P_{1}\right)}, M^{\text {cut }}\right)_{\theta \in\left[0, \frac{\pi}{4}\right]}-\mathrm{SF}\left(D_{P\left(\theta, P_{0}\right)}, M^{\text {cut }}\right)_{\theta \in\left[0, \frac{\pi}{4}\right]}+\mathrm{SF}\left(D_{Q_{t}}, M^{\text {cut }}\right)_{t \in[0,1]}=0 .
$$


Proof. Note again that in view of Proposition 2.4 the space $\operatorname{Gr}(A)$ is path connected. Using $P_{t}$ one obtains a map $H:\left[0, \frac{\pi}{4}\right] \times[0,1] \rightarrow \operatorname{Gr}(\widetilde{A})$ :

$$
H(\theta, t)=P\left(\theta, P_{t}\right) \text {. }
$$

Since $H\left(\frac{\pi}{4}, t\right)$ is the constant map at $P_{\Delta}$, one sees that the path

$$
\theta \mapsto H(\theta, 0)=P\left(\theta, P_{0}\right), \quad 0 \leq \theta \leq \frac{\pi}{4},
$$

is homotopic to the composite of the paths

$$
t \mapsto H(0, t)=Q_{t}, \quad 0 \leq t \leq 1,
$$

and

$$
\theta \mapsto H(\theta, 1)=P\left(\theta, P_{1}\right)
$$

The claim now follows from the homotopy invariance and additivity of the spectral flow.

Proposition 5.5. For the Calderón projectors $P_{M^{+}}$of $M^{+}$and $P_{M^{\text {cut }}}$ of $M^{\text {cut }}$, the space ker $P\left(\theta, P_{M^{+}}\right) \cap \operatorname{ker} \widetilde{\gamma}\left(P_{M^{\text {cut }}}\right)$ is canonically isomorphic to $\operatorname{im} P_{M^{+}} \cap \operatorname{im} P_{M^{-}}$. In particular, its dimension is independent of $\theta \in\left[0, \frac{\pi}{4}\right]$. Moreover,

$$
\mathrm{SF}\left(D_{P\left(\theta, P_{M^{+}}\right)}, M^{\text {cut }}\right)_{\theta \in\left[0, \frac{\pi}{4}\right]}=0 .
$$

Furthermore, if $P \in \operatorname{Gr}(A)$ and $P_{t}, 0 \leq t \leq 1$, is a smooth path in $\operatorname{Gr}(A)$ from $P$ to the Calderón projector $P_{M^{+}}$then

$$
\mathrm{SF}\left(D_{P(\theta, P)}, M^{\text {cut }}\right)_{\theta \in\left[0, \frac{\pi}{4}\right]}=\mathrm{SF}\left(D_{Q_{t}}, M^{\text {cut }}\right)_{t \in[0,1]},
$$

where $Q_{t}=P_{t} \oplus\left(I-P_{t}\right)$ as in (5.16).

Proof. By Lemma 2.3 the space $\operatorname{ker} P\left(\theta, P_{M^{+}}\right) \cap \operatorname{ker} \widetilde{\gamma}\left(P_{M^{\text {cut }}}\right)$ is isomorphic to ker $D_{P\left(\theta, P_{M^{+}}\right)}$. Hence, if we can show that $\operatorname{ker} P\left(\theta, P_{M^{+}}\right) \cap \operatorname{ker} \widetilde{\gamma}\left(P_{M^{\text {cut }}}\right)$ is independent of $\theta$, then $\operatorname{SF}\left(D_{P\left(\theta, P_{M^{+}}\right)}, M^{\text {cut }}\right)_{\theta \in\left[0, \frac{\pi}{4}\right]}=0$. means

Consider $\xi=\left(\begin{array}{l}\xi_{+} \\ \xi_{-}\end{array}\right) \in \operatorname{ker} P\left(\theta, P_{M^{+}}\right) \cap \operatorname{ker} \widetilde{\gamma}\left(P_{M^{\text {cut }}}\right)$. In view of (5.5) and (5.14) this

$$
\begin{aligned}
& \cos (\theta) P_{M^{+}} \xi_{+}=\sin (\theta) P_{M^{+}} \xi_{-}, \\
& 0=\sin (\theta)\left(I-P_{M^{+}}\right) \xi_{+}=\cos (\theta)\left(I-P_{M^{+}}\right) \xi_{-} .
\end{aligned}
$$

Since $\cos (\theta) \neq 0$ we infer $\xi_{-} \in \operatorname{im} P_{M^{+}} \cap \operatorname{im} P_{M^{-}}$.

Conversely, given $\xi_{-} \in \operatorname{im} P_{M^{+}} \cap \operatorname{im} P_{M^{-}}$put $\xi_{+}:=\tan (\theta) \xi_{-}$. Then (5.20) implies that $\left(\begin{array}{l}\xi_{+} \\ \xi_{-}\end{array}\right) \in \operatorname{ker} P\left(\theta, P_{M^{+}}\right) \cap \operatorname{ker} \widetilde{\gamma}\left(P_{M^{\text {cut }}}\right)$.

(5.19) is an immediate consequence of (5.18) and Proposition 5.4. 
Corollary 5.6. Let $M$ be a split manifold and let $D(t), a \leq t \leq b$, be a smooth path of Dirac type operators such that in a collar of the separating hypersurface we have $D(t)=\gamma\left(\frac{d}{d x}+A(t)\right)$. Let $P_{M^{+}}(t)$ be the corresponding family of Calderón projectors. Then

$$
\mathrm{SF}(D(t))_{t \in[a, b]}=\mathrm{SF}\left(D_{I-P_{M^{+}}(t)}(t), M^{-}\right)_{t \in[a, b]} \cdot
$$

Proof. We note that it was proved in 26] that $P_{M^{+}}(t)$ is smooth. Consider the two parameter family of operators on $M^{\text {cut }}$

$$
\left(D_{P\left(\theta, P_{M^{+}}(t)\right)}(t), M^{\text {cut }}\right)_{0 \leq \theta \leq \frac{\pi}{4}, a \leq t \leq b} .
$$

By Proposition 5.5 for fixed $t$ the dimension of the kernel of $D_{P\left(\theta, P_{M^{+}}(t)\right)}(t)$ is independent of $\theta$. By the homotopy invariance of the spectral flow this implies

$$
\mathrm{SF}\left(D_{P\left(0, P_{M^{+}}(t)\right)}(t), M^{\text {cut }}\right)_{t \in[a, b]}=\mathrm{SF}\left(D_{P\left(\frac{\pi}{4}, P_{M^{+}}(t)\right)}(t), M^{\text {cut }}\right)_{t \in[a, b]} .
$$

Since $P\left(\frac{\pi}{4}\right)=P_{\Delta}$ the right hand side equals $\mathrm{SF}(D(t))_{t \in[a, b]}$. The left hand side equals

$$
\mathrm{SF}\left(D_{P_{M^{+}}(t)}(t), M^{+}\right)_{t \in[a, b]}+\mathrm{SF}\left(D_{I-P_{M^{+}}(t)}, M^{-}\right)_{t \in[a, b]}
$$

and since $D_{P_{M}^{+}(t)}(t)$ is invertible its spectral flow vanishes and we reach the desired conclusion.

REMARK 5.7. We emphasize that we did not use $\eta$-invariants to prove Proposition 5.4, Proposition 5.5, and Corollary 5.6. The only ingredients of the proof are the family $P(\theta, P)$ and basic properties of the spectral flow.

We now return to the discussion of $\eta$-invariants. Since $D_{P_{\Delta}}$ is naturally unitarily equivalent to $D$ acting on the closed manifold $M$, we have for any $P \in \operatorname{Gr}(A)$

$$
\eta\left(D_{P\left(\frac{\pi}{4}, P\right)}, M^{\text {cut }}\right)=\eta\left(D_{P_{\Delta}}, M^{\text {cut }}\right)=\eta(D, M) .
$$

On the other hand, $D_{P(0, P)}$ is the direct sum of $D_{P}$ acting on $M^{+}$and $D_{I-P}$ acting on $M^{-}$. Therefore,

$$
\eta\left(D_{P(0, P)}, M^{\text {cut }}\right)=\eta\left(D_{P}, M^{+}\right)+\eta\left(D_{I-P}, M^{-}\right) .
$$

Hence, by Lemma 3.4 we have

$$
\begin{aligned}
\eta(D, M)= & \eta\left(D_{P}, M^{+}\right)+\eta\left(D_{I-P}, M^{-}\right) \\
& +\frac{1}{2} \int_{0}^{\frac{\pi}{4}} \frac{d}{d \theta} \eta\left(D_{P(\theta, P)}, M^{\text {cut }}\right) d t+\operatorname{SF}\left(D_{P(\theta, P)}\right)_{\theta \in\left[0, \frac{\pi}{4}\right]}
\end{aligned}
$$

Thus, in order to obtain a splitting theorem for the $\eta$-invariant one needs to understand the last two terms on the right hand side of (5.23). If $P$ is the Calderón projector of $M^{+}$or $M^{-}$then by Proposition 5.5 the spectral flow term vanishes.

Consider now the Atiyah-Patodi-Singer projection $P^{+}=P^{+}(L)$ of (2.3). The following theorem is the main result of the article [7] by J. Brüning and M. Lesch ([7, Theorem 3.9], see also (3.68) of loc.cit. with $T_{+}=-T_{-}^{*}$ determined by the choice of Lagrangian $L \subset \operatorname{ker} A$ ). 
TheOREM 5.8. Let $P^{+}=P^{+}(L)$ be the Atiyah-Patodi-Singer projection and let $P\left(\theta, P^{+}\right)_{\theta \in\left[0, \frac{\pi}{4}\right]}$ the deformation (5.13) to the continuous transmission projection. Then

$$
\frac{d}{d \theta}\left(\eta\left(D_{P\left(\theta, P^{+}\right)}, M^{\text {cut }}\right)=0 .\right.
$$

In view of (5.23) we conclude from Theorem 5.8 that

$$
\widetilde{\eta}(D, M)-\widetilde{\eta}\left(D_{P^{+}}, M^{+}\right)-\widetilde{\eta}\left(D_{I-P^{+}}, M^{-}\right)=\mathrm{SF}\left(D_{P\left(\theta, P^{+}\right)}, M^{\text {cut }}\right)_{\theta \in\left[0, \frac{\pi}{4}\right]} .
$$

Since the right hand side of (5.24) is an integer this formula implies the $\bmod \mathbb{Z}$ gluing formula for the $\eta$-invariant (see [7] for a discussion of the history of this result). Note that (5.24) is slightly weaker than Theorem 5.8.

Our strategy to obtain a useful splitting theorem for the $\eta$-invariant can now be explained. On the one hand (5.24) gives a complete splitting formula for the $\eta$-invariant with respect to Atiyah-Patodi-Singer boundary conditions, but it contains the (in general) uncomputable term $\operatorname{SF}\left(D_{P\left(\theta, P^{+}\right)}\right)_{\theta \in\left[0, \frac{\pi}{4}\right]}$. On the other hand if we were to replace $P^{+}$by the Calderón projector $P_{M^{+}}$, Proposition 5.5 shows that the corresponding spectral flow term vanishes. Thus Theorem 5.8 (or at least (5.24)) needs to be extended to a more general class of projections, including the Calderón projector. One possible strategy would be to generalize the arguments of [7] to more general projections. This might be manageable but technically tedious. Here, we will use a simpler approach which shows slightly less. Lemma 5.2 and Proposition 5.5 lead to a generalization of (5.24) to projections in $\mathrm{Gr}_{\infty}(A)$. This is less than a generalization of Theorem 5.8 since the variation of the $\eta$-invariant with respect to the path $P(\theta, P)$ might be non-zero.

Theorem 5.9. Let $D$ be a Dirac operator on $M$ and let $N \subset M$ split $M$ into $M^{+}$ and $M^{-}$. We assume that in a collar neighborhood $[-\varepsilon, \varepsilon] \times N$ of $N, D$ has the form $D=\gamma\left(\frac{d}{d x}+A\right)$ as in (2.1). Let $P \in \mathrm{Gr}_{\infty}(A)$ and let $P_{t}$ be a smooth path in $\mathrm{Gr}_{\infty}(A)$ from $P$ to the Calderón projector $P_{M^{+}}$. As in (5.16) put $Q_{t}:=P_{t} \oplus\left(I-P_{t}\right)$. Then

$$
\begin{aligned}
\widetilde{\eta}(D, M) & =\widetilde{\eta}\left(D_{P}, M^{+}\right)+\widetilde{\eta}\left(D_{I-P}, M^{-}\right)+\operatorname{SF}\left(D_{P(\theta, P)}, M^{\text {cut }}\right)_{\theta \in\left[0, \frac{\pi}{4}\right]} \\
& =\widetilde{\eta}\left(D_{P}, M^{+}\right)+\widetilde{\eta}\left(D_{I-P}, M^{-}\right)+\operatorname{SF}\left(D_{Q_{t}}, M^{\text {cut }}\right)_{t \in[0,1]} .
\end{aligned}
$$

In particular, if $P_{M^{+}}$is the Calderón projector for $M^{+}$then

$$
\widetilde{\eta}(D, M)=\widetilde{\eta}\left(D_{P_{M^{+}}}, M^{+}\right)+\widetilde{\eta}\left(D_{I-P_{M^{+}}}, M^{-}\right) .
$$

Proof. Fix a Lagrangian subspace $L \subset$ ker $A$ and choose a smooth path $R_{t} \in$ $\operatorname{Gr}_{\infty}(A)$ from $P$ to the Atiyah-Patodi-Singer projection $P^{+}=P^{+}(L)$. Set $\widetilde{R}_{t}:=$ $R_{t} \oplus\left(I-R_{t}\right)$. By Proposition 5.4 we have

$$
\mathrm{SF}\left(D_{P(\theta, P)}, M^{\text {cut }}\right)_{\theta \in\left[0, \frac{\pi}{4}\right]}=\mathrm{SF}\left(D_{P\left(\theta, P^{+}\right)}, M^{\text {cut }}\right)_{\theta \in\left[0, \frac{\pi}{4}\right]}+\mathrm{SF}\left(D_{\widetilde{R}_{t}}, M^{\text {cut }}\right)_{t \in[0,1]} .
$$


$\eta$-INVARIANT, MASLOV INDEX, AND SPECTRAL FLOW

Using Lemma 5.2 and (5.24) we obtain

$$
\begin{aligned}
& \widetilde{\eta}(D, M)-\widetilde{\eta}\left(D_{P}, M^{+}\right)-\widetilde{\eta}\left(D_{I-P}, M^{-}\right) \\
& =\widetilde{\eta}(D, M)-\widetilde{\eta}\left(D_{P^{+}}, M^{+}\right)-\widetilde{\eta}\left(D_{I-P^{+}}, M^{-}\right)+\widetilde{\eta}\left(D_{\widetilde{R}_{1}}, M^{\text {cut }}\right)-\widetilde{\eta}\left(D_{\widetilde{R}_{0}}, M^{\text {cut }}\right) \\
& =\operatorname{SF}\left(D_{P\left(\theta, P^{+}\right)}, M^{\text {cut }}\right)_{\theta \in\left[0, \frac{\pi}{4}\right]}+\operatorname{SF}\left(D_{\widetilde{R}_{t}}, M^{\text {cut }}\right)_{t \in[0,1]} \\
& =\operatorname{SF}\left(D_{P(\theta, P)}, M^{\text {cut }}\right)_{\theta \in\left[0, \frac{\pi}{4}\right]} .
\end{aligned}
$$

This proves the first line of (5.25). The second line of (5.25) and the last assertion follow from Proposition 5.5.

Notice that by symmetry the same argument also shows that

$$
\widetilde{\eta}(D, M)=\widetilde{\eta}\left(D_{I-P_{M^{-}}}, M^{+}\right)+\widetilde{\eta}\left(D_{P_{M^{-}}}, M^{-}\right) .
$$

Applying Theorem 4.4 allows us to extend Theorem 5.9 as follows.

TheOREM 5.10. Let $D$ be a Dirac operator on $M$ and let $N \subset M$ split $M$ into $M^{+}$ and $M^{-}$. Then for $P \in \operatorname{Gr}_{\infty}(A)$ and $Q \in \operatorname{Gr}_{\infty}(-A)$ we have, with $\Phi=\Phi_{\gamma}$,

$$
\begin{aligned}
\widetilde{\eta}(D, M)-\widetilde{\eta}\left(D_{P}, M^{+}\right)-\widetilde{\eta}\left(D_{Q}, M^{-}\right) \\
=-\frac{1}{2 \pi i} \operatorname{tr} \log \left(\Phi(P) \Phi\left(P_{M^{+}}\right)^{*}\right)-\frac{1}{2 \pi i} \operatorname{tr} \log \left(\Phi\left(P_{M^{-}}\right) \Phi(Q)^{*}\right) \\
\quad+\frac{1}{2 \pi i} \operatorname{tr} \log \left(\Phi\left(I-P_{M^{-}}\right) \Phi\left(P_{M^{+}}\right)^{*}\right) .
\end{aligned}
$$

In particular,

$$
\widetilde{\eta}(D, M)=\widetilde{\eta}\left(D_{P_{M^{+}}}, M^{+}\right)+\widetilde{\eta}\left(D_{P_{M^{-}}}, M^{-}\right)+\frac{1}{2 \pi i} \operatorname{tr} \log \left(\Phi\left(I-P_{M^{-}}\right) \Phi\left(P_{M^{+}}\right)^{*}\right) .
$$

Proof. It was remarked after (2.4) that $P_{M^{+}} \in \operatorname{Gr}_{\infty}(A)$ and $P_{M^{-}} \in \operatorname{Gr}_{\infty}(-A)$. Consequently, $I-P_{M^{-}} \in \operatorname{Gr}_{\infty}(A)$ and hence $I-P_{M^{-}}-P_{M^{+}}$is trace class.

Theorem 5.9 implies that $\widetilde{\eta}(D, M)-\widetilde{\eta}\left(D_{P}, M^{+}\right)-\widetilde{\eta}\left(D_{Q}, M^{-}\right)$is equal to

$$
\begin{aligned}
& -\left(\widetilde{\eta}\left(D_{P}, M^{+}\right)-\widetilde{\eta}\left(D_{P_{M^{+}}}, M^{+}\right)\right)-\left(\widetilde{\eta}\left(D_{Q}, M^{-}\right)-\widetilde{\eta}\left(D_{P_{M^{-}}}, M^{-}\right)\right) \\
& -\left(\widetilde{\eta}\left(D_{P_{M^{-}}}, M^{-}\right)-\widetilde{\eta}\left(D_{I-P_{M^{+}}}, M^{-}\right)\right) .
\end{aligned}
$$

Applying Theorem 4.4 to the three summands in (5.28) and taking (5.6) into account gives the assertion.

The formula (5.27) expresses the $\widetilde{\eta}$-invariant of $D$ on $M$ in terms of two $\widetilde{\eta}$-invariants intrinsic to the two pieces $M^{+}$and $M^{-}$of the decomposition and an "interaction" term.

\section{Maslov index and winding number}

In this section we compile the necessary material about the Maslov index and the winding number. One important comment is that in constructing the various invariants (winding number, Maslov index, triple index, spectral flow, and the branch of the logarithm) conventions must be chosen to set signs and to handle degenerate cases. In particular, care must be taken to ensure that the different possible conventions are chosen compatibly. Thus, although some of the material we present here is a generalization of ideas which appear in the literature, the subtleties arising in organizing the conventions compatibly and extending the constructions from the finite-dimensional to the infinite-dimensional context require the careful exposition we present. 
6.1. Winding number. Let $H$ be a complex Hilbert space and denote by $\mathscr{U}(H)$ the group of unitary operators on $H$. Similarly to (2.8), (2.9) we introduce the following subspaces:

$$
\begin{aligned}
\mathscr{U}_{*}(H) & :=\{U \in \mathscr{U}(H) \mid-1 \notin \operatorname{spec} U\}, \\
\mathscr{U}_{\text {Fred }}(H) & :=\left\{U \in \mathscr{U}(H) \mid-1 \notin \operatorname{spec}_{\text {ess }} U\right\}, \\
\mathscr{U}_{\mathscr{K}}(H) & :=\{U \in \mathscr{U}(H) \mid U-I \text { is compact }\}, \\
\mathscr{U}_{\mathrm{tr}}(H) & :=\{U \in \mathscr{U}(H) \mid U-I \text { is trace class }\} .
\end{aligned}
$$

The spaces $\mathscr{U}_{*}(H)$ and $\mathscr{U}_{\text {Fred }}(H)$ are not groups. It is well-known that the inclusion $\mathscr{U}_{\text {tr }}(H) \hookrightarrow \mathscr{U}_{\mathscr{K}}(H)$ is a homotopy equivalence and that $\mathscr{U}_{\mathscr{K}}(H)$ is homotopy equivalent to the infinite unitary group $\mathscr{U}(\infty)=\lim _{n \rightarrow \infty} \mathscr{U}(n)$. Therefore, one has by Bott periodicity

$$
\begin{aligned}
\pi_{2 k}\left(\mathscr{U}_{\mathscr{K}}(H)\right) & =\pi_{2 k}\left(\mathscr{U}_{\mathrm{tr}}(H)\right)=0, \\
\pi_{2 k+1}\left(\mathscr{U}_{\mathscr{K}}(H)\right) & =\pi_{2 k+1}\left(\mathscr{U}_{\mathrm{tr}}(H)\right) \simeq \mathbb{Z}, \quad k=0,1,2, \ldots
\end{aligned}
$$

Furthermore, the isomorphism $\pi_{1}\left(\mathscr{U}_{\mathrm{tr}}(H)\right) \rightarrow \mathbb{Z}$ is given by the winding number. I.e. if $f:[0,1] \rightarrow \mathscr{U}_{\mathrm{tr}}(H)$ is a closed $C^{1}$-path then

$$
\operatorname{wind}(f):=\frac{1}{2 \pi i} \int_{0}^{1} \operatorname{tr}\left(f(t)^{-1} f^{\prime}(t)\right) d t .
$$

\section{LEMMA 6.1.}

1. The inclusion $\mathscr{U}_{\mathscr{K}}(H) \rightarrow \mathscr{U}_{\text {Fred }}(H)$ is a weak homotopy equivalence,

2. For any $U \in \mathscr{U}_{\text {Fred }}(H)$ there exists a smooth path $f:[0,1] \rightarrow \mathscr{U}_{\text {Fred }}(H)$ such that $f(0)-I$ is of finite rank, $f(1)=U$, and such that $\operatorname{dim} \operatorname{ker}(f(t)+I)$ is independent of $t$.

Proof. 1. Let $\mathscr{Q}(H):=\mathscr{B}(H) / \mathscr{K}(H)$ be the Calkin algebra. Then the quotient $\operatorname{map} \sigma: \mathscr{B}(H) \rightarrow \mathscr{Q}(H)$ sends $\mathscr{U}_{\text {Fred }}(H)$ onto $\{u \in \mathscr{Q}(H) \mid-1 \notin \operatorname{spec} u\}=: \mathscr{U}_{*} \mathscr{Q}(H)$. Moreover, $\mathscr{U}_{\mathscr{K}}(H)$ acts freely (from left and right) on the fibers. Thus we obtain a fibration $\mathscr{U}_{\mathscr{K}}(H) \rightarrow \mathscr{U}_{\text {Fred }}(H) \rightarrow \mathscr{U}_{*} \mathscr{Q}(H)$. The claim now follows since $\mathscr{U}_{*} \mathscr{Q}(H)$ is contractible.

To see the latter we note that for any $C^{*}$-algebra $\mathscr{A}$ the set $\{u \in \mathscr{A} \mid u$ unitary, $-1 \notin$ spec $u\}$ is contractible. The contraction is given by $H_{t}(u):=\exp (t \log u), 0 \leq t \leq 1$. This is well-defined since $-1 \notin \operatorname{spec} u$.

2. Let $H=\operatorname{ker}(U+I) \oplus H_{1}=: H_{0} \oplus H_{1}$. Then $U$ splits into $U=-I_{H_{0}} \oplus \widetilde{U}$ and $-1 \notin \operatorname{spec} \widetilde{U}$. Now put $f(t):=-I_{H_{0}} \oplus \exp (t \log \widetilde{U})$.

In view of this lemma the winding number (6.3) extends to a group isomorphism

$$
\text { wind }: \pi_{1}\left(\mathscr{U}_{\text {Fred }}(H)\right) \rightarrow \mathbb{Z} \text {. }
$$

Next we define the winding number for not necessarily closed paths in $\mathscr{U}_{\text {Fred }}(H)$. Namely, as it was noted in the previous proof the space $\mathscr{U}_{*}(H)$ is contractible. Hence the natural map $\pi_{1}\left(\mathscr{U}_{\text {Fred }(H)}\right) \rightarrow \pi_{1}\left(\mathscr{U}_{\text {Fred }}(H), \mathscr{U}_{*}(H)\right)$ is a bijection and thus we obtain a winding number defined for curves $f:([0,1],\{0,1\}) \rightarrow\left(\mathscr{U}_{\text {Fred }}(H), \mathscr{U}_{*}(H)\right)$. 
More concretely, if $f$ is such a curve then one chooses $\tilde{f}:[0,1] \rightarrow \mathscr{U}_{*}(H)$ with $\tilde{f}(0)=f(1)$ and $\tilde{f}(1)=f(0)$. Then $f * \tilde{f}$ is a closed curve in $\mathscr{U}_{\text {Fred }}(H)$ and one puts wind $(f):=\operatorname{wind}(\widetilde{f} * f)$. Since $\mathscr{U}_{*}(H)$ is contractible it is clear that wind $(f)$ is well-defined independently of the choice of $\tilde{f}$.

Finally, we choose a convention to define the winding number for a curve whose endpoints do not lie in $\mathscr{U}_{*}(H)$ : let $f:[0,1] \rightarrow \mathscr{U}_{\text {Fred }}(H)$ be a continuous curve. -1 is an isolated point in the spectrum of $f(t)$ since $-1 \notin \operatorname{spec}_{\mathrm{ess}}(f(t))$. We may therefore choose an $\varepsilon>0$ such that for all $\varphi \in[-\varepsilon, \varepsilon], \varphi \neq 0$, we have $-1 \notin \operatorname{spec}\left(f(j) e^{i \varphi}\right), j=0,1$. Now define

$$
\operatorname{wind}(f):=\operatorname{wind}\left(f e^{-i \varepsilon}\right) .
$$

The winding number has the following properties:

1. Path Additivity: Let $f_{1}, f_{2}:[0,1] \rightarrow \mathscr{U}_{\text {Fred }}(H)$ be continuous paths with $f_{2}(0)=$ $f_{1}(1)$. Then

$$
\operatorname{wind}\left(f_{1} * f_{2}\right)=\operatorname{wind}\left(f_{1}\right)+\operatorname{wind}\left(f_{2}\right) .
$$

2. Homotopy invariance: Let $f_{1}, f_{2}$ be continuous paths in $\mathscr{U}_{\text {Fred }}$. Assume that there is a homotopy $H:[0,1] \times[0,1] \rightarrow \mathscr{U}_{\text {Fred }}$ such that $H(0, t)=f_{1}(t), H(1, t)=f_{2}(t)$ and such that $\operatorname{dim} \operatorname{ker}(H(s, 0)+I), \operatorname{dim} \operatorname{ker}(H(s, 1)+I)$ are independent of $s$. Then wind $\left(f_{1}\right)=\operatorname{wind}\left(f_{2}\right)$.

3. If $f:[0,1] \rightarrow \mathscr{U}_{\mathrm{tr}}(H)$ is a $C^{1}$-curve then

$$
\text { wind }(f)=\frac{1}{2 \pi i}\left(\int_{0}^{1} \operatorname{tr}\left(f(t)^{-1} f^{\prime}(t)\right) d t-\operatorname{tr}(\log f(1))+\operatorname{tr}(\log f(0))\right),
$$

where the logarithm is normalized as in (4.11).

We note in passing that the winding number may be interpreted as a spectral flow across -1 [4], [28]. Namely, the winding number of a path $f:[0,1] \rightarrow \mathscr{U}_{\text {Fred }}(H)$ can be calculated as follows: choose a subdivision $0=t_{0}<t_{1}<\ldots<t_{n}=1$ and $0<\varepsilon_{j}<\pi$, $j=0, \ldots, n-1$, such that $-e^{i \varphi} \notin \operatorname{spec}_{\mathrm{ess}}(f(t))$ for $t \in\left[t_{j}, t_{j+1}\right]$ and $|\varphi| \leq \varepsilon_{j}$ and moreover $-e^{ \pm i \varepsilon_{j}} \notin \operatorname{spec} f(t)$ for $t \in\left[t_{j}, t_{j+1}\right]$. Then put

$$
\begin{aligned}
\operatorname{wind}(f(t))_{t_{j} \leq t \leq t_{j+1}}:= & \#\left(\operatorname{spec}\left(f\left(t_{j+1}\right)\right) \cap\left\{-e^{i \varphi} \mid 0<\varphi<\varepsilon_{j}\right\}\right) \\
& -\#\left(\operatorname{spec}\left(f\left(t_{j}\right)\right) \cap\left\{-e^{i \varphi} \mid 0<\varphi<\varepsilon_{j}\right\},\right.
\end{aligned}
$$

where eigenvalues are counted with multiplicity. Finally,

$$
\text { wind }(f)=\sum_{j=0}^{n-1} \operatorname{wind}(f(t))_{t_{j} \leq t \leq t_{j+1}} \text {. }
$$

Definition 6.2. Let $U \in \mathscr{U}_{\mathscr{K}}(H)$ and $V \in \mathscr{U}_{\text {Fred }}(H)$. Then the double index $\tau_{w}(U, V) \in \mathbb{Z}$ is defined as follows: choose continuous paths $f:[0,1] \rightarrow \mathscr{U}_{\mathscr{K}}(H), g:$ $[0,1] \rightarrow \mathscr{U}_{\text {Fred }}(H)$ such that $f(0)=g(0)=I$ and $f(1)=U, g(1)=V$. Then put $\tau_{w}(U, V):=\operatorname{wind}(f)+\operatorname{wind}(g)-\operatorname{wind}(f g) . \quad \tau_{w}(U, V)$ is defined accordingly if $U \in$ $\mathscr{U}_{\text {Fred }}(H), V \in \mathscr{U}_{\mathscr{K}}(H)$ or $U, V \in \mathscr{U}_{\text {Fred }}(H), U V \in \mathscr{U}_{\mathscr{K}}(H)$. 
Proposition 6.3. The double index $\tau_{w}$ is well-defined. It has the following properties:

1. (Homotopy invariance) If $f:[0,1] \rightarrow \mathscr{U}_{\mathscr{K}}(H), g:[0,1] \rightarrow \mathscr{U}_{\text {Fred }}(H)$ are continuous paths then

$$
\tau_{w}(f(1), g(1))-\tau_{w}(f(0), g(0))=\operatorname{wind}(f)+\operatorname{wind}(g)-\operatorname{wind}(f g) .
$$

In particular, if $\operatorname{dim} \operatorname{ker}(f(t)+I)$, dim $\operatorname{ker}(g(t)+I)$ and $\operatorname{dim} \operatorname{ker}(f(t) g(t)+I)$ are independent of $t$ then $\tau_{w}(f(1), g(1))=\tau_{w}(f(0), g(0))$.

2. If $U, V \in \mathscr{U}_{\mathrm{tr}}(H)$ then

$$
\tau_{w}(U, V)=\frac{1}{2 \pi i}(\operatorname{tr} \log U V-\operatorname{tr} \log U-\operatorname{tr} \log V) .
$$

3. For any $U \in \mathscr{U}_{\text {Fred }}(H)$ we have

$$
\tau_{w}(I, U)=\tau_{w}(U, I)=0 \text { and } \tau_{w}\left(U, U^{-1}\right)=-\operatorname{dim} \operatorname{ker}(U+I) .
$$

Proof. First note that if $U \in \mathscr{U}_{\mathscr{K}}(H)$ and $V \in \mathscr{U}_{\text {Fred }}(H)$ then since $U-I$ is compact one has $\operatorname{spec}_{\text {ess }}(U V)=\operatorname{spec}_{\text {ess }}(V)$, in particular $U V \in \mathscr{U}_{\text {Fred }}(H)$. If $\tilde{f}:[0,1] \rightarrow$ $\mathscr{U}_{\mathscr{K}}(H), \widetilde{g}:[0,1] \rightarrow \mathscr{U}_{\text {Fred }}(H)$ are different paths with $\tilde{f}(0)=\widetilde{g}(0)=I, \widetilde{f}(1)=U, \widetilde{g}(1)=$ $V$ then consider the closed paths $f * \widetilde{f}_{-}$and $g * \widetilde{g}_{-}$, where $\widetilde{f}_{-}$denotes the path $\widetilde{f}$ traversed in the opposite direction. Since the pointwise product of closed paths $\left(f * \widetilde{f}_{-}\right)\left(g * \widetilde{g}_{-}\right)$ is homotopic to $\left(f * \widetilde{f}_{-}\right) *\left(g * \widetilde{g}_{-}\right)$we find

$$
\begin{aligned}
0 & =\operatorname{wind}\left(f * \widetilde{f}_{-}\right)+\operatorname{wind}\left(g * \widetilde{g}_{-}\right)-\operatorname{wind}\left(\left(f * \widetilde{f}_{-}\right)\left(g * \widetilde{g}_{-}\right)\right) \\
& =-\operatorname{wind}(\widetilde{f})+\operatorname{wind}(f)-\operatorname{wind}(\widetilde{g})+\operatorname{wind}(g)+\operatorname{wind}(\widetilde{f} \widetilde{g})-\operatorname{wind}(f g) .
\end{aligned}
$$

This shows that $\tau_{w}$ is well-defined. The homotopy invariance is straightforward from the definition and the homotopy invariance of the winding number.

2. This assertion is a consequence of (6.5).

3. That $\tau_{w}(I, U)=\tau_{w}(U, I)=0$ follows immediately from the definition.

For $U \in \mathscr{U}_{\mathrm{tr}}(H)$ the third identity follows from Assertion 2. (note the normalization (4.11) of $\log$ ). If $U$ is arbitrary we apply Lemma 6.1 2. and choose a continuous path $f:[0,1] \rightarrow \mathscr{U}_{\text {Fred }}(H)$ such that $f(1)=U, f(0) \in \mathscr{U}_{\text {tr }}(H)$ and such that $\operatorname{dim} \operatorname{ker}(f(t)+I)$ is independent of $t$. The claim now follows from the homotopy invariance 1.

A priori $\tau_{w}$ cannot be defined on $\mathscr{U}_{\text {Fred }}(H) \times \mathscr{U}_{\text {Fred }}(H)$ (which might be desirable) since for $U, V \in \mathscr{U}_{\text {Fred }}(H)$ in general $U V \notin \mathscr{U}_{\text {Fred }}(H)$. Even if one assumes $U V \in$ $\mathscr{U}_{\text {Fred }}(H)$ it is in general not possible to choose paths $f, g$ as above such that $f(t) g(t) \in$ $\mathscr{U}_{\text {Fred }}(H)$ for all $t$.

Corollary 6.4. Let $f:[0,1] \rightarrow \mathscr{U}_{\text {Fred }}(H)$ be a continuous path. Then

$$
\text { wind }(f)+\operatorname{wind}\left(f^{-1}\right)=\operatorname{dim} \operatorname{ker}(f(0)+I)-\operatorname{dim} \operatorname{ker}(f(1)+I) .
$$

Proof. We apply Proposition 6.3 1. with $g=f^{-1}$ and obtain using Proposition 6.33.

$$
\begin{aligned}
\operatorname{wind}(f)+\operatorname{wind}\left(f^{-1}\right) & =\tau_{w}\left(f(1), f(1)^{-1}\right)-\tau_{w}\left(f(0), f(0)^{-1}\right) \\
& =-\operatorname{dim} \operatorname{ker}(f(1)+I)+\operatorname{dim} \operatorname{ker}(f(0)+I) .
\end{aligned}
$$


6.2. Maslov Index. Let $(H,\langle\rangle,, \gamma)$ be a Hermitian symplectic Hilbert space (cf. Def. 2.8). Thus $\gamma: H \rightarrow H$ is a unitary map satisfying $\gamma^{2}=-1$ and the eigenspaces $\mathscr{E}_{ \pm i}:=\operatorname{ker}(\gamma \mp i)$ have the same Hilbert space dimension. As in Section 2 we denote by

$$
\mathscr{L}:=\left\{L \subset H \mid L \text { closed subspace, } \gamma L=L^{\perp}\right\}
$$

the set of Lagrangian subspaces. As usual $L \in \mathscr{L}$ will be identified with the orthogonal projection $P_{L}$ onto $L$. The image of an orthogonal projection $P$ in $H$ is Lagrangian if and only if $\gamma P \gamma^{*}=I-P$. Similarly as in Section 2 we put

$$
\begin{aligned}
\operatorname{Gr}(H) & :=\left\{P \in \mathscr{B}(H) \mid P=P^{*}, P^{2}=P, \gamma P \gamma^{*}=I-P\right\}, \\
\operatorname{Gr}_{\text {Fred }}^{(2)}(H) & :=\{(P, Q) \mid P, Q \in \operatorname{Gr}(H),(P, Q) \text { are a Fredholm pair }\}, \\
\operatorname{Gr}_{*}^{(2)}(H) & :=\{(P, Q) \mid P, Q \in \operatorname{Gr}(H),(P, Q) \text { is an invertible pair }\}, \\
\operatorname{Gr}_{\mathscr{K}}^{(2)}(H) & :=\{(P, Q) \mid P, Q \in \operatorname{Gr}(H), P-Q \text { is compact }\} .
\end{aligned}
$$

Notice that, in contrast to the definition of $\operatorname{Gr}(A)$, there is no Fredholm assumption about elements of $\operatorname{Gr}(H)$. The corresponding spaces of Lagrangians are

$$
\begin{aligned}
\mathscr{L}_{\text {Fred }}^{(2)} & :=\left\{\left(L_{1}, L_{2}\right) \mid L_{1}, L_{2} \in \mathscr{L},\left(L_{1}, L_{2}\right) \text { is Fredholm }\right\}, \\
\mathscr{L}_{*}^{(2)} & :=\left\{\left(L_{1}, L_{2}\right) \in \mathscr{L}^{(2)} \mid\left(L_{1}, L_{2}\right) \text { is invertible }\right\} .
\end{aligned}
$$

Recall that a pair of Lagrangian spaces $\left(L_{1}, L_{2}\right)$ is Fredholm if $L_{1} \cap L_{2}$ is finitedimensional and if $L_{1}+L_{2}$ is closed with finite codimension, and that the pair $\left(L_{1}, L_{2}\right)$ is invertible if $L_{1} \cap L_{2}=\{0\}$ and $L_{1}+L_{2}=H$.

We emphasize the confusing fact that $\left(L_{1}, L_{2}\right)$ is Fredholm (resp. invertible) if and only if the pair of projections $\left(I-P_{L_{1}}, P_{L_{2}}\right)$ is Fredholm (resp. invertible). Therefore, a Fredholm pair of projections $(P, Q)$ will sometimes be identified with the pair $($ ker $P, \operatorname{im} Q)$ of Lagrangian subspaces.

As in Lemma 2.6 one sees that with respect to the decomposition $H=\mathscr{E}_{i} \oplus \mathscr{E}_{-i}$ each $P \in \operatorname{Gr}(H)$ takes the form

$$
P=\frac{1}{2}\left(\begin{array}{cc}
I & \Phi(P)^{*} \\
\Phi(P) & I
\end{array}\right)
$$

where $\Phi(P) \in \mathscr{U}\left(\mathscr{E}_{i}, \mathscr{E}_{-i}\right)$. Moreover, the map

$$
\Phi: \operatorname{Gr}(H) \longrightarrow \mathscr{U}\left(\mathscr{E}_{i}, \mathscr{E}_{-i}\right)
$$

is a diffeomorphism. Furthermore, the pair $(P, Q)$ is Fredholm if and only if $\Phi(P) \Phi(Q)^{*} \in \mathscr{U}_{\text {Fred }}\left(\mathscr{E}_{-i}\right)$ and $(P, Q)$ is invertible if and only if $\Phi(P) \Phi(Q)^{*} \in \mathscr{U}_{*}\left(\mathscr{E}_{-i}\right)$ (cf. Lemma 2.6). Finally, $P-Q$ is compact (resp. trace class) if and only if $\Phi(P) \Phi(Q)^{*} \in \mathscr{U}_{\mathscr{K}}\left(\mathscr{E}_{-i}\right)\left(\operatorname{resp} . \mathscr{U}_{\mathrm{tr}}\left(\mathscr{E}_{-i}\right)\right)$. 
Proposition 6.5. There are diffeomorphisms

$$
\begin{aligned}
\operatorname{Gr}_{\text {Fred }}^{(2)}(H) & \cong \mathscr{U}_{\text {Fred }}\left(\mathscr{E}_{-i}\right) \times \mathscr{U}\left(\mathscr{E}_{i}, \mathscr{E}_{-i}\right), \\
\operatorname{Gr}_{\mathscr{K}}^{(2)}(H) & \cong \mathscr{U}_{\mathscr{K}}\left(\mathscr{E}_{-i}\right) \times \mathscr{U}\left(\mathscr{E}_{i}, \mathscr{E}_{-i}\right), \\
\operatorname{Gr}_{*}^{(2)}(H) & \cong \mathscr{U}_{*}\left(\mathscr{E}_{-i}\right) \times \mathscr{U}\left(\mathscr{E}_{i}, \mathscr{E}_{-i}\right), \\
\operatorname{Gr}_{\mathscr{K}}^{(2)}(H) \cap \operatorname{Gr}_{*}^{(2)}(H) & \cong\left(\mathscr{U}_{\mathscr{K}}\left(\mathscr{E}_{-i}\right) \cap \mathscr{U}_{*}\left(\mathscr{E}_{-i}\right)\right) \times \mathscr{U}\left(\mathscr{E}_{i}, \mathscr{E}_{-i}\right) .
\end{aligned}
$$

In particular the identifications induce homotopy equivalences

$$
\begin{aligned}
\left(\operatorname{Gr}_{\mathrm{Fred}}^{(2)}(H), \mathrm{Gr}_{*}^{(2)}(H)\right) & \simeq\left(\mathscr{U}_{\mathrm{Fred}}\left(\mathscr{E}_{-i}\right), \mathscr{U}_{*}\left(\mathscr{E}_{-i}\right)\right) \\
\left(\operatorname{Gr}_{\mathscr{K}}^{(2)}(H), \operatorname{Gr}_{*}^{(2)}(H) \cap \operatorname{Gr}_{\mathscr{K}}(H)\right) & \simeq\left(\mathscr{U}_{\mathscr{K}}\left(\mathscr{E}_{-i}\right), \mathscr{U}_{*}\left(\mathscr{E}_{-i}\right) \cap \mathscr{U}_{\mathscr{K}}\left(\mathscr{E}_{-i}\right)\right) .
\end{aligned}
$$

ProOF. In all four cases the diffeomorphism is given by

$$
(P, Q) \mapsto\left(\Phi(P) \Phi(Q)^{*}, \Phi(P)\right) .
$$

By Kuiper's Theorem [22] the space $\mathscr{U}\left(\mathscr{E}_{i}, \mathscr{E}_{-i}\right)$ is contractible and hence we obtain the claimed homotopy equivalences.

The Maslov Index [10], [26] is an integer invariant of Fredholm pairs of paths of Lagrangian subspaces. We discuss it in terms of the projection picture of Lagrangian subspaces. Let $(f, g):[0,1] \rightarrow \operatorname{Gr}_{\text {Fred }}^{(2)}(H)$ be a continuous path (i.e. a pair of paths in $\operatorname{Gr}(H)$ such that $(f(t), g(t))$ is Fredholm for all $t)$. The Maslov index Mas $(f, g)$ is the algebraic count of how many times ker $f(t)$ passes through im $g(t)$ along the path. We use the notation $\operatorname{Mas}(f, g), \operatorname{Mas}(\operatorname{ker} f, \operatorname{im} g), \operatorname{Mas}(\operatorname{im} f, \operatorname{ker} g)$ interchangeably. Indeed $\operatorname{Mas}(\operatorname{ker} f, \operatorname{im} g)=\operatorname{Mas}(\gamma \operatorname{ker} f, \gamma \operatorname{im} g)=\operatorname{Mas}(\operatorname{im} f, \operatorname{ker} g)$.

The Maslov index has the following properties (cf. [26], [10]):

1. Path Additivity: Let $\left(f_{j}, g_{j}\right):[0,1] \rightarrow \operatorname{Gr}_{\text {Fred }}^{(2)}(H), j=1,2$, be continuous paths with $f_{2}(0)=f_{1}(1), g_{2}(0)=g_{1}(1)$ then

$$
\operatorname{Mas}\left(\left(f_{1}, g_{1}\right) *\left(f_{2}, g_{2}\right)\right)=\operatorname{Mas}\left(f_{1}, g_{1}\right)+\operatorname{Mas}\left(f_{2}, g_{2}\right) \text {. }
$$

2. Homotopy Invariance: Let $\left(f_{j}, g_{j}\right):[0,1] \rightarrow \operatorname{Gr}_{\text {Fred }}^{(2)}(H), j=0,1$, such that $\left(f_{0}, g_{0}\right)$ is homotopic $\left(f_{1}, g_{1}\right)$ rel endpoints then

$$
\operatorname{Mas}\left(f_{0}, g_{0}\right)=\operatorname{Mas}\left(f_{1}, g_{1}\right) \text {. }
$$

More generally, suppose that $(F, G)$ is a homotopy from $\left(f_{0}, g_{0}\right)=$ $(F(-, 0), G(-, 0))$ to $\left(f_{1}, g_{1}\right)=(F(-, 1), G(-, 1))$ and suppose that $\operatorname{dim}(\operatorname{ker} F(0, s) \cap \operatorname{im} G(0, s))$ and $\operatorname{dim}(\operatorname{ker} F(1, s) \cap \operatorname{im} G(1, s))$ are independent of $s \in[0,1]$. Then $\operatorname{Mas}\left(f_{0}, g_{0}\right)=\operatorname{Mas}\left(f_{1}, g_{1}\right)$.

3. Normalization: This is done in two steps. First one requires that on paths with endpoints in $\operatorname{Gr}_{*}^{(2)}(H)$ the Maslov index induces a group isomorphism $\pi_{1}\left(\operatorname{Gr}_{\text {Fred }}^{(2)}(H), \operatorname{Gr}_{*}^{(2)}(H)\right) \rightarrow \mathbb{Z}\left(\right.$ since $\operatorname{Gr}_{*}^{(2)}(H) \simeq \mathscr{U}_{*}\left(\mathscr{E}_{-i}\right) \times \mathscr{U}\left(\mathscr{E}_{i}, \mathscr{E}_{-i}\right)$ is contractible $\pi_{1}\left(\operatorname{Gr}_{\text {Fred }}^{(2)}(H), \operatorname{Gr}_{*}^{(2)}(H)\right)$ is indeed a group). This determines Mas on paths with endpoints in $\operatorname{Gr}_{*}^{(2)}(H)$ up to a sign. The sign is chosen as follows: if $(P, Q) \in \operatorname{Gr}^{(2)}(H)$ then $\operatorname{Mas}\left(e^{t \gamma} P e^{-t \gamma}, Q\right)_{-\varepsilon \leq t \leq \varepsilon}=\operatorname{dim}(\operatorname{ker} P \cap \operatorname{im} Q)$ for $\varepsilon$ small enough. 
Secondly, if $(f, g):[0,1] \rightarrow \operatorname{Gr}_{\text {Fred }}^{(2)}(H)$ is an arbitrary continuous path then one chooses $\varepsilon$ small enough such that the pairs $\left(e^{s \gamma} f(j) e^{-s \gamma}, g(j)\right)$ are invertible for $j=0,1,0<s \leq \varepsilon$. Then one puts

$$
\operatorname{Mas}(f, g):=\operatorname{Mas}\left(e^{\varepsilon \gamma} f e^{-\varepsilon \gamma}, g\right) .
$$

Actually, the normalization property 3. determines the Maslov index completely and it may be viewed as its definition. Properties 1. and 2. follow from 3. There exist other conventions for dealing with paths whose endpoints do not lie in $\operatorname{Gr}_{*}^{(2)}(H)$ and not all of these conventions satisfy path additivity.

The discussion of the Maslov index works as well when the Hermitian symplectic Hilbert space $H$ is finite-dimensional. In this case the Fredholm condition is vacuous and the Maslov index is defined on $\operatorname{Gr}^{(2)}(H)=\operatorname{Gr}(H) \times \operatorname{Gr}(H)$. We will use the Maslov index in both contexts; in the infinite-dimensional setting with $H=L^{2}\left(E_{\mid \partial X}\right)$ and in the finite-dimensional context with $H=\operatorname{ker} A$.

TheOREM 6.6. For a continuous path $(f, g)$ in $\operatorname{Gr}_{\mathrm{Fred}}^{(2)}(H)$ the Maslov index is related to the winding number by the equation

$$
\operatorname{Mas}(f, g)=-\operatorname{wind}\left(\Phi(f) \Phi(g)^{*}\right) .
$$

Proof. In view of Proposition 6.5 the right hand side of (6.15) induces a group isomorphism $\pi_{1}\left(\mathrm{Gr}_{\text {Fred }}^{(2)}(H), \mathrm{Gr}_{*}^{(2)}(H)\right) \rightarrow \mathbb{Z}$. It remains therefore to check the sign convention and the convention for paths with endpoints not in $\operatorname{Gr}_{*}^{(2)}(H)$. Let $(P, Q) \in$ $\operatorname{Gr}_{\text {Fred }}^{(2)}(H)$. Then, by definition, $\operatorname{Mas}\left(e^{t \gamma} P e^{-t \gamma}, Q\right)_{-\varepsilon \leq t \leq \varepsilon}=\operatorname{dim}(\operatorname{ker} P \cap i m Q)$ for $\varepsilon$ small enough. A straightforward calculation shows

$$
\Phi\left(e^{s \gamma} P e^{-s \gamma}\right)=e^{-2 s \gamma} \Phi(P)
$$

and thus for $\varepsilon>0$ small enough we have, in view of Lemma 2.6 (3),

$$
\begin{aligned}
\operatorname{wind}\left(\Phi\left(e^{s \gamma} P e^{-s \gamma}\right) \Phi(Q)^{*}\right)_{-\varepsilon \leq s \leq \varepsilon} & =\operatorname{dim}(\operatorname{ker} P \cap \operatorname{im} Q) \text { wind }\left(-e^{-2 i s}\right)_{-\varepsilon \leq s \leq \varepsilon} \\
& =-\operatorname{dim}(\operatorname{ker} P \cap \operatorname{im} Q) .
\end{aligned}
$$

To check the convention for paths with endpoints not in $\operatorname{Gr}_{*}^{(2)}(H)$ we consider the paths $\left(e^{s \gamma} P e^{-s \gamma}, Q\right),-\varepsilon \leq s \leq 0$, resp. $0 \leq s \leq \varepsilon$. By definition we have for $\delta>0$ small enough

$$
\begin{aligned}
\operatorname{Mas}\left(e^{s \gamma} P e^{-s \gamma}, Q\right)_{-\varepsilon \leq s \leq 0} & =\operatorname{Mas}\left(e^{(\delta+s) \gamma} P e^{-(\delta+s) \gamma}, Q\right)_{-\varepsilon \leq s \leq 0} \\
& =\operatorname{Mas}\left(e^{t \gamma} P e^{-t \gamma}, Q\right)_{-\varepsilon+\delta \leq s \leq+\delta}=\operatorname{dim}(\operatorname{ker} P \cap \operatorname{im} Q),
\end{aligned}
$$

and, analogously,

$$
\operatorname{Mas}\left(e^{s \gamma} P e^{-s \gamma}, Q\right)_{0 \leq s \leq \varepsilon}=0
$$


According to our convention for the winding number we have, on the other hand,

$$
\begin{gathered}
\operatorname{wind}\left(-e^{-2 i s}\right)_{-\varepsilon \leq s \leq 0}=-1, \\
\operatorname{wind}\left(-e^{2 i s}\right)_{0 \leq s \leq \varepsilon}=0 .
\end{gathered}
$$

In view of (6.17) the proof is complete.

Corollary 6.7. Let $(f, g)$ be a continuous path in $\operatorname{Gr}_{\text {Fred }}^{(2)}(H)$.

(1) The Maslov index Mas $_{-\gamma}$ with respect to the opposite symplectic structure is related to $\operatorname{Mas}_{\gamma}$ as follows: $\operatorname{Mas}_{-\gamma}(f, g)=\operatorname{Mas}_{\gamma}(g, f)$.

(2) $\operatorname{Mas}_{\gamma}(f, g)+\operatorname{Mas}_{\gamma}(g, f)=\operatorname{dim}(\operatorname{ker} f(1) \cap \operatorname{im} g(1))-\operatorname{dim}(\operatorname{ker} f(0) \cap \operatorname{im} g(0))$.

Proof. (1) In view of (5.6) and the previous theorem we find $\operatorname{Mas}_{-\gamma}(f, g)=$ $-\operatorname{wind}\left(\Phi_{-\gamma}(f) \Phi_{-\gamma}(g)^{*}\right)=-\operatorname{wind}\left(\Phi_{\gamma}(f)^{*} \Phi_{\gamma}(g)\right)=\operatorname{wind}\left(\Phi_{\gamma}(g) \Phi_{\gamma}(f)^{*}\right)=\operatorname{Mas}_{\gamma}(g, f)$.

(2) Using the previous Theorem and Corollary 6.4 we obtain (we write Mas instead of $\operatorname{Mas}_{\gamma}$ )

$$
\begin{aligned}
\operatorname{Mas}(f, g)+\operatorname{Mas}(g, f) & =-\operatorname{wind}\left(\Phi(f) \Phi(g)^{*}\right)-\operatorname{wind}\left(\left(\Phi(f) \Phi(g)^{*}\right)^{-1}\right) \\
& =\operatorname{dim}(\operatorname{ker} f(1) \cap \operatorname{im} g(1))-\operatorname{dim}(\operatorname{ker} f(0) \cap \operatorname{im} g(0)) .
\end{aligned}
$$

Finally we construct a version of the Maslov triple index in our context. The Maslov triple index as defined in (cf. [10, Sec. 8]) cannot be generalized to the present infinite-dimensional setting. The reason is simply that interesting triples of Lagrangian subspaces $L_{1}, L_{2}, L_{3}$ such that $\left(L_{1}, L_{2}\right),\left(L_{2}, L_{3}\right),\left(L_{3}, L_{1}\right)$ are all Fredholm pairs are hard to find.

However, motivated by [10, Prop. 8.4] we can construct a variant $\tau_{\mu}$ of the Maslov triple index which is related to the double index $\tau_{w}$ : consider continuous paths $f, g, h$ : $[0,1] \rightarrow \operatorname{Gr}(H)$ such that $(f, g),(g, h),(f, h)$ map into $\operatorname{Gr}_{\text {Fred }}^{(2)}(H)$ and such that $f-g$ or $g-h$ or $f-h$ maps into the set of compact operators. If, say, $f(t)-g(t)$ is compact for all $t$ then, of course, it suffices to assume that $(f(t), h(t))$ is Fredholm for all $t$. The Fredholmness of $(f(t), g(t)),(g(t), h(t))$ then follows. Now in view of Theorem 6.6 and Proposition 6.3 we find

$$
\begin{aligned}
& \operatorname{Mas}(f, g)+\operatorname{Mas}(g, h)-\operatorname{Mas}(f, h) \\
& =-\operatorname{wind}\left(\Phi(f) \Phi(g)^{*}\right)-\operatorname{wind}\left(\Phi(g) \Phi(h)^{*}\right)+\operatorname{wind}\left(\Phi(f) \Phi(h)^{*}\right) \\
& =-\tau_{w}\left(\Phi(f(1)) \Phi(g(1))^{*}, \Phi(g(1)) \Phi(h(1))^{*}\right) \\
& \quad+\tau_{w}\left(\Phi(f(0)) \Phi(g(0))^{*}, \Phi(g(0)) \Phi(h(1))^{*}\right) .
\end{aligned}
$$

This motivates the following definition.

Definition 6.8. Let $P, Q, R \in \operatorname{Gr}(H)$ such that $(P, Q),(Q, R),(P, R)$ are Fredholm and at least one of the differences $P-Q, Q-R, P-R$ is compact. Then we set

$$
\tau_{\mu}(P, Q, R):=-\tau_{w}\left(\Phi(P) \Phi(Q)^{*}, \Phi(Q) \Phi(R)^{*}\right) .
$$

and call $\tau_{\mu}$ the triple index of $(P, Q, R)$. 
The triple index $\tau_{\mu}$ inherits properties from $\tau_{w}$ in a straightforward way. For example, one has the following.

Lemma 6.9. Let $P, Q, R \in \operatorname{Gr}(H)$ such that $P-Q, Q-R$ are trace class. Then

$$
\begin{gathered}
\tau_{\mu}(P, Q, R)=\frac{1}{2 \pi i}\left(\operatorname{tr} \log \left(\Phi(P) \Phi(Q)^{*}\right)+\operatorname{tr} \log \left(\Phi(Q) \Phi(R)^{*}\right)\right. \\
\left.-\operatorname{tr} \log \left(\Phi(P) \Phi(R)^{*}\right)\right) .
\end{gathered}
$$

We will have occasion below to use the homotopy invariance of the triple index.

Lemma 6.10. Let $P, Q, R:[0,1] \rightarrow \operatorname{Gr}(H)$ be paths in $\operatorname{Gr}(H)$ so that $(P, Q),(Q, R),(P, R)$ map into $\operatorname{Gr}_{\text {Fred }}^{(2)}(H)$ and at least one of the differences $P-Q, Q-R, P-R$ maps into the set of compact operators. Suppose further that $\operatorname{dim}(\operatorname{ker} P(t) \cap \operatorname{im} Q(t)), \operatorname{dim}(\operatorname{ker} Q(t) \cap \operatorname{im} R(t))$, and $\operatorname{dim}(\operatorname{ker} P(t) \cap \operatorname{im} R(t))$ are independent of $t$. Then

$$
\tau_{\mu}(P(0), Q(0), R(0))=\tau_{\mu}(P(1), Q(1), R(1)) .
$$

Proof. By (6.21) we have

$$
\begin{gathered}
\tau_{\mu}(P(0), Q(0), R(0))-\tau_{\mu}(P(1), Q(1), R(1)) \\
\quad=\operatorname{Mas}(P, Q)+\operatorname{Mas}(Q, R)-\operatorname{Mas}(P, R) .
\end{gathered}
$$

Now the claim follows immediately from the homotopy invariance of the Maslov index.

We defined the triple index in such a way that formulas become short. A drawback of this is that $\tau_{\mu}$ is not antisymmetric in the variables, as the following proposition shows.

Proposition 6.11. Let $P, Q, R \in \operatorname{Gr}(H)$ such that $(P, Q),(Q, R),(P, R)$ are Fredholm and at least one of the differences is compact. Then

$$
\begin{aligned}
\tau_{\mu}(P, R, Q) & =-\tau_{\mu}(P, Q, R)+\operatorname{dim}(\operatorname{ker} Q \cap \operatorname{im} R), \\
\tau_{\mu}(Q, P, R) & =-\tau_{\mu}(P, Q, R)+\operatorname{dim}(\operatorname{ker} P \cap \operatorname{im} Q), \\
\tau_{\mu}(R, Q, P) & =-\tau_{\mu}(P, Q, R)+\operatorname{dim}(\operatorname{ker} P \cap \operatorname{im} Q) \\
& \quad+\operatorname{dim}(\operatorname{ker} Q \cap \operatorname{im} R)-\operatorname{dim}(\operatorname{ker} P \cap \operatorname{im} R) .
\end{aligned}
$$

Moreover,

$$
\tau_{\mu}(P, P, Q)=\tau_{\mu}(Q, P, P)=0 \text { and } \tau_{\mu}(P, Q, P)=\operatorname{dim}(\operatorname{ker} P \cap \operatorname{im} Q) .
$$

Proof. We prove (6.26) first. From Proposition 6.3 and the definition of $\tau_{\mu}$ we infer

$$
\begin{aligned}
\tau_{\mu}(P, P, Q) & =-\tau_{w}\left(I, \Phi(P) \Phi(Q)^{*}\right)=0, \\
\tau_{\mu}(Q, P, P) & =-\tau_{w}\left(\Phi(Q) \Phi(P)^{*}, I\right)=0, \\
\tau_{\mu}(P, Q, P) & =-\tau_{w}\left(\Phi(P) \Phi(Q)^{*},\left(\Phi(P) \Phi(Q)^{*}\right)^{-1}\right)=\operatorname{dim}(\operatorname{ker} P \cap \operatorname{im} Q) .
\end{aligned}
$$


To prove (6.25) we assume, without loss of generality, that $Q-R$ is compact. Let $f(t):=(1-t) Q+t R, 0 \leq t \leq 1$. Then we obtain from Corollary 6.7 and (6.26)

$$
\begin{aligned}
\tau_{\mu}(P, R, Q)= & \tau_{\mu}(P, R, Q)-\tau_{\mu}(P, Q, Q)=\operatorname{Mas}(P, f)+\operatorname{Mas}(f, Q) \\
= & \operatorname{Mas}(P, f)-\operatorname{Mas}(Q, f)+\operatorname{dim}(\operatorname{ker} Q \cap \operatorname{im} R) \\
= & -\tau_{\mu}(P, Q, R)+\operatorname{dim}(\operatorname{ker} Q \cap \operatorname{im} R), \\
\tau_{\mu}(Q, P, R)= & \operatorname{Mas}(P, f)-\operatorname{Mas}(Q, f)+\tau_{\mu}(Q, P, Q) \\
= & -\tau_{\mu}(P, Q, R)+\operatorname{dim}(\operatorname{ker} P \cap \operatorname{im} Q), \\
\tau_{\mu}(R, Q, P)= & \operatorname{Mas}(f, Q)-\operatorname{Mas}(f, P) \\
= & -\operatorname{Mas}(Q, f)+\operatorname{Mas}(P, f)+\operatorname{dim}(\operatorname{ker} P \cap \operatorname{im} Q) \\
& \quad+\operatorname{dim}(\operatorname{ker} Q \cap \operatorname{im} R)-\operatorname{dim}(\operatorname{ker} P \cap \operatorname{im} R) .
\end{aligned}
$$

6.3. Symplectic reduction. We conclude this section with a discussion of symplectic reduction in our infinite-dimensional context. We will use symplectic reduction in Section 8 .

Let $(H,\langle.,\rangle,. \gamma)$ be a Hermitian symplectic Hilbert space with symplectic form $\omega(x, y)=\langle x, \gamma y\rangle$. For a subspace $U \subset H$ the annihilator of $U$ is defined to be

$$
\operatorname{Ann}(U):=\{x \in H \mid \forall y \in U \omega(x, y)=0\}=(\gamma U)^{\perp} .
$$

A subspace $U \subset H$ is called isotropic if $U \subset \operatorname{Ann}(U)$.

Assume for the moment that $H$ is finite-dimensional and that $\operatorname{Ann}(U) \subset U$. Then $\omega$ induces a symplectic structure on the quotient $U / \operatorname{Ann}(U)$ in a natural way. Moreover, if $L \subset H$ is Lagrangian then $R_{U}(L):=L \cap U / L \cap \operatorname{Ann}(U)$ is Lagrangian in $U / \operatorname{Ann}(U)$. $R_{U}(L)$ is called the symplectic reduction of $L$.

Proposition 6.12. Let $(H,\langle.,\rangle,. \gamma)$ be a Hermitian symplectic Hilbert space, $U \subset$ $H$ a closed subspace with $\operatorname{Ann}(U) \subset U$.

Suppose that $L \subset H$ is a Lagrangian subspace such that $L+\operatorname{Ann}(U)$ is a closed subspace of $H$. Then $(U \cap \gamma U,\langle.,\rangle,. \gamma)$ is a Hermitian symplectic Hilbert space and the orthogonal projection

$$
P_{L, U}:=\operatorname{proj}_{U \cap \gamma U}: L \cap U \longrightarrow U \cap \gamma U
$$

has closed range isomorphic to $L \cap U / L \cap \operatorname{Ann}(U)$. Moreover, $R_{U}(L)=\operatorname{im} P_{L, U}$ is Lagrangian in $U \cap \gamma U$.

$R_{U}(L)$ is called the symplectic reduction of $L$ with respect to $U$.

REMARK 6.13.

1. For $(U \cap \gamma U,\langle.,\rangle,. \gamma)$ to be Hermitian symplectic it is crucial that there is at least one Lagrangian subspace $L \subset H$ with $L+\operatorname{Ann}(U)$ closed. To illustrate the problem start with an infinite-dimensional Hermitian symplectic Hilbert space $(H,\langle.,\rangle,. \gamma)$. Let $\widetilde{H}:=H \oplus H_{1}$, where $H_{1}$ is another Hilbert space, and put $\widetilde{\gamma}:=$ $\gamma \oplus i$. Furthermore, pick a symplectic subspace $L \subset H$ and put $U:=L \oplus H_{1} \subset \widetilde{H}$. 
Then $\operatorname{Ann}(U)=L \oplus 0$ and $U \cap \gamma U=0 \oplus H_{1}$. Since $\widetilde{\gamma}$ acts by multiplication by $i$ on $U \cap \gamma U$ we conclude that $(U \cap \gamma U,\langle.,\rangle,. \widetilde{\gamma})$ is not Hermitian symplectic. From the proposition we infer that for each Lagrangian subspace $K \subset \widetilde{H}$ the space $K+\operatorname{Ann}(U)$ is not closed.

2. Proposition 6.12 in particular applies if $(L, \operatorname{Ann}(U))$ is a Fredholm pair of subspaces.

3. The assignment $L \mapsto R_{U}(L)$ is not continuous, but is continuous along paths $L_{t}$ so that $\operatorname{dim}\left(L_{t} \cap \operatorname{Ann}(U)\right)$ is constant. These facts are well-known and we omit the examples.

Proof. Certainly $U \cap \gamma U$ is a Hilbert space and $\gamma$ leaves $U \cap \gamma U$ invariant. If we can prove that im $P_{L, U}$ is Lagrangian in $U \cap \gamma U$ then from Lemma 2.7 we infer $\operatorname{dim}(\operatorname{ker}(\gamma+i) \cap U \cap \gamma U)=\operatorname{dim}(\operatorname{ker}(\gamma-i) \cap U \cap \gamma U)$.

What remains, therefore, is to prove the second part of Proposition 6.12 without using the fact that $\operatorname{dim}(\operatorname{ker}(\gamma+i) \cap U \cap \gamma U)=\operatorname{dim}(\operatorname{ker}(\gamma-i) \cap U \cap \gamma U)$.

We note first that we have an orthogonal direct sum decomposition

$$
\operatorname{Ann}(U) \oplus(U \cap \gamma U)=U .
$$

Also, im $P_{L, U}$ is an isotropic subspace of $U \cap \gamma U$. In fact, if $x \in L \cap U$ then, since $L$ is Lagrangian, $\langle x, \gamma x\rangle=0$. Writing $x=\xi+\eta, \xi \in U \cap \gamma U, \eta \in \operatorname{Ann}(U)$ then $0=\langle x, \gamma x\rangle=\langle\xi, \gamma x\rangle=\langle\xi, \xi\rangle=\left\langle P_{L, U} x, \gamma P_{L, U} x\right\rangle$.

Next consider $\xi \in U \cap \gamma U$ such that $\gamma(\xi) \perp \operatorname{im} P_{L, U}$. Thus for all $x \in L \cap U$ we have $\langle\gamma(\xi), x\rangle=\left\langle\gamma(\xi), P_{L, U} x\right\rangle=0$. Hence $\gamma(\xi) \in(L \cap U)^{\perp}=\overline{L^{\perp}+U^{\perp}}=\overline{\gamma(L)+U^{\perp}}$ and consequently, since $L+\operatorname{Ann}(U)$ is closed, $\xi \in L+\operatorname{Ann}(U)$. We may write $\xi=$ $l+\eta, l \in L, \eta \in \operatorname{Ann}(U)$. From $\xi \in U, \eta \in \operatorname{Ann}(U) \subset U$ we infer $l \in L \cap U$ and hence $\xi=P_{L, U}(l) \in \operatorname{im} P(L)$.

Summing up we have proved $\gamma\left(\left(\operatorname{im} P_{L, U}\right)^{\perp}\right) \subset \operatorname{im} P_{L, U}$. Since im $P_{L, U}$ is isotropic this implies $\operatorname{im} P_{L, U}=\gamma\left(\left(\operatorname{im} P_{L, U}\right)^{\perp}\right)$. Thus im $P_{L, U}$ is a Lagrangian (in particular closed) subspace of $U \cap \gamma U$.

From (6.29) it is now clear that im $P_{L, U}$ is isomorphic to $L \cap U / L \cap \operatorname{Ann}(U)$.

\section{Splittings of manifolds and the $\eta$-invariant II}

For the proof of Theorem 5.9, Lemma 5.2 was crucial. The proof of Lemma 5.2 depends on the Scott-Wojciechowski theorem 4.1. In this section we want to give proofs of Lemma 5.2 and Theorem 5.9 which are independent of the Scott-Wojciechowski theorem and which apply to all $P \in \operatorname{Gr}(A)$. We only use (a mild generalization of) Theorem 5.8. Moreover, we derive generalizations of two results due to L. Nicolaescu [26]. This in turn leads to a nicer version of the splitting formula for the $\eta$-invariant which involves our version of the Maslov triple index.

We first introduce a setting which slightly generalizes the one described in Section 2. Let $X$ be a compact Riemannian manifold with boundary $\partial X=Y \coprod Z$, i.e. the boundary is a disjoint union of two (not necessarily connected) manifolds. We assume

that in collars $U=U_{Y}$ and $U_{Z}$ we have $D=\gamma_{Y}\left(\frac{d}{d x}+A_{Y}\right)\left(\right.$ resp. $\left.D=\gamma_{Z}\left(\frac{d}{d x}+A_{Z}\right)\right)$ and that the $\pm i$-eigenspaces of $\gamma_{Y}\left(\gamma_{Z}\right)$ acting on $\operatorname{ker} A_{Y}\left(\operatorname{ker} A_{Z}\right)$ have the same dimension. 
The latter does not follow as in section 2; rather it is an assumption. We fix once and for all a $P_{Z} \in \operatorname{Gr}\left(A_{Z}\right)$. Then we can define the Calderón projector (relative to $\left.P_{Z}\right)$ in $\operatorname{Gr}\left(A_{Y}\right)$. Write again $A$ instead of $A_{Y}$. It will be convenient to address $Y, Z$ as boundary components although $Y, Z$ are not assumed to be connected.

The results of Sections 2 to 5 generalize verbatim to this more general setting. Also Theorem 5.8 applies to this setting since all proofs work locally in a collar of the separating hypersurface. The advantage of this setting is that it allows in particular to glue cylinders of the form $[0, \varepsilon] \times N$ with different boundary conditions on the ends to a manifold.

Lemma 7.1 (cf. [24, Lemma 2.5]). Let $M=[0, \varepsilon] \times N$ and $D=\gamma\left(\frac{d}{d x}+A\right)$ as before. Moreover, let $P, Q \in \operatorname{Gr}(A)$ and denote by $D_{P, Q}$ be the operator obtained by imposing the boundary condition $P$ at $\{0\} \times N$ and $I-Q$ at $\{\varepsilon\} \times N$. Then $\lambda \in \operatorname{spec} D_{P, Q}$ if and only if $-\lambda \in \operatorname{spec} D_{Q, P}$. In particular,

$$
\eta\left(D_{P, Q}\right)=-\eta\left(D_{Q, P}\right), \quad \operatorname{dim} \operatorname{ker} D_{P, Q}=\operatorname{dim} \operatorname{ker} D_{Q, P}
$$

PRoOF. The proof is exactly the same as the proof of [24, Lemma 2.5]. Namely, the isometry

$$
T: L^{2}\left([0, \varepsilon], L^{2}\left(E_{\mid N}\right)\right) \longrightarrow L^{2}\left([0, \varepsilon], L^{2}\left(E_{\mid N}\right)\right), \quad T f(x):=\gamma f(\varepsilon-x)
$$

maps the domain of $D_{P, Q}$ onto the domain of $D_{Q, P}$ and it anticommutes with $D$. Hence $T^{*} D_{P, Q} T=-D_{Q, P}$ and we are done.

Now let $M$ be a Riemannian manifold with boundary containing a separating hypersurface $N \subset(M \backslash \partial M)$. Let $D$ be a Dirac operator as in Section 5; i.e. in a collar neighborhood $[-\varepsilon, \varepsilon] \times N$ of $N, D$ has the form $D=\gamma\left(\frac{d}{d x}+A\right)$ as in (2.1). Moreover, we assume that the $\pm i$-eigenspaces of $\gamma$ acting on ker $A$ have the same dimension. Define $M^{\text {cut }}, M^{ \pm}$as in Section 5. We assume that on the boundary components of $\left(\partial M^{ \pm}\right) \backslash N$ self-adjoint boundary projections have been fixed once and for all.

Lemma 7.2. For any $P \in \operatorname{Gr}(A)$ we have $\widetilde{\eta}(D, M)-\widetilde{\eta}\left(D_{P}, M^{+}\right)-\widetilde{\eta}\left(D_{I-P}, M^{-}\right) \in \mathbb{Z}$.

Proof. Denote by $M_{\varepsilon}^{\text {cut }}$ the manifold with boundary obtained by removing $[-\varepsilon, \varepsilon] \times$ $N$ from $M$. As in Lemma 7.1 for $P, Q \in \operatorname{Gr}(A)$ we denote by $\eta\left(D_{P, Q},[-\varepsilon, \varepsilon] \times N\right)$ the $\eta$-invariant of the operator on $[-\varepsilon, \varepsilon] \times N$ obtained from $D$ by imposing the boundary condition $P$ at $\{-\varepsilon\} \times N$ and the boundary condition $I-Q$ at $\{\varepsilon\} \times N$. The $\bmod \mathbb{Z}$ gluing formula for the $\eta$-invariant (5.24) then implies

$$
\widetilde{\eta}(D, M) \equiv \widetilde{\eta}\left(D_{P^{+} \oplus\left(I-P^{+}\right)}, M_{\varepsilon}^{\text {cut }}\right)+\widetilde{\eta}\left(D_{P^{+}, P^{+}},[-\varepsilon, \varepsilon] \times N\right) \bmod \mathbb{Z}
$$

for $P^{+}=P^{+}(L)$ the Atiyah-Patodi-Singer projection with respect to a Lagrangian subspace $L \subset \operatorname{ker} A$. One easily checks that ker $D_{P^{+}, P^{+}}=\{0\}$, hence Lemma 7.1 implies

$$
\widetilde{\eta}\left(D_{P^{+}, P^{+}},[-\varepsilon, \varepsilon] \times N\right)=0 .
$$


Also by Lemma 7.1

$$
\begin{aligned}
\widetilde{\eta}\left(D_{P^{+}, P},\right. & {[-\varepsilon, 0] \times N)+\widetilde{\eta}\left(D_{P, P^{+}},[0, \varepsilon] \times N\right) } \\
= & \frac{1}{2} \operatorname{dim} \operatorname{ker}\left(D_{P^{+}, P},[-\varepsilon, 0] \times N\right)+\frac{1}{2} \operatorname{dim} \operatorname{ker}\left(D_{P, P^{+}},[0, \varepsilon] \times N\right) \\
= & \operatorname{dim} \operatorname{ker}\left(D_{P, P^{+}},[0, \varepsilon] \times N\right) \in \mathbb{Z} .
\end{aligned}
$$

Plugging this into (7.1) and applying again the $\bmod \mathbb{Z}$ splitting formula for the $\eta$ invariant we get

$$
\begin{aligned}
\widetilde{\eta}(D, M) & \equiv \widetilde{\eta}\left(D_{P^{+} \oplus\left(I-P^{+}\right)}, M_{\varepsilon}^{\text {cut }}\right)+\widetilde{\eta}\left(D_{P^{+}, P},[-\varepsilon, 0] \times N\right)+\widetilde{\eta}\left(D_{P, P^{+}},[0, \varepsilon] \times N\right) \\
& \equiv \widetilde{\eta}\left(D_{P}, M^{+}\right)+\widetilde{\eta}\left(D_{I-P}, M^{-}\right) \bmod \mathbb{Z} .
\end{aligned}
$$

Lemma 7.3. Lemma 5.2 holds for all $P_{0}, P_{1} \in \operatorname{Gr}(A)$.

Proof. We freely use the notations of Lemma 5.2 and its proof. By Lemma 7.2 we have for all $t$

$$
\begin{aligned}
& \widetilde{\eta}\left(D_{Q_{t}}, M^{\text {cut }}\right)-\widetilde{\eta}\left(D_{Q_{0}}, M^{\text {cut }}\right) \\
& =\left(\widetilde{\eta}\left(D_{Q_{t}}, M^{\text {cut }}\right)-\widetilde{\eta}(D, M)\right)-\left(\widetilde{\eta}\left(D_{Q_{0}}, M^{\text {cut }}\right)-\widetilde{\eta}(D, M)\right) \in \mathbb{Z} .
\end{aligned}
$$

Hence

$$
\frac{d}{d t} \widetilde{\eta}\left(D_{Q_{t}}, M^{\text {cut }}\right)=0
$$

and the assertion follows from Lemma 3.4 and Lemma 5.1.

Now we can prove the following considerable generalization of the splitting formula for the $\eta$-invariant. In Theorem 5.9 we assumed that $P \in \operatorname{Gr}_{\infty}(A)$. In the following theorem we only require $P \in \operatorname{Gr}(A)$.

THEOREM 7.4. The statement of Theorem 5.9 remains valid if $P \in \operatorname{Gr}(A)$ and $P_{t}$ is a smooth path in $\operatorname{Gr}(A)$ from $P$ to the Calderón projector.

Proof. The proof is exactly the same as the one of Theorem 5.9. One only has to invoke Lemma 7.3 instead of Lemma 5.2.

We next present generalizations of two results due to L. Nicolaescu [26].

THEOREM 7.5. Let $X$ be a manifold with boundary and $D(t), a \leq t \leq b$, a smooth family of Dirac operators. We assume that in a collar of the boundary $D$ takes the form $\gamma\left(\frac{d}{d x}+A(t)\right)$ as before. Let $P(t) \in \operatorname{Gr}(A(t))$ be a smooth family. Denote by $P_{X}(t)$ the Calderón projectors of $D(t)$, and $L_{X}(t)=\mathrm{im} P_{X}(t)$ the Cauchy data spaces. Then

$$
\mathrm{SF}\left(D_{P(t)}(t)\right)_{t \in[a, b]}=\operatorname{Mas}\left(P(t), P_{X}(t)\right)_{t \in[a, b]}=\operatorname{Mas}\left(\operatorname{ker} P(t), L_{X}(t)\right)_{t \in[a, b]} \cdot
$$

Note that $\gamma$ is assumed to be constant. This is essential. Note that in $\mathbf{1 4}$, Theorem 4.3] the collar of $\partial X$ was parametrized as $(-\varepsilon, 0] \times \partial X$. Their formula is obtained by invoking Corollary 6.7. 
Proof. We first consider the case $P(t) \in \operatorname{Gr}_{\infty}(A(t))$. Since $D_{P_{X}(t)}(t)$ is invertible, its spectral flow vanishes. We apply Lemma 3.4, Theorem 4.4, (6.5), and Theorem 6.6 to calculate

$$
\begin{aligned}
\mathrm{SF}\left(D_{P(t)}(t)\right)_{t \in[a, b]}= & \operatorname{SF}\left(D_{P(t)}(t)\right)_{t \in[a, b]}-\mathrm{SF}\left(D_{P_{X}(t)}(t)\right)_{t \in[a, b]} \\
= & \widetilde{\eta}\left(D_{P(b)}(b)\right)-\widetilde{\eta}\left(D_{P_{X}(b)}(b)\right)-\widetilde{\eta}\left(D_{P(a)}(a)\right)+\widetilde{\eta}\left(D_{P_{X}(a)}(a)\right) \\
& -\int_{a}^{b} \frac{d}{d t}\left(\widetilde{\eta}\left(D_{P(t)}(t)\right)-\widetilde{\eta}\left(D_{P_{X}(t)}(t)\right)\right) d t \\
= & \frac{1}{2 \pi i} \operatorname{tr} \log \left(\Phi(P(b)) \Phi\left(P_{X}(b)^{*}\right)\right)-\frac{1}{2 \pi i} \operatorname{tr} \log \left(\Phi(P(a)) \Phi\left(P_{X}(a)^{*}\right)\right) \\
& -\int_{a}^{b} \frac{1}{2 \pi i} \frac{d}{d t} \operatorname{tr} \log \left(\Phi(P(t)) \Phi\left(P_{X}(t)\right)^{*}\right) d t \\
= & -\operatorname{wind}\left(\Phi(P(t)) \Phi\left(P_{X}(t)\right)^{*}\right)_{t \in[a, b]}=\operatorname{Mas}\left(P(t), P_{X}(t)\right)_{t \in[a, b]} .
\end{aligned}
$$

Now suppose that $P(t)$ is arbitrary. Choose smooth paths $P_{0}(t)$ in $\operatorname{Gr}(A(0))$ and $P_{1}(t) \in \operatorname{Gr}(A(1))$ such that $P_{0}(0) \in \operatorname{Gr}_{\infty}(A(0)), P_{0}(1)=P(0), P_{1}(0)=P(1), P_{1}(1) \in$ $\operatorname{Gr}_{\infty}(A(1))$ and such that

$$
\operatorname{dim}\left(\operatorname{ker} P_{0}(t) \cap \operatorname{im} P_{X}(0)\right) \text { and } \operatorname{dim}\left(\operatorname{ker} P_{1}(t) \cap \operatorname{im} P_{X}(1)\right)
$$

are independent of $t$. The existence of $P_{0}, P_{1}$ follows from Lemma 6.1 by considering $\Phi(P(j)) \Phi\left(P_{X}(j)\right)^{*}, j=0,1$. In view of $(7.7)$ and Lemma 2.3 the dimension of the kernels of $D_{P_{0}(t)}(0)$ and $D_{P_{1}(t)}(1)$ are constant and hence the spectral flow of $D_{P_{0}(t)}(0)$ and $D_{P_{1}(t)}(1)$ vanishes. We may therefore compose the paths $D_{P_{0}(t)}(0), D_{P(t)}(t), D_{P_{1}}(t)$ without changing the spectral flow. Also $\operatorname{Mas}\left(P_{0}(t), P_{X}(0)\right)=\operatorname{Mas}\left(P_{1}(t), P_{X}(1)\right)=0$ in view of (7.7). In sum, without loss of generality we may assume that the family $P(t)$ satisfies $P(0) \in \operatorname{Gr}_{\infty}(A(0)), P(1) \in \mathrm{Gr}_{\infty}(A(1))$. Now consider the path $\Phi(P(t)) \Phi\left(P_{X}(t)\right)^{*}$ in $\mathscr{U}_{\text {Fred }}$. In view of Lemma 6.1 this path is homotopic rel endpoints to a path $f(t) \in \mathscr{U}_{\infty}$. Putting $\widetilde{P}(t):=\Phi^{-1}\left(f(t) \Phi\left(P_{X}(t)\right)\right) \in \mathrm{Gr}_{\infty}(A(t))$ we see that $\left(P(t), P_{X}(t)\right)$ is homotopic rel endpoints to the path $\left(\widetilde{P}(t), P_{X}(t)\right)$. Since homotopies with fixed endpoints neither change the spectral flow nor the Maslov index we find

$$
\begin{aligned}
\mathrm{SF}\left(D_{P(t)}(t)\right)_{t \in[a, b]} & =\mathrm{SF}\left(D_{\widetilde{P}(t)}(t)\right)_{t \in[a, b]}=\operatorname{Mas}\left(\widetilde{P}(t), P_{X}(t)\right)_{t \in[a, b]} \\
& =\operatorname{Mas}\left(P(t), P_{X}(t)\right)_{t \in[a, b]} .
\end{aligned}
$$

We also give a generalization of Nicolaescu's theorem for closed manifolds. The result in the following form was first proven in [13].

TheOREM 7.6. Let $M$ be a split manifold as in Section 5 and let $D(t), a \leq t \leq b$, be a smooth path of Dirac type operators such that in a collar of the separating hypersurface we have $D(t)=\gamma\left(\frac{d}{d x}+A(t)\right)$. Then

$$
\operatorname{SF}(D(t))_{t \in[a, b]}=\operatorname{Mas}_{\gamma}\left(P_{M^{-}}(t), I-P_{M^{+}}(t)\right)_{t \in[a, b]}=\operatorname{Mas}\left(L_{M^{-}}(t), L_{M^{+}}(t)\right)_{t \in[a, b]} .
$$

PROOF. Corollary 5.6 states that

$$
\mathrm{SF}(D(t))_{t \in[a, b]}=\mathrm{SF}\left(D_{I-P_{M^{+}}}(t)(t), M^{-}\right)_{t \in[a, b]} .
$$


$\eta$-INVARIANT, MASLOV INDEX, AND SPECTRAL FLOW

Applying Theorem 7.5 to the right hand side of (7.8) and using Corollary 6.7 yields

$$
\begin{aligned}
\operatorname{SF}\left(D_{I-P_{M^{+}}(t)}(t), M^{-}\right)_{t \in[a, b]} & =\operatorname{Mas}_{-\gamma}\left(I-P_{M^{+}}(t), P_{M^{-}}(t)\right)_{t \in[a, b]} \\
& =\operatorname{Mas}_{\gamma}\left(P_{M^{-}}(t), I-P_{M^{+}}(t)\right)_{t \in[a, b]},
\end{aligned}
$$

finishing the proof.

Notice that the proof of Theorem 7.6 does not rely on Theorem 5.9, and in particular does not use the result of $[7]$.

Finally, we state the following nicer version of the gluing formula for the $\eta$-invariant. We emphasize that the term $\tau_{\mu}\left(I-P_{M_{-}}, P, P_{M^{+}}\right)$, which was defined in Subsection 6.2, is an integer invariant which is defined completely in terms of the Hermitian symplectic structure on $L^{2}\left(E_{\mid N}\right)$.

Theorem 7.7. In the situation of Theorem 5.9, let $P \in \operatorname{Gr}(A)$. Then

$$
\widetilde{\eta}(D, M)=\widetilde{\eta}\left(D_{P}, M^{+}\right)+\widetilde{\eta}\left(D_{I-P}, M^{-}\right)-\tau_{\mu}\left(I-P_{M_{-}}, P, P_{M^{+}}\right) .
$$

Proof. We note again that $I-P_{M^{-}}-P_{M^{+}}$is trace class (cf. the proof of Theorem 5.10). Thus $I-P_{M^{-}}-P_{M^{+}}$is compact and hence the triple index $\tau_{\mu}\left(I-P_{M^{-}}, P, P_{M^{+}}\right)$ is well-defined for any $P \in \operatorname{Gr}(A)$.

Let $P_{t}, 0 \leq t \leq 1$, be a smooth path in $\operatorname{Gr}(A)$ from $P$ to the Calderón projector $P_{M^{+}}$. Notice that $\operatorname{Mas}_{\gamma}\left(I-P_{M^{-}}, P_{M^{+}}\right)=0$ since $I-P_{M^{-}}$and $P_{M^{+}}$are constant paths. From Theorem 7.4, Theorem 7.5, (6.21), Corollary 6.7, and Proposition 6.11 we infer

$$
\begin{aligned}
& \widetilde{\eta}(D, M)-\widetilde{\eta}\left(D_{P}, M^{+}\right)-\widetilde{\eta}\left(D_{I-P}, M^{-}\right) \\
& =\operatorname{SF}\left(D_{P_{t}}, M^{+}\right)_{t \in[0,1]}+\operatorname{SF}\left(D_{I-P_{t}}, M^{-}\right)_{t \in[0,1]} \\
& =\operatorname{Mas}_{\gamma}\left(P_{t}, P_{M^{+}}\right)_{t \in[0,1]}+\operatorname{Mas}_{-\gamma}\left(I-P_{t}, P_{M^{-}}\right)_{t \in[0,1]} \\
& =\operatorname{Mas}_{\gamma}\left(P_{t}, P_{M^{+}}\right)_{t \in[0,1]}+\operatorname{Mas}_{\gamma}\left(I-P_{M^{-}}, P_{t}\right)_{t \in[0,1]}-\operatorname{Mas}_{\gamma}\left(I-P_{M^{-}}, P_{M^{+}}\right)_{t \in[0,1]} \\
& =\tau_{\mu}\left(I-P_{M^{-}}, P_{M^{+}}, P_{M^{+}}\right)-\tau_{\mu}\left(I-P_{M_{-}}, P, P_{M^{+}}\right) \\
& =-\tau_{\mu}\left(I-P_{M_{-}}, P, P_{M^{+}}\right) .
\end{aligned}
$$

\section{Adiabatic stretching and applications to the Atiyah-Patodi-Singer $\rho$-invariant}

For the purpose of computation, one weakness of the splitting formulas of Theorems 5.9, 5.10, and 7.7 is that it is difficult in practice to identify the Calderón projector. In many applications it is more convenient to work with the Atiyah-Patodi-Singer projection $P^{+}(L)$, or at least some finite rank perturbation of $P^{+}(L)$, as a boundary condition. According to Theorem 5.9, this requires knowing the spectral flow of $D_{P_{t}}$ and $D_{I-P_{t}}$ along a path $P_{t}$ starting at the Calderón projector and ending at $P^{+}(L)$.

A natural choice of such a path is the path obtained by stretching the collar neighborhood of the separating surface. According to a theorem of Nicolaescu [26], the Calderón projector limits to a projection of the form $P_{>\nu}+\operatorname{proj}_{L}$, where $P_{>\nu}$ is the projection to the span of the eigenvectors of $A$ with eigenvalues greater than $\nu$ and 
$L$ is a Lagrangian subspace of the (finite-dimensional) span of eigenvectors of $A$ with eigenvalues in the range $[-\nu, \nu]$. The number $\nu$ is the non-resonance level [26] of $D$ acting on $M^{+}$and in particular is zero if and only if there are no $L^{2}$ solutions to $D \phi=0$ on the manifold obtained from $M^{+}$by adding an infinite collar. If $\nu=0$, then the limit of the Calderón projector is an Atiyah-Patodi-Singer projection $P^{+}(V)$ for a particular Lagrangian $V \subset \operatorname{ker} A$.

This approach works particularly well to study the odd signature operator and the Atiyah-Patodi-Singer $\rho_{\alpha}$ invariant [2], since the effect of the Riemannian metric is minimized in this important case. We present the details. The approach can be made to work for arbitrary Dirac operators as well, however additional correction terms appear corresponding to the 1-parameter family of operators acting on $M$ and $M^{ \pm}$as the collar of the separating hypersurface is stretched to infinity. We will make some comments about the case of general Dirac operators at the end of this section.

8.1. The odd signature operator. Let $X$ be a compact manifold of dimension $2 n+1$, with (possibly empty) boundary $\partial X^{2 n}$. Assume a collar of $\partial X$ is isometric to $[0, \varepsilon) \times \partial X$. Let $\alpha: \pi_{1}(X) \rightarrow U(n)$ be a representation. To $\alpha$ one can assign a flat vector bundle, that is, a $\mathbb{C}^{n}$ bundle $E \rightarrow X$ together with a flat connection $B$ on $E$ so that the holonomy representation of $B$ is equal to $\alpha$. If $\partial X$ is non-empty, we may assume, by gauge transforming $B$ if necessary, that $B$ is in temporal gauge on the collar, in other words there is a flat $U(n)$ connection $b$ on $E_{\mid \partial X}$ so that the restriction of $B$ to the collar $[0, \varepsilon) \times \partial X$ is of the form

$$
B_{[0, \varepsilon) \times \partial X}=q^{*}(b),
$$

where $q:[0, \varepsilon) \times \partial X \rightarrow \partial X$ is the projection to the second factor.

Let $d_{B}: \Omega^{p}(X ; E) \rightarrow \Omega^{p+1}(X ; E)$ and $d_{b}: \Omega^{p}\left(\partial X ; E_{\mid \partial X}\right) \rightarrow \Omega^{p+1}\left(\partial X ; E_{\mid \partial X}\right)$ denote the associated coupled DeRham operators. Note that $d_{B}^{2}$ and $d_{b}^{2}$ are zero since $B$ and $b$ are flat. The cohomology of the complex $\left(\Omega^{*}(X ; E), d_{B}\right)$ (resp. $\left.\left(\Omega^{*}\left(\partial X ; E_{\mid \partial X}\right), d_{b}\right)\right)$ is identified via the DeRham theorem with the singular cohomology $H^{*}\left(X ; \mathbb{C}_{\alpha}^{n}\right)$ (resp. $\left.H^{*}\left(\partial X ; \mathbb{C}_{\alpha}^{n}\right)\right)$, where $\mathbb{C}_{\alpha}^{n}$ denotes the local coefficient system determined by the representation $\alpha$.

The odd signature operator on $X$ coupled to the flat connection $B$ is the operator

$$
D_{B}: \underset{p}{\oplus} \Omega^{2 p}(X ; E) \rightarrow \underset{p}{\oplus} \Omega^{2 p}(X ; E)
$$

defined by

$$
D_{B}(\beta)=i^{n+1}(-1)^{p-1}\left(* d_{B}-d_{B} *\right)(\beta) \text { for } \beta \in \Omega^{2 p}(X ; E),
$$

where $*: \Omega^{k}(X ; E) \rightarrow \Omega^{2 n+1-k}(X ; E)$ denotes the Hodge $*$ operator (see [2]).

The operator $D_{B}$ is a symmetric Dirac operator. Its square is the twisted Laplacian acting on even bundle-valued forms:

$$
D_{B}^{2}=d_{B}^{*} d_{B}+d_{B} d_{B}^{*}
$$


In particular $D_{B}$ is self-adjoint if $X$ has empty boundary and in that case its kernel can be identified with the twisted cohomology group $\oplus_{p} H^{2 p}\left(X ; \mathbb{C}_{\alpha}^{n}\right)$ by the Hodge and DeRham theorems. This implies that the dimension of the kernel of $D_{B}$ is independent of the choice of Riemannian metric if $X$ is closed.

Define a restriction map

$$
r: \underset{p}{\oplus} \Omega^{2 p}(X ; E) \rightarrow \underset{k}{\oplus} \Omega^{k}\left(\partial X ; E_{\mid \partial X}\right)
$$

by the formula

$$
r(\beta)=i^{*}(\beta)+i^{*}(* \beta)
$$

where $i: \partial X \hookrightarrow X$ denotes the inclusion of the boundary.

To avoid confusion we denote the Hodge $*$ operator on the boundary by $\hat{*}$, thus

$$
\hat{*}: \Omega^{k}\left(\partial X ; E_{\mid \partial X}\right) \rightarrow \Omega^{2 n-k}\left(\partial X ; E_{\mid \partial X}\right) .
$$

We use $\hat{*}$ to define

$$
\gamma: \underset{k}{\oplus} \Omega^{k}\left(\partial X ; E_{\mid \partial X}\right) \rightarrow \underset{k}{\oplus} \Omega^{k}\left(\partial X ; E_{\mid \partial X}\right)
$$

by

$$
\gamma(\beta)= \begin{cases}i^{n+1}(-1)^{p-1} \hat{*} \beta & \text { if } \beta \in \Omega^{2 p}\left(\partial X ; E_{\mid \partial X}\right), \\ i^{n+1}(-1)^{n-q} \hat{*} \beta & \text { if } \beta \in \Omega^{2 q+1}\left(\partial X ; E_{\mid \partial X}\right) .\end{cases}
$$

Finally, we define the operator

$$
A_{b}: \underset{k}{\oplus} \Omega^{k}\left(\partial X ; E_{\mid \partial X}\right) \rightarrow \underset{k}{\oplus} \Omega^{k}\left(\partial X ; E_{\mid \partial X}\right)
$$

by

$$
A_{b}(\beta)=\left\{\begin{aligned}
-\left(d_{b} \hat{*}+\hat{*} d_{b}\right) \beta & \text { if } \beta \in \oplus_{k} \Omega^{2 k}\left(\partial X ; E_{\mid \partial X}\right), \\
\left(d_{b} \hat{*}+\hat{*} d_{b}\right) \beta & \text { if } \beta \in \oplus_{k} \Omega^{2 k+1}\left(\partial X ; E_{\mid \partial X}\right) .
\end{aligned}\right.
$$

The following facts are routine to verify.

1. $A_{b}$ is a self-adjoint Dirac operator on $\partial X$.

2. $r$ induces an identification $\Phi: \oplus_{p} \Omega^{2 p}([0, \varepsilon) \times \partial X ; E) \rightarrow C^{\infty}\left([0, \varepsilon), \oplus_{k} \Omega^{k}(\partial X ; E)\right)$ which is isometric with respect to the $L^{2}$-structures. Moreover,

$$
\Phi D_{B} \Phi^{*}=\gamma\left(\frac{\partial}{\partial x}+A_{b}\right)
$$

where $x$ denotes the collar coordinate.

3. $\gamma A_{b}=-A_{b} \gamma$.

4. $\gamma^{2}=-I$.

5. $A_{b}$ reverses the parity of forms.

6. $A_{b} d_{b}=-d_{b} A_{b}$ and $A_{b} d_{b}^{*}=-d_{b}^{*} A_{b}$, where $d_{b}^{*}=-\hat{*} d_{b} \hat{*}$ is the $L^{2}$-adjoint of $d_{b}$.

7. $A_{b}^{2}$ preserves the subspace $\Omega^{k}\left(\partial X ; E_{\mid \partial X}\right)$ for each $k$, and equals the twisted Laplacian on $k$-forms, $A_{b}^{2}=\Delta_{b}=d_{b} d_{b}^{*}+d_{b}^{*} d_{b}$.

8. The kernel of $A_{b}$ equals ker $A_{b}^{2}=\operatorname{ker} \Delta_{b}$, which is identified using the Hodge theorem with the DeRham cohomology of the complex $\left(\Omega^{k}\left(\partial X ; E_{\mid \partial X}\right), d_{b}\right)$. The DeRham isomorphism identifies the DeRham cohomology with twisted cohomology $H^{*}\left(\partial X ; \mathbb{C}_{\alpha}^{n}\right)$, where $\alpha: \pi_{1} \partial X \rightarrow U(n) \subset G L\left(\mathbb{C}^{n}\right)$ is the holonomy representation of the flat connection $b$. 
The first 5 facts do not depend on $B$ being a flat connection, and hold for any $U(n)$ connection in temporal gauge near the boundary. The last three depend on $b$ being flat.

For convenience we simplify the notation as follows. Let $\Omega_{X}^{\text {even }}$ denote $\oplus_{p} \Omega^{2 p}(X ; E)$ and let $\Omega_{\partial X}^{*}$ denote $\oplus_{k} \Omega^{k}\left(\partial X ; E_{\mid \partial X}\right)$. The $L^{2}$ completion of $\Omega_{\partial X}^{*}$ will be denoted by $L^{2}\left(\Omega_{\partial X}^{*}\right)$. We will often drop the subscripts " $B$ " and " $b$ " and, for example, write $D$ for $D_{B}, A$ for $A_{b}$, and $d$ for $d_{B}$ or $d_{b}$.

The self-adjoint operator $A$ induces a spectral decomposition of $L^{2}\left(\Omega_{\partial X}^{*}\right)$. We denote the $\mu$-eigenspace of $A$ by $E_{\mu}$. Given $\nu \geq 0$ we will also use the notation

$$
\begin{gathered}
F_{\nu}^{+}=\operatorname{span}_{L^{2}}\left\{\psi_{\mu} \mid A \psi_{\mu}=\mu \psi_{\mu}, \mu>\nu\right\}=\underset{\mu>\nu}{\oplus} E_{\mu}, \\
F_{\nu}^{-}=\operatorname{span}_{L^{2}}\left\{\psi_{\mu} \mid A \psi_{\mu}=\mu \psi_{\mu}, \mu<-\nu\right\}=\underset{\mu<-\nu}{\oplus} E_{\mu}, \\
E_{\nu}^{+}=\underset{0<\mu \leq \nu}{\bigoplus} E_{\mu},
\end{gathered}
$$

and

$$
E_{\nu}^{-}=\underset{-\nu \leq \mu<0}{\bigoplus} E_{\mu}
$$

Thus $E_{\nu}^{-}$is the finite-dimensional span of the eigenvectors of $A$ with eigenvalues $\mu$ in the range $-\nu \leq \mu<0, E_{\nu}^{+}$corresponds to the range $0<\mu \leq \nu$ (if $\nu=0$, then $\left.E_{\nu}^{ \pm}=0\right)$. Similarly $F_{\nu}^{-}$is the infinite-dimensional space spanned by eigenvectors with eigenvalues $\mu$ satisfying $\mu<-\nu$, and $F_{\nu}^{+}$corresponds to $\mu>\nu$. In particular $F_{0}^{+}$denotes the positive eigenspan and $F_{0}^{-}$the negative eigenspan of $A$. This gives an orthogonal decomposition

$$
L^{2}\left(\Omega_{\partial X}^{*}\right)=F_{\nu}^{-} \oplus E_{\nu}^{-} \oplus \operatorname{ker} A \oplus E_{\nu}^{+} \oplus F_{\nu}^{+} .
$$

Another orthogonal decomposition of $L^{2}\left(\Omega_{\partial X}^{*}\right)$ is the Hodge decomposition:

$$
L^{2}\left(\Omega_{\partial X}^{*}\right)=\operatorname{im} d \oplus \operatorname{ker} A \oplus \operatorname{im} d^{*} .
$$

We introduce a notational convention: the decomposition 8.2 is compatible with the operators $d, d^{*}$ in the sense that we have decompositions of domains:

$$
\begin{aligned}
\mathscr{D}(d) & =\left(F_{\nu}^{-} \cap \mathscr{D}(d)\right) \oplus E_{\nu}^{-} \oplus \operatorname{ker} A \oplus E_{\nu}^{+} \oplus\left(F_{\nu}^{+} \cap \mathscr{D}(d)\right), \\
\mathscr{D}\left(d^{*}\right) & =\left(F_{\nu}^{-} \cap \mathscr{D}\left(d^{*}\right)\right) \oplus E_{\nu}^{-} \oplus \operatorname{ker} A \oplus E_{\nu}^{+} \oplus\left(F_{\nu}^{+} \cap \mathscr{D}\left(d^{*}\right) .\right.
\end{aligned}
$$

Note that $E_{\nu}^{-} \oplus \operatorname{ker} A \oplus E_{\nu}^{+}$consists of smooth sections hence $\left(E_{\nu}^{-} \oplus \operatorname{ker} A \oplus E_{\nu}^{+}\right) \cap$ $\mathscr{D}(d) \cap \mathscr{D}\left(d^{*}\right)=E_{\nu}^{-} \oplus \operatorname{ker} A \oplus E_{\nu}^{+}$. By slight abuse of notation we will write in the sequel $d^{(*)}\left(F_{\nu}^{ \pm}\right)$for the image of $d^{(*)}$ on $F_{\nu}^{ \pm} \cap \mathscr{D}\left(d^{(*)}\right)$.

The relations between the decompositions (8.2) and (8.3) are summarized in the following useful lemma.

LEMMA 8.1.

1. $d\left(F_{\nu}^{ \pm}\right) \subset F_{\nu}^{\mp}$ and $d^{*}\left(F_{\nu}^{ \pm}\right) \subset F_{\nu}^{\mp}$.

2. $F_{\nu}^{+}=d\left(F_{\nu}^{-}\right) \oplus d^{*}\left(F_{\nu}^{-}\right)=\left(\operatorname{ker} d: F_{\nu}^{+} \rightarrow F_{\nu}^{-}\right) \oplus\left(\operatorname{ker} d^{*}: F_{\nu}^{+} \rightarrow F_{\nu}^{-}\right)$.

3. $F_{\nu}^{-}=d\left(F_{\nu}^{+}\right) \oplus d^{*}\left(F_{\nu}^{+}\right)=\left(\operatorname{ker} d: F_{\nu}^{-} \rightarrow F_{\nu}^{+}\right) \oplus\left(\operatorname{ker} d^{*}: F_{\nu}^{-} \rightarrow F_{\nu}^{+}\right)$.

4. $d\left(E_{-\mu}\right) \subset E_{\mu}$ and $d^{*}\left(E_{-\mu}\right) \subset E_{\mu}$, and for $\mu \neq 0, E_{\mu}=d\left(E_{-\mu}\right) \oplus d^{*}\left(E_{-\mu}\right)$.

5. $\gamma(\operatorname{ker} d)=\operatorname{ker} d^{*}$ and $\gamma\left(\operatorname{ker} d^{*}\right)=\operatorname{ker} d$. 
Proof. If $A \beta=\mu \beta$, then $A d \beta=-d A \beta=-\mu d \beta$, and similarly $A d^{*} \beta=-\mu d^{*} \beta$. This proves the first assertion and the first part of the fourth assertion.

If $\beta \in F_{\nu}^{+}$, then $\beta$ is orthogonal to ker $A$, since the decomposition (8.2) is orthogonal. Since the decomposition (8.3) is also orthogonal, $\beta$ has the orthogonal decomposition $\beta=d \tau+d^{*} \sigma$. Write $\tau=\tau_{-}+\tau_{+} \in F_{\nu}^{-} \oplus F_{\nu}^{+}$, and similarly $\sigma=\sigma_{-}+\sigma_{+}$. Then

$$
\beta=d \tau_{-}+d \tau_{+}+d^{*} \sigma_{-}+d^{*} \sigma_{+} .
$$

Since $\beta \in F_{\nu}^{+}$, the first assertion implies that $d \tau_{+}=0=d^{*} \sigma_{+}$, so that $\beta=d \tau_{-}+d^{*} \sigma_{-}$. The second assertion follows from this and the consequence $d\left(F_{\nu}^{-} \oplus F_{\nu}^{+}\right)=$ker $d$ : $F_{\nu}^{-} \oplus F_{\nu}^{+} \rightarrow F_{\nu}^{-} \oplus F_{\nu}^{+}$of the DeRham theorem. The third assertion is proved similarly, as is the second part of the fourth assertion.

The last assertion follows from the identity $d^{*}=-\hat{*} d \hat{*}$ and the fact that $\gamma$ equals $\hat{*}$ up to a non-zero constant.

Of particular concern will be the symplectic structure on ker $A$. The isomorphism $\gamma$ preserves ker $A$, satisfies $\gamma^{2}=-I$, and acts with signature zero, since $(\partial X, A)$ bounds $(X, D)$. Therefore ker $A$ is a finite-dimensional Hermitian symplectic subspace of $L^{2}\left(\Omega_{\partial X}^{*}\right)$.

Notice that the restrictions of $\langle\rangle,, \gamma$, and $\omega$ to $\operatorname{ker} A$ induce these structures on the cohomology $H^{*}\left(\partial X ; \mathbb{C}_{\alpha}^{n}\right)$ via the Hodge and DeRham isomorphisms. The inner product $\langle$,$\rangle and complex structure \gamma$ depend on the choice of Riemannian metric on $\partial X$, but the symplectic structure $\omega$ does not: if $\beta_{1} \in \operatorname{ker} A$ is a $p$-form and $\beta_{2} \in \operatorname{ker} A$ is a $2 n-p$ form, then

$$
\omega\left(\beta_{1}, \beta_{2}\right)=\left\langle\beta_{1}, \gamma\left(\beta_{2}\right)\right\rangle=i^{r} \int_{\partial X} \beta_{1} \wedge \beta_{2}
$$

where the constant $i^{r}$ depends only on $p$ and $n$ (and we have suppressed the notation for the inner product in the flat $\mathbb{C}^{n}$ bundle $E_{\mid \partial X}$ ). Since wedge products and cup products correspond via the DeRham isomorphism, $\omega$ coincides with the cup product up to a power of $i$, and in particular is a homotopy invariant. To put this differently, The cup product, together with the standard $U(n)$-invariant Hermitian inner product on $\mathbb{C}^{n}$, induces a skew-hermitian form

$$
\omega: H^{*}\left(\partial X ; \mathbb{C}^{n}\right) \times H^{*}\left(\partial X ; \mathbb{C}^{n}\right) \rightarrow \mathbb{C}, \omega\left(\beta_{1}, \beta_{2}\right)=i^{r}\left(\beta_{1} \cup \beta_{2}\right) \cap[\partial X]
$$

which is a homotopy invariant of the pair $\left(\partial X, \alpha_{\mid \partial X}\right)$. Fixing a Riemannian metric on $\partial X$ induces a positive definite Hermitian inner product and an isomorphism $\gamma$ on ker $A$. The Hodge and DeRham theorems define an isomorphism ker $A \rightarrow H^{*}\left(\partial X ; \mathbb{C}_{\alpha}^{n}\right)$ which takes the form $\langle x, \gamma(y)\rangle$ to the form $\omega(x, y)$.

The following lemma collects some useful information about symplectic subspaces and symplectic reduction. For more details about symplectic reduction in this setting the reader should consult Section 6.3 and [26].

LEMMA 8.2.

1. Let $S \subset L^{2}\left(\Omega_{\partial X}^{*}\right)$ be a closed subspace satisfying $\gamma(S) \perp S$. Then $S \oplus \gamma(S)$ is a Hermitian symplectic subspace of $L^{2}\left(\Omega_{\partial X}^{*}\right)$, and $S$ is a Lagrangian subspace of $S \oplus \gamma(S)$. 
2. If $\nu \geq 0$, then $F_{\nu}^{-} \oplus F_{\nu}^{+}, E_{\nu}^{-} \oplus E_{\nu}^{+}, E_{\nu}^{-} \oplus \operatorname{ker} A \oplus E_{\nu}^{+}$, and $d\left(E_{\nu}^{ \pm}\right) \oplus d^{*}\left(E_{\nu}^{\mp}\right)$ are Hermitian symplectic subspaces of $L^{2}\left(\Omega_{\partial X}^{*}\right)$.

3. Given a Lagrangian subspace $L \subset L^{2}\left(\Omega_{\partial X}^{*}\right)$ so that $\left(L, F_{0}^{-}\right)$form a Fredholm pair of subspaces, then

$$
R_{\nu}(L):=\frac{L \cap\left(F_{\nu}^{-} \oplus E_{\nu}^{-} \oplus \operatorname{ker} A \oplus E_{\nu}^{+}\right)}{L \cap F_{\nu}^{-}} \subset E_{\nu}^{-} \oplus \operatorname{ker} A \oplus E_{\nu}^{+}
$$

is a Lagrangian subspace, called the symplectic reduction of $L$ with respect to $F_{\nu}^{-}$.

Proof. 1. Notice that $\gamma$ preserves $S \oplus \gamma(S)$. Let $K_{ \pm i}$ denote the $\pm i$ eigenspaces of $\gamma$ acting on $S \oplus \gamma(S)$. It is easy to check that since $\gamma(S)$ is orthogonal to $S$, the projections $S \oplus \gamma(S) \rightarrow K_{ \pm i}$ restrict to isomorphisms on $S$. Thus the $\pm i$ eigenspaces of $\gamma$ on $S \oplus \gamma(S)$ have the same dimension (or are both infinite). This shows that $S \oplus \gamma(S)$ is a symplectic subspace of $L^{2}\left(\Omega_{\partial X}^{*}\right)$. Clearly $S$ is a Lagrangian subspace of $S \oplus \gamma(S)$.

2. For $F_{\nu}^{-} \oplus F_{\nu}^{+}$, take $S=F_{\nu}^{-}$and apply the first assertion. For $E_{\nu}^{-} \oplus E_{\nu}^{+}$, take $S=E_{\nu}^{-}$. For $d\left(E_{\nu}^{ \pm}\right) \oplus d^{*}\left(E_{\nu}^{\mp}\right)$, take $S=d\left(E_{\nu}^{ \pm}\right)$; then $\gamma(S)=\hat{*} S=\hat{*} d\left(E_{\nu}^{ \pm}\right)=$ $\hat{*} d\left(\hat{*} E_{\nu}^{\mp}\right)=d^{*}\left(E_{\nu}^{\mp}\right)$. That $\operatorname{ker} A$ is symplectic was discussed above; hence the direct sum $E_{\nu}^{-} \oplus \operatorname{ker} A \oplus E_{\nu}^{+}$is symplectic.

3. We apply Proposition 6.12 with $U=F_{\nu}^{-} \oplus E_{\nu}^{-} \oplus \operatorname{ker} A \oplus E_{\nu}^{+}$. We have $\operatorname{Ann}(U)=$ $F_{\nu}^{-}$and $U \cap \gamma U=E_{\nu}^{-} \oplus \operatorname{ker} A \oplus E_{\nu}^{+}$. Since $\left(L, F_{0}^{-}\right)$form a Fredholm pair and $F_{0}^{-} / F_{\nu}^{-}$is finite-dimensional, also $(L, \operatorname{Ann}(U))=\left(L, F_{\nu}^{-}\right)$is Fredholm. Consequently $L+\operatorname{Ann}(U)$ is closed and we reach the desired conclusion using Proposition 6.12.

In preparation for what follows we define the following enlargements of $X$. Given $r \geq 0$ define

$$
X_{r}=([-r, 0] \times \partial X) \cup X
$$

and

$$
X_{\infty}=((-\infty, 0] \times \partial X) \cup X .
$$

Thus $X_{r}$ has a collar of length $r$ attached to $X$ and $X_{\infty}$ is obtained from $X$ by attaching an infinitely long collar. Equation (8.1) can be used on the collar to define a natural extension of $D$ to $X_{r}$ and $X_{\infty}$.

The key to identifying the adiabatic limit of the Calderón projector is the following result.

Proposition 8.3. Suppose that the boundary of $X$ is non-empty, and suppose that $\beta \in \Omega_{X}^{\text {even }}$ satisfies $D \beta=0$ and $r(\beta) \in F_{0}^{-} \oplus \operatorname{ker} A=\operatorname{span}\left\{\psi_{\mu} \mid \mu \leq 0\right\}$. Then $d \beta=0$, $d(* \beta)=0$, and $d(r(\beta))=0$.

Proof. Naturality of the exterior derivative implies that $d\left(i^{*}(z)\right)=i^{*}(d z)$ for any $z \in \Omega_{X}^{k}$. It suffices, therefore, to show that $d \beta=0$ and $d(* \beta)=0$, since $r(\beta)=$ $i^{*}(\beta)+i^{*}(* \beta)$ and hence

$$
d(r(\beta))=d\left(i^{*}(\beta)+i^{*}(* \beta)\right)=i^{*}(d \beta+d * \beta)=0 .
$$


Following [1], since $D \beta=0$ and $r(\beta) \in F_{0}^{-} \oplus \operatorname{ker} A, \beta$ has a Fourier expansion on the collar $[0, \varepsilon) \times \partial X$ of the form

$$
\beta_{\mid[0, \varepsilon) \times \partial X}=\sum_{\mu<0} c_{\mu} e^{-x \mu} \psi_{\mu}+k,
$$

where $k \in \operatorname{ker} A, x \in[0, \varepsilon)$, and $\psi_{\mu} \in E_{\mu}$. Equation 8.7 can be used to extend $\beta$ to a bounded form on $X_{\infty}$ so that the extension still satisfies $D \beta=0$.

Notice that $d k=0$ since $k \in \operatorname{ker} A$ and $k$ is independent of the collar parameter. Thus $d \beta$ decays exponentially on the infinite collar $(-\infty, 0] \times \partial X$. Write $\beta=\sum \beta_{2 p}$. Then

$$
\begin{aligned}
\left\langle d * \beta_{2 p}, * d \beta_{2(p-1)}\right\rangle_{L^{2}\left(\Omega_{X_{r}}^{*}\right)} & = \pm \int_{X_{r}} d * \beta_{2 p} \wedge \beta_{2(p-1)} \\
& = \pm \int_{X_{r}} d\left(* \beta_{2 p} \wedge d \beta_{2(p-1)}\right) \\
& = \pm \int_{\partial X \times\{-r\}} i^{*}\left(* \beta_{2 p}\right) \wedge i^{*}\left(d \beta_{2(p-1)}\right) .
\end{aligned}
$$

The last step follows from Stokes's theorem. As $r$ increases to infinity, the last integral converges to zero since $* \beta_{2 p}$ is bounded on $X_{\infty}$ and $d \beta_{2(p-1)}$ exponentially decays. It follows that $d * \beta_{2 p}$ and $* d \beta_{2(p-1)}$ are orthogonal in $L^{2}\left(\Omega_{X_{\infty}}^{2(n-p-1)}\right)$. Now

$$
0=D \beta=i^{n+1} \sum_{p}(-1)^{p}\left(d * \beta_{2 p}+* d \beta_{2(p-1)}\right),
$$

with this sum expressed as a sum of homogeneous components. Thus $d * \beta_{2 p}$ and $* d \beta_{2(p-1)}$ both vanish for each $p$, and therefore $d * \beta$ and $* d \beta$ both vanish.

As an application of Proposition 8.3 we can identify the limiting values of extended $L^{2}$ solutions of $D \beta=0$ in the sense of [1]. Recall that this is the subspace of $\operatorname{ker} A$ defined by

$$
V_{\alpha}=\left\{\begin{array}{l|l}
k \mid \begin{array}{l}
\text { there exists a } \beta \in \Omega_{X}^{\text {even }} \text { with } D \beta=0 \\
\text { and } r(\beta)=f_{-}+k \in F_{0}^{-} \oplus \operatorname{ker} A
\end{array}
\end{array}\right\}
$$

The terminology is justified by the Fourier expansion (8.7). In light of the unique continuation property for $D$ (which says that for each $\ell \in L_{X}$ there exists a unique $\beta$ with $D \beta=0$ and $r(\beta)=\ell$ ), it is easy to see that $V_{\alpha}$ has the alternative description as a symplectic reduction:

$$
V_{\alpha}=R_{0}\left(L_{X}\right)=\frac{L_{X} \cap\left(F_{0}^{-} \oplus \operatorname{ker} A\right)}{L_{X} \cap F_{0}^{-}} \subset \operatorname{ker} A .
$$

Equation (8.9) says that $V_{\alpha}$ is the symplectic reduction of the Cauchy data space $L_{X}$ with respect to subspace $F_{0}^{-}$. Using Lemma 8.2 it follows that $V_{\alpha}$ is a Lagrangian subspace of $\operatorname{ker} A$. 
The kernel of $A$ is identified via the Hodge and DeRham theorems with the cohomology $H^{*}\left(\partial X, \mathbb{C}_{\alpha}^{n}\right)$. The next result identifies $V_{\alpha}$.

Corollary 8.4. The space $V_{\alpha}$ of limiting values of extended $L^{2}$ solutions to $D \beta=0$ on $X_{\infty}$ is identified via the Hodge and DeRham theorems with the image of the cohomology of $X$ in the cohomology of $\partial X$ (with local coefficients in the corresponding flat $\mathbb{C}^{n}$ bundle):

$$
V_{\alpha}=\operatorname{im} i^{*}: H^{*}\left(X ; \mathbb{C}_{\alpha}^{n}\right) \rightarrow H^{*}\left(\partial X ; \mathbb{C}_{\alpha}^{n}\right)
$$

Proof. Proposition 8.3 shows that if $\beta \in \Omega_{X}^{\text {even }}$ satisfies $D \beta=0$ and $r(\beta) \in$ $F_{0}^{-} \oplus \operatorname{ker} A$, then $\beta$ and $* \beta$ are closed forms. Thus they represent classes in $H^{*}\left(X ; \mathbb{C}_{\alpha}^{n}\right)$. Since $r(\beta)=i^{*}(\beta)+i^{*}(* \beta)$, it follows that $r(\beta)$ is a closed form on $\partial X$ representing a class in im $i^{*}: H^{*}\left(X ; \mathbb{C}_{\alpha}^{n}\right) \rightarrow H^{*}\left(\partial X ; \mathbb{C}_{\alpha}^{n}\right)$. The identification of cohomology with harmonic forms takes $[r(\beta)]=\left[f_{-}+k\right]$ to $k$ and so

$$
V_{\alpha} \subset \operatorname{im} i^{*}: H^{*}\left(X ; \mathbb{C}_{\alpha}^{n}\right) \rightarrow H^{*}\left(\partial X ; \mathbb{C}_{\alpha}^{n}\right) .
$$

The space $V_{\alpha}$ is a Lagrangian subspace, as is $\operatorname{im} i^{*}: H^{*}\left(X ; \mathbb{C}_{\alpha}^{n}\right) \rightarrow H^{*}\left(\partial X ; \mathbb{C}_{\alpha}^{n}\right)$ by a standard argument using Poincaré duality. Since any two Lagrangian subspaces of a finite-dimensional symplectic vector space have the same dimension, $V_{\alpha}=\operatorname{im} i^{*}$.

It follows from Lemma 8.1 that $E_{\nu}^{ \pm}=d\left(E_{\nu}^{\mp}\right) \oplus d^{*}\left(E_{\nu}^{\mp}\right)$, and so the decomposition 8.2 can be refined to

$$
L^{2}\left(\Omega_{\partial X}^{*}\right)=F_{\nu}^{-} \oplus d\left(E_{\nu}^{+}\right) \oplus d^{*}\left(E_{\nu}^{+}\right) \oplus \operatorname{ker} A \oplus d\left(E_{\nu}^{-}\right) \oplus d^{*}\left(E_{\nu}^{-}\right) \oplus F_{\nu}^{+} .
$$

The terms in this decomposition are arranged according to increasing eigenvalues. We will find it convenient to rewrite this in a different order, as a symplectic direct sum of symplectic subspaces:

$$
L^{2}\left(\Omega_{\partial X}^{*}\right)=\left(F_{\nu}^{-} \oplus F_{\nu}^{+}\right) \oplus\left(d\left(E_{\nu}^{+}\right) \oplus d^{*}\left(E_{\nu}^{-}\right)\right) \oplus\left(d^{*}\left(E_{\nu}^{+}\right) \oplus d\left(E_{\nu}^{-}\right)\right) \oplus \operatorname{ker} A .
$$

We will refer to the decomposition (8.11) frequently. Notice that $F_{\nu}^{-} \oplus F_{\nu}^{+}$is infinitedimensional and the other three symplectic summands in this decomposition have finite dimension.

There exists a $\nu \geq 0$ so that the Cauchy data space $L_{X}$ of $D$ is transverse to $F_{\nu}^{-}$. This is because $L_{X} \cap F_{0}^{-}$is finite-dimensional, and as $\nu$ increases, $L_{X} \cap F_{\nu}^{-}$decreases to zero. Nicolaescu calls the smallest such $\nu$ the non-resonance level for $D$.

We can now state and prove a theorem identifying the limit of the Calderón projectors of $D$ acting on $X_{r}$ as $r$ goes to infinity. Denote by $L_{X}^{r}$ the Cauchy data space (i.e. the image of the Calderón projector) of $D$ acting on $X_{r}=([-r, 0] \times \partial X) \cup X$.

THEOREM 8.5. Let $X$ be an odd-dimensional manifold with boundary and $D$ the odd signature operator coupled to a flat connection $B$ acting on $X$ as above. Let $\nu \geq 0$ be any number greater than or equal to the non-resonance level for $D$.

Then there exists a subspace

$$
W_{\alpha} \subset d\left(E_{\nu}^{+}\right) \subset F_{0}^{-}
$$


isomorphic to the image of

$$
H^{\text {even }}\left(X, \partial X ; \mathbb{C}_{\alpha}^{n}\right) \rightarrow H^{\text {even }}\left(X ; \mathbb{C}_{\alpha}^{n}\right)
$$

so that if $W_{\alpha}^{\perp}$ denotes the orthogonal complement of $W_{\alpha}$ in $d\left(E_{\nu}^{+}\right)$, then with respect to the decomposition (8.11) of $L^{2}\left(\Omega_{\partial X}^{*}\right)$ into symplectic subspaces, the adiabatic limit of the Cauchy data spaces decomposes as a direct sum of Lagrangian subspaces:

$$
\lim _{r \rightarrow \infty} L_{X}^{r}=F_{\nu}^{+} \oplus\left(W_{\alpha} \oplus \gamma\left(W_{\alpha}^{\perp}\right)\right) \oplus d\left(E_{\nu}^{-}\right) \oplus V_{\alpha} .
$$

where $V_{\alpha} \subset \operatorname{ker} A=H^{*}\left(\partial X ; \mathbb{C}_{\alpha}^{n}\right)$ denotes the image of $H^{*}\left(X ; \mathbb{C}_{\alpha}^{n}\right) \rightarrow H^{*}\left(\partial X ; \mathbb{C}_{\alpha}^{n}\right)$.

Proof. Lemma 8.2 shows that the finite-dimensional vector space $E_{\nu}^{-} \oplus \operatorname{ker} A \oplus E_{\nu}^{+}$ is a symplectic subspace of $L^{2}\left(\Omega_{\partial X}^{*}\right)$.

Let $R_{\nu}\left(L_{X}\right) \subset E_{\nu}^{-} \oplus \operatorname{ker} A \oplus E_{\nu}^{+}$be the symplectic reduction of $L_{X}$ with respect to the isotropic subspace $F_{\nu}^{-}$as in Lemma 8.2:

$$
\begin{aligned}
R_{\nu}\left(L_{X}\right) & =\frac{L_{X} \cap\left(F_{\nu}^{-} \oplus E_{\nu}^{-} \oplus \operatorname{ker} A \oplus E_{\nu}^{+}\right)}{L_{X} \cap F_{\nu}^{-}} \\
& =\operatorname{proj}_{E_{\nu}^{-} \oplus \operatorname{ker} A \oplus E_{\nu}^{+}}\left(L_{X} \cap\left(F_{\nu}^{-} \oplus E_{\nu}^{-} \oplus \operatorname{ker} A \oplus E_{\nu}^{+}\right)\right) .
\end{aligned}
$$

Then $R_{\nu}\left(L_{X}\right)$ is a Lagrangian subspace of $E_{\nu}^{-} \oplus \operatorname{ker} A \oplus E_{\nu}^{+}$.

Nicolaescu's theorem [26, Theorem 4.9] says

$$
\lim _{r \rightarrow \infty} L_{X}^{r}=\left(\lim _{r \rightarrow \infty} e^{r A} R_{\nu}\left(L_{X}\right)\right) \oplus F_{\nu}^{+}
$$

(The sign in the exponent $e^{r A}$ differs from [26] because in that paper the collar of $X_{r}$ is parameterized as $\partial X \times[0, r]$.) Thus we need only to identify the limit of $e^{r A} R_{\nu}\left(L_{X}\right)$. To help with the rest of the argument the reader should observe that the dynamics of $e^{r A}$ favor the vectors with a non-zero component in eigenspaces corresponding to positive eigenvalues.

Let $\mu_{1}<\mu_{2}<\cdots<\mu_{q}$ denote the complete list of eigenvalues of $A$ in the range $[-\nu, \nu]$. Thus

$$
E_{\nu}^{-} \oplus \operatorname{ker} A \oplus E_{\nu}^{+}=E_{\mu_{1}} \oplus E_{\mu_{2}} \oplus \cdots \oplus E_{\mu_{q}} .
$$

Given $\ell \in R_{\nu}\left(L_{X}\right)$, we use this decomposition to write

$$
\ell=\left(\ell_{1}, \ell_{2}, \cdots, \ell_{q}\right) \text {. }
$$

Let $\mu(\ell)$ denote the largest $\mu_{i}$ so that $\ell_{i}$ is non-zero (and hence $\ell_{\mu(\ell)+1}=\cdots=\ell_{q}=$ $0)$. Then

$$
\lim _{r \rightarrow \infty} e^{r A}\left(\frac{1}{e^{r \mu(\ell)}} \ell\right)=\left(0,0, \cdots, 0, \ell_{\mu(\ell)}, 0, \cdots, 0\right) .
$$

This shows that

$$
\lim _{r \rightarrow \infty} e^{r A} R_{\nu}\left(L_{X}\right)=L_{\mu_{1}} \oplus L_{\mu_{2}} \oplus \cdots \oplus L_{\mu_{q}} \subset E_{\mu_{1}} \oplus E_{\mu_{2}} \oplus \cdots \oplus E_{\mu_{q}}
$$

where

$$
\begin{aligned}
L_{\mu_{i}} & =\operatorname{proj}_{E_{\mu_{i}}}\left(R_{\nu}\left(L_{X}\right) \cap\left(E_{\mu_{1}} \oplus \cdots \oplus E_{\mu_{i}}\right)\right) \\
& =\operatorname{proj}_{E_{\mu_{i}}}\left(L_{X} \cap\left(F_{\nu}^{-} \oplus E_{\mu_{1}} \oplus \cdots \oplus E_{\mu_{i}}\right)\right) .
\end{aligned}
$$


Write

$$
\begin{aligned}
L^{-} & =\bigoplus_{\mu_{j}<0} L_{\mu_{j}} \subset E_{\nu}^{-}, \\
L^{0} & =\bigoplus_{\mu_{j}=0} L_{\mu_{j}} \subset \operatorname{ker} A,
\end{aligned}
$$

and

$$
L^{+}=\underset{\mu_{j}>0}{\bigoplus} L_{\mu_{j}} \subset E_{\nu}^{-},
$$

so that

$$
\lim _{r \rightarrow \infty} e^{r A} R_{\nu}\left(L_{X}\right)=L^{-} \oplus L^{0} \oplus L^{+} \subset E_{\nu}^{-} \oplus \operatorname{ker} A \oplus E_{\nu}^{+} .
$$

Set

$$
W_{\alpha}:=L^{-}
$$

LEMMA 8.6.

1. $L_{0}=V_{\alpha}$.

2. The spaces

(a) $W_{\alpha}=L^{-}$,

(b) the image of $H^{\text {even }}\left(X, \partial X ; \mathbb{C}_{\alpha}^{n}\right) \rightarrow H^{\text {even }}\left(X ; \mathbb{C}_{\alpha}^{n}\right)$,

(c) $L_{X} \cap F_{0}^{-}$, and

(d) the $L^{2}$ solutions of $D x=0$ on $X_{\infty}$ are all isomorphic.

3. $L^{-} \subset d\left(E_{\nu}^{+}\right)$.

Assuming these three facts, the rest of the proof of Theorem 8.5 is completed as follows.

Note that $W_{\alpha} \subset d\left(E_{\nu}^{+}\right) \subset d\left(E_{\nu}^{+}\right) \oplus d^{*}\left(E_{\nu}^{+}\right)=E_{\nu}^{-}$. We define $W_{\alpha}^{\perp}$ to be the orthogonal complement of $W_{\alpha}$ in $d\left(E_{\nu}^{+}\right)$. Since

$$
W_{\alpha} \oplus L^{+}=L^{-} \oplus L^{+} \subset E_{\nu}^{-} \oplus E_{\nu}^{+}=\left(d\left(E_{\nu}^{+}\right) \oplus d^{*}\left(E_{\nu}^{+}\right)\right) \oplus\left(d\left(E_{\nu}^{-}\right) \oplus d^{*}\left(E_{\nu}^{-}\right)\right)
$$

is a Lagrangian subspace (obtained by modding out $L^{0}$ and $\operatorname{ker} A$ ), it follows from Lemma 8.1 that

$$
L^{+}=d\left(E_{\nu}^{-}\right) \oplus \gamma\left(W_{\alpha}^{\perp}\right) \subset d\left(E_{\nu}^{-}\right) \oplus d^{*}\left(E_{\nu}^{-}\right),
$$

completing the proof of Theorem 8.5.

ProOF OF LEMma 8.6. The first assertion follows immediately by comparing Equations 8.9 and 8.14 .

For the second assertion, Equation (8.14) shows that if $m \in L^{-}$, there exists a $\mu_{i}<0$ and an

$$
\ell=\left(f, \ell_{\mu_{1}}, \ell_{\mu_{2}} \cdots, \ell_{\mu_{i}}\right) \in\left(F_{\nu}^{-} \oplus E_{\mu_{1}} \oplus E_{\mu_{2}} \oplus \cdots \oplus E_{\mu_{i}}\right) \cap L_{X}
$$

with $m=\ell_{\mu_{i}}$. This sets up an identification of $L^{-}$with $F_{0}^{-} \cap L_{X}$. The unique continuation property identifies this (via the restriction map $r$ ) with the kernel of $D$ with $P_{\geq 0}$ boundary conditions, which, by Proposition 8.3 and Equation 8.7 (with $k=0$ ), is the same as the space of $L^{2}$ harmonic forms in $\Omega_{X_{\infty}}^{\text {even }}$. The space of $L^{2}$ harmonic $p$-forms is 
shown to be isomorphic to the image of $H^{p}\left(X, \partial X ; \mathbb{C}_{\alpha}^{n}\right) \rightarrow H^{p}\left(X ; \mathbb{C}_{\alpha}^{n}\right)$ in $\mathbb{1}$, Proposition 4.9].

The third assertion also follows, since if $\ell=r(\beta)$, then Proposition 8.3 says $d \ell=$ $d(r(\beta))=0$. But

$$
0=d \ell=d f+d \ell_{\mu_{1}}+d \ell_{\mu_{2}}+\cdots+d \ell_{\mu_{i}}
$$

and since $d\left(E_{\mu}\right) \subset E_{-\mu}$,

Hence (since $\left.\mu_{i}<0\right)$

$$
0=d \ell_{\mu_{i}}=d m
$$

$$
m \in \operatorname{ker}\left(d: E_{\mu_{i}} \rightarrow E_{-\mu_{i}}\right)=d\left(E_{-\mu_{i}}\right) \subset d\left(E_{\nu}^{+}\right),
$$

completing the proof of Lemma 8.6

REMARK 8.7. Notice that $W_{\alpha}^{\perp}$ denotes the orthogonal complement to $W_{\alpha}$ in the finite-dimensional space $d\left(E_{\nu}^{+}\right)$, not in $L^{2}\left(\Omega_{\partial X}^{*}\right)$.

We adopt the following notation in the rest of this section to deal with boundary conditions. Given a manifold with boundary $X$, the odd signature operator $D=D_{B}$ coupled to a flat connection $B$ on $X$ as above, and a Lagrangian subspace $L \in \mathscr{L}_{\text {Fred }}$, then let $\eta(D, X ; L)$ denote the $\eta$-invariant of the Dirac operator $D$ with boundary conditions given by the orthogonal projection to $L$. Thus,

$$
\eta(D, X ; L):=\eta\left(D_{\operatorname{proj}_{L}}, X\right)
$$

in the previous notation. The same notation applies to the reduced $\eta$-invariant $\widetilde{\eta}$.

In a similar manner, given appropriate Lagrangian subspaces $L, M, N$ of a Hermitian symplectic Hilbert space we will use $\tau_{\mu}(L, M, N)$ to denote the triple index of the corresponding projections $\tau_{\mu}\left(\operatorname{proj}_{L}, \operatorname{proj}_{M}, \operatorname{proj}_{N}\right)$ (cf. Section 6.2).

Suppose that $B$ and $B^{\prime}$ are flat connections on $X$ in temporal gauge near $\partial X$ such that the holonomy representations $\alpha, \alpha^{\prime}: \pi_{1} X \rightarrow U(n)$ of $B, B^{\prime}$ are conjugate. Then there exists a gauge transformation $g$ so that on a collar $[0, \epsilon) \times \partial X, g=\pi^{*}(h)$ for a gauge transformation $h$ on $\partial X$ satisfying $B^{\prime}=g \cdot B$. Hence the restrictions $b, b^{\prime}$ of $B, B^{\prime}$ to the boundary satisfy $b^{\prime}=h \cdot b$. We have

$$
D_{B^{\prime}}=D_{g B}=g D_{B} g^{-1}
$$

and

$$
A_{b^{\prime}}=A_{h b}=h A_{b} h^{-1} .
$$

In particular, $h$ takes the positive (resp. negative) eigenspan of $A_{b}$ to the positive (resp. negative) eigenspan of $A_{b^{\prime}}$, and gives an isomorphism $\operatorname{ker} A_{b} \rightarrow \operatorname{ker} A_{b^{\prime}}$ which coincides via the Hodge and DeRham theorems with the isomorphism $H^{*}\left(\partial X ; \mathbb{C}_{\alpha}^{n}\right) \rightarrow$ $H^{*}\left(\partial X ; \mathbb{C}_{\alpha^{\prime}}^{n}\right)$ induced by conjugating the holonomies $\alpha, \alpha^{\prime}$. Thus if $K \subset \operatorname{ker} A_{b}$ is a Lagrangian subspace, the $\lambda$-eigenspace of $D_{B}$ with $F_{0}^{+}(b) \oplus K$ boundary conditions is sent by $g$ to the $\lambda$-eigenspace of $D_{B^{\prime}}$ with $F_{0}^{+}\left(b^{\prime}\right) \oplus h(K)$ boundary conditions.

Since any representation $\alpha: \pi_{1} X \rightarrow U(n)$ is the holonomy representation of a flat connection $B$, we conclude that given a representation $\alpha$ and a Lagrangian subspace $K \subset H^{*}\left(\partial X ; \mathbb{C}_{\alpha}^{n}\right)$ (recall that the symplectic structure $\omega$ on $H^{*}\left(\partial X ; \mathbb{C}_{\alpha}^{n}\right)$ is defined 
by the cup product), the quantity $\eta\left(D, X ; F_{0}^{+} \oplus K\right)$ is unambiguously defined, i.e. it is independent of the choice of flat connection $B$ in temporal gauge with holonomy conjugate to $\alpha$, and the Lagrangian in ker $A_{b}$ corresponding to $K$ via the Hodge and DeRham theorems is well defined. Of course $\eta\left(D, X ; F_{0}^{+} \oplus K\right)$ may depend on the choice of Riemannian metric on $X$.

We can now turn to the splitting problem for the $\eta$-invariant of the odd signature operator. As in earlier sections suppose that $M=M^{+} \cup M^{-}$is a closed manifold decomposed into 2 submanifolds along a separating hypersurface $N$. Assume that $N$ has a neighborhood isometric to $N \times[-1,1]$. Suppose that $B$ is a flat connection on $M$ in temporal gauge on $N \times[-1,1]$.

As we have seen, because the outward normal for $M^{+}$is the inward normal for $M^{-}$, the operators $\gamma$ and $A$ for $M^{-}$are related to those for $M^{+}$by a change in signs. This has the following consequences. First, whereas the conclusion of Theorem 8.5 identifies the limit of the Cauchy data spaces $L_{M^{+}}^{r}$ of $D$ acting on $M_{r}^{+}, \lim _{r \rightarrow \infty} L_{M^{+}}^{r}$ as

$$
F_{\nu}^{+} \oplus\left(W_{+, \alpha} \oplus \gamma\left(W_{+, \alpha}^{\perp}\right)\right) \oplus d\left(E_{\nu}^{-}\right) \oplus V_{+, \alpha}
$$

(in the decomposition (8.11) for $W_{+, \alpha} \subset d\left(E_{\nu}^{+}\right) \subset F_{0}^{-}$a space isomorphic to the image

$$
\operatorname{im}\left(H^{\text {even }}\left(M^{+}, \partial M^{+} ; \mathbb{C}_{\alpha}^{n}\right) \rightarrow H^{\text {even }}\left(M^{+} ; \mathbb{C}_{\alpha}^{n}\right)\right)
$$

and $V_{+, \alpha} \subset \operatorname{ker} A$ a space isomorphic to

$$
\operatorname{im}\left(H^{\text {even }}\left(M^{+} ; \mathbb{C}_{\alpha}^{n}\right) \rightarrow H^{\text {even }}\left(N ; \mathbb{C}_{\alpha}^{n}\right)\right) .
$$

For $M^{-}$the conclusion is that $\lim _{r \rightarrow \infty} L_{M^{-}}^{r}$ is

$$
F_{\nu}^{-} \oplus d\left(E_{\nu}^{+}\right) \oplus\left(\gamma\left(W_{-, \alpha}^{\perp}\right) \oplus W_{-, \alpha}\right) \oplus V_{-, \alpha},
$$

where $W_{-, \alpha} \subset d\left(E_{\nu}^{-}\right) \subset F_{0}^{+}$is isomorphic to the image

$$
\operatorname{im}\left(H^{\text {even }}\left(M^{-}, \partial M^{-} ; \mathbb{C}_{\alpha}^{n}\right) \rightarrow H^{\text {even }}\left(M^{-} ; \mathbb{C}_{\alpha}^{n}\right)\right)
$$

and $V_{-, \alpha} \subset$ ker $A$ is a space isomorphic to

$$
\operatorname{im}\left(H^{\text {even }}\left(M^{-} ; \mathbb{C}_{\alpha}^{n}\right) \rightarrow H^{\text {even }}\left(N ; \mathbb{C}_{\alpha}^{n}\right)\right)
$$

(We assume that $\nu$ has been chosen greater than or equal to the non-resonance level for $D$ acting on both $M^{+}$and $M^{-}$.)

Theorem 7.7 calculates the $\eta$-invariant of $D$ acting on $M$ in terms of the $\eta$-invariants of $D$ on $M^{+}$and $M^{-}$. Take $P$ to be the Atiyah-Patodi-Singer boundary projection $P=P^{+}(V)$ for some Lagrangian subspace $V \subset \operatorname{ker} A$. Then Theorem 7.7 says

$$
\begin{aligned}
\widetilde{\eta}(D, M)= & \widetilde{\eta}\left(D, M^{+} ; V \oplus F_{0}^{+}\right)+\widetilde{\eta}\left(D, M^{-} ; F_{0}^{-} \oplus \gamma(V)\right) \\
& -\tau_{\mu}\left(I-P_{M^{-}}, P^{+}(V), P_{M^{+}}\right) .
\end{aligned}
$$

(Recall that $P_{M^{ \pm}}$denotes the Calderón projectors onto the Cauchy data spaces $L_{M^{ \pm}}$.) 
THEOREM 8.8. Let $D$ denote the odd signature operator coupled to a flat connection. For any Lagrangian subspace $V \subset \operatorname{ker} A$,

$$
\widetilde{\eta}(D, M)=\widetilde{\eta}\left(D, M^{+} ; V \oplus F_{0}^{+}\right)+\widetilde{\eta}\left(D, M^{-} ; F_{0}^{-} \oplus \gamma(V)\right)-\tau_{\mu}\left(\gamma\left(V_{-, \alpha}\right), V, V_{+, \alpha}\right),
$$

where $\tau_{\mu}\left(\gamma\left(V_{-, \alpha}\right), V, V_{+, \alpha}\right)$ refers to the triple index in the finite-dimensional space ker $A \cong H^{*}\left(N ; \mathbb{C}_{\alpha}^{n}\right)$.

The triple index $\tau_{\mu}\left(\gamma\left(V_{-, \alpha}\right), V, V_{+, \alpha}\right)$ vanishes if $V=V_{+, \alpha}$ or $V=\gamma\left(V_{-, \alpha}\right)$ and so

$$
\begin{aligned}
\widetilde{\eta}(D, M) & =\widetilde{\eta}\left(D, M^{+} ; V_{+, \alpha} \oplus F_{0}^{+}\right)+\widetilde{\eta}\left(D, M^{-} ; F_{0}^{-} \oplus \gamma\left(V_{+, \alpha}\right)\right) \\
& =\widetilde{\eta}\left(D, M^{+} ; \gamma\left(V_{-, \alpha}\right) \oplus F_{0}^{+}\right)+\widetilde{\eta}\left(D, M^{-} ; F_{0}^{-} \oplus V_{-, \alpha}\right) .
\end{aligned}
$$

In particular, if $H^{*}\left(N, \mathbb{C}_{\alpha}^{n}\right)=0$, then

$$
\widetilde{\eta}(D, M)=\widetilde{\eta}\left(D, M^{+} ; F_{0}^{+}\right)+\widetilde{\eta}\left(D, M^{-} ; F_{0}^{-}\right) .
$$

The main advantage that Theorem 8.8 has over Theorem 7.7 is that the Calderón projectors have been replaced by the Atiyah-Patodi-Singer projections.

We postpone the proof of Theorem 8.8 until after two lemmas are in place. The basic idea is to apply Lemma 6.10 to the paths obtained by stretching the Cauchy data spaces to their adiabatic limits.

LEMMA 8.9. $W_{+, \alpha} \oplus W_{-, \alpha} \oplus\left(V_{+, \alpha} \cap V_{-, \alpha}\right)$ is isomorphic to $H^{\text {even }}\left(M ; \mathbb{C}_{\alpha}^{n}\right)$.

Proof. For each integer $k$ let (twisted coefficients in $\mathbb{C}_{\alpha}^{n}$ are to be understood for all cohomology groups)

$$
W_{ \pm}^{k}=\operatorname{im}\left(H^{k}\left(M^{ \pm}, \partial M^{ \pm}\right) \rightarrow H^{k}\left(M^{ \pm}\right)\right)=\operatorname{ker}\left(i_{ \pm}^{*}: H^{k}\left(M^{ \pm}\right) \rightarrow H^{k}(N)\right)
$$

and let

Consider the map

$$
V_{ \pm}^{k}=\operatorname{im}\left(i_{ \pm}^{*}: H^{k}\left(M^{ \pm}\right) \rightarrow H^{k}(N)\right)
$$

$$
\Psi^{k}: H^{k}\left(M^{+}\right) \oplus H^{k}\left(M^{-}\right) \rightarrow H^{k}(N), \quad \Psi^{k}\left(m_{+}, m_{-}\right)=i_{+}^{*}\left(m_{+}\right)-i_{-}^{*}\left(m_{-}\right)
$$

in the Mayer-Vietoris sequence for $M=M^{+} \cup_{N} M^{-}$. Then there is a short exact sequence

$$
0 \rightarrow W_{+}^{k} \oplus W_{-}^{k} \rightarrow \operatorname{ker} \Psi^{k} \stackrel{\beta}{\longrightarrow} V_{+}^{k} \cap V_{-}^{k} \rightarrow 0,
$$

where $\beta\left(m_{+}, m_{-}\right)=i_{1}^{*}\left(m_{+}\right)=i_{2}^{*}\left(m_{-}\right)$. Moreover, the Mayer-Vietoris sequence gives a short exact sequence

$$
0 \rightarrow \operatorname{coker} \Psi^{k-1} \rightarrow H^{k}(M) \rightarrow \operatorname{ker} \Psi^{k} \rightarrow 0 .
$$

Thus

$$
\operatorname{dim} H^{k}(M)=\operatorname{dim} \text { coker } \Psi^{k-1}+\operatorname{dim}\left(V_{+}^{k} \cap V_{-}^{k}\right)+\operatorname{dim} W_{+}^{k}+\operatorname{dim} W_{-}^{k} .
$$

Also

$$
\begin{aligned}
& \operatorname{dim} \text { coker } \Psi^{k-1}=\operatorname{dim} H^{k-1}(N) /\left(V_{+}^{k-1}+V_{-}^{k-1}\right) \\
& \quad=\operatorname{dim} H^{k-1}(N)-\operatorname{dim} V_{+}^{k-1}-\operatorname{dim} V_{-}^{k-1}+\operatorname{dim}\left(V_{+}^{k-1} \cap V_{-}^{k-1}\right) .
\end{aligned}
$$


Combining (8.21) and (8.22) and summing up over $k$ even one obtains

$$
\begin{aligned}
\sum \operatorname{dim} H^{2 k}(M)= & \sum \operatorname{dim}\left(V_{+}^{k} \cap V_{-}^{k}\right)+\sum \operatorname{dim} W_{+}^{2 k}+\sum \operatorname{dim} W_{-}^{2 k} \\
& +\sum \operatorname{dim} H^{2 k-1}(N)-\sum \operatorname{dim} V_{+}^{2 k-1}-\sum \operatorname{dim} V_{-}^{2 k-1} .
\end{aligned}
$$

The symplectic space $H^{*}(N)$ decomposes as a symplectic sum $H^{\text {even }}(N) \oplus H^{\text {odd }}(N)$ (one way to see this is to notice that $\hat{*}$ and hence $\gamma$ preserves the parity of a harmonic form since $N$ is $2 n$-dimensional). The Lagrangian subspace $V_{+}^{*}=\sum V_{+}^{k}$ decomposes accordingly into a sum of Lagrangian subspaces $\oplus V_{+}^{2 k} \oplus V_{+}^{2 k-1}$. Hence $\operatorname{dim}\left(\oplus V_{+}^{2 k-1}\right)=$ $\frac{1}{2} \operatorname{dim} H^{\text {odd }}(N)$. Similarly $\operatorname{dim}\left(\oplus V_{-}^{2 k-1}\right)=\frac{1}{2} \operatorname{dim} H^{\text {odd }}(N)$. Thus the last three terms in (8.23) cancel. Since $W_{ \pm, \alpha}=\oplus W_{ \pm}^{2 k}$ and $V_{ \pm, \alpha}=\oplus V_{ \pm}^{k}$,

$$
\operatorname{dim} H^{\text {even }}(M)=\operatorname{dim}\left(V_{+, \alpha} \cap V_{-, \alpha}\right)+\operatorname{dim} W_{+, \alpha}+\operatorname{dim} W_{-, \alpha},
$$

completing the proof of Lemma 8.9.

Lemma 8.10. Let $V \subset \operatorname{ker} A$ be a Lagrangian subspace. Let $L_{M^{ \pm}}^{r}$ denote the Cauchy data space for $D$ acting on $M_{r}^{ \pm}=([-r, 0] \times N) \cup M^{ \pm}$when $r<\infty$ and let $L_{M^{ \pm}}^{\infty}$ be the adiabatic limit $\lim _{r \rightarrow \infty} L_{M^{ \pm}}^{r}$ which was identified in Theorem 8.5 .

1. The dimension of the intersection $L_{M^{-}}^{r} \cap L_{M^{+}}^{r}$ is independent of $r \in[0, \infty]$.

2. The dimension of the intersection $L_{M^{-}}^{r} \cap\left(F_{0}^{+} \oplus V\right)$ is independent of $r \in[0, \infty]$.

3. The dimension of the intersection $\left(F_{0}^{-} \oplus \gamma(V)\right) \cap L_{M^{+}}^{r}$ is independent of $r \in[0, \infty]$.

4. $\tau_{\mu}\left(\gamma\left(L_{M^{-}}^{\infty}\right), F_{0}^{+} \oplus V, L_{M^{+}}^{\infty}\right)=\tau_{\mu}\left(\gamma\left(V_{-, \alpha}\right), V, V_{+, \alpha}\right)$.

PROOF. 1. For $r<\infty$, the intersection $L_{M^{-}}^{r} \cap L_{M^{+}}^{r}$ is isomorphic to the kernel of $D_{B}$ acting on the closed manifold $M_{r}=M_{r}^{+} \cup M_{r}^{-}$obtained by stretching the collar of $N$. But this kernel is a homotopy invariant, isomorphic to $H^{\text {even }}\left(M ; \mathbb{C}_{\alpha}^{n}\right)$, and in particular its dimension is independent of $r$.

To compute $L_{M^{-}}^{\infty} \cap L_{M^{+}}^{\infty}$, we use Theorem 8.5, or, more conveniently, its consequences (8.16) and (8.17). These show that $L_{M^{-}}^{\infty} \cap L_{M^{+}}^{\infty}=W_{+, \alpha} \oplus W_{-, \alpha} \oplus\left(V_{+, \alpha} \cap V_{-, \alpha}\right)$, which by Lemma 8.9 is also isomorphic to $H^{\text {even }}\left(M ; \mathbb{C}_{\alpha}^{n}\right)$. Notice that the full conclusion of Theorem 8.5 is used here.

2. and 3. are proven by the same argument. We prove 3. From the definition of $V_{+, \alpha}(8.9)$ there is an exact sequence

$$
0 \rightarrow L_{M^{+}} \cap F_{0}^{-} \rightarrow L_{M^{+}} \cap\left(F_{0}^{-}+\operatorname{ker} A\right) \rightarrow V_{+, \alpha} \rightarrow 0 .
$$

It follows easily that for any subspace $V \subset \operatorname{ker} A$ there is an exact sequence

$$
0 \rightarrow L_{M^{+}} \cap F_{0}^{-} \rightarrow L_{M^{+}} \cap\left(F_{0}^{-}+\gamma(V)\right) \rightarrow V_{+, \alpha} \cap \gamma(V) \rightarrow 0 .
$$

Lemma 8.6 identifies $L_{M^{+}} \cap F_{0}^{-}$with the image

$$
H^{\text {even }}\left(M^{+}, \partial M^{+} ; \mathbb{C}_{\alpha}^{n}\right) \rightarrow H^{\text {even }}\left(M^{+} ; \mathbb{C}_{\alpha}^{n}\right),
$$

and with $W_{+, \alpha}$. Thus the dimension of $L_{M^{+}} \cap F_{0}^{-}$is independent of the length of the collar of $M^{+}$. Corollary 8.4 identifies $V_{+, \alpha}$ with the image of $H^{*}\left(M^{+} ; \mathbb{C}_{\alpha}^{n}\right) \rightarrow$ $H^{*}\left(\partial M^{+} ; \mathbb{C}_{\alpha}^{n}\right)$, hence its intersection with $\gamma(V)$ is independent of the length of the collar as well. Thus the middle term in the exact sequence (8.24) is isomorphic to 
$W_{+, \alpha} \oplus\left(V_{+, \alpha} \cap \gamma(V)\right)$ and in particular its dimension is independent of the length of the collar; this shows that $\left(F_{0}^{-} \oplus \gamma(V)\right) \cap L_{M^{+}}^{r}$ is independent of $r$ for $r<\infty$.

Now consider the case when $r=\infty$. In the decomposition (8.11) of $L^{2}\left(\Omega_{N}^{*}\right)$,

$$
F_{0}^{-} \oplus \gamma(V)=F_{\nu}^{-} \oplus d\left(E_{\nu}^{+}\right) \oplus d^{*}\left(E_{\nu}^{+}\right) \oplus \gamma(V)
$$

and so using (8.16) shows that $\left(F_{0}^{-} \oplus \gamma(V)\right) \cap L_{M^{+}}^{\infty}$ equals

$$
\begin{gathered}
\left(F_{\nu}^{-} \oplus d\left(E_{\nu}^{+}\right) \oplus d^{*}\left(E_{\nu}^{+}\right) \oplus \gamma(V)\right) \cap\left(F_{\nu}^{+} \oplus\left(W_{+, \alpha} \oplus \gamma\left(W_{+, \alpha}^{\perp}\right)\right) \oplus d\left(E_{\nu}^{-}\right) \oplus V_{+, \alpha}\right) \\
=W_{+, \alpha} \oplus\left(\gamma(V) \cap V_{+, \alpha}\right) .
\end{gathered}
$$

Therefore, $\left(F_{0}^{-} \oplus \gamma(V)\right) \cap L_{M^{+}}^{\infty}$ equals to $W_{+, \alpha} \oplus\left(V_{+, \alpha} \cap \gamma(V)\right)$.

4. It follows immediately from the definition that the triple index is additive in the following sense: let $H, H^{\prime}$ be Hermitian symplectic Hilbert spaces and $P, Q, R$ (resp. $\quad P^{\prime}, Q^{\prime}, R^{\prime}$ ) be projections in $\operatorname{Gr}(H)$ (resp. $\operatorname{Gr}\left(H^{\prime}\right)$ ) such that the triple indices $\tau_{\mu}(P, Q, R), \tau_{\mu}\left(P^{\prime}, Q^{\prime}, R^{\prime}\right)$ are well-defined. Then the triple index of $\left(P \oplus P^{\prime}, Q \oplus Q^{\prime}, R \oplus R^{\prime}\right)$ is well-defined in the Hermitian symplectic Hilbert space $H \oplus H^{\prime}$ and we have

$$
\tau_{\mu}\left(P \oplus P^{\prime}, Q \oplus Q^{\prime}, R \oplus R^{\prime}\right)=\tau_{\mu}(P, Q, R)+\tau_{\mu}\left(P^{\prime}, Q^{\prime}, R^{\prime}\right) .
$$

In the decomposition 8.11) we have

$$
\begin{aligned}
\gamma\left(L_{M^{-}}^{\infty}\right) & =\gamma\left(F_{\nu}^{-} \oplus d\left(E_{\nu}^{+}\right) \oplus\left(\gamma\left(W_{-, \alpha}^{\perp}\right) \oplus W_{-, \alpha}\right) \oplus V_{-, \alpha}\right) \\
& =F_{\nu}^{+} \oplus d^{*}\left(E_{\nu}^{-}\right) \oplus\left(W_{-, \alpha}^{\perp} \oplus \gamma\left(W_{-, \alpha}\right)\right) \oplus \gamma\left(V_{-, \alpha}\right), \\
F_{0}^{+} \oplus V & =F_{\nu}^{+} \oplus d^{*}\left(E_{\nu}^{-}\right) \oplus d\left(E_{\nu}^{-}\right) \oplus V, \text { and } \\
L_{M^{+}}^{\infty} & =F_{\nu}^{+} \oplus\left(W_{+, \alpha} \oplus \gamma\left(W_{+, \alpha}^{\perp}\right) \oplus d\left(E_{\nu}^{-}\right) \oplus V_{+, \alpha} .\right.
\end{aligned}
$$

Using the additivity of $\tau_{\mu}$ we see that $\tau_{\mu}\left(\gamma\left(L_{M^{-}}^{\infty}\right), F_{0}^{+} \oplus V, L_{M^{+}}^{\infty}\right)$ equals

$$
\begin{aligned}
& \tau_{\mu}\left(F_{\nu}^{+}, F_{\nu}^{+}, F_{\nu}^{+}\right)_{F_{\nu}^{-} \oplus F_{\nu}^{+}}+\tau_{\mu}\left(d^{*}\left(E_{\nu}^{-}\right), d^{*}\left(E_{\nu}^{-}\right), W_{+, \alpha} \oplus \gamma\left(W_{+, \alpha}^{\perp}\right)\right)_{d\left(E_{\nu}^{+}\right) \oplus d^{*}\left(E_{\nu}^{-}\right)} \\
& \quad+\tau_{\mu}\left(\gamma\left(W_{-, \alpha}^{\perp}\right) \oplus W_{-, \alpha}, d\left(E_{\nu}^{-}\right), d\left(E_{\nu}^{-}\right)\right)_{d^{*}\left(E_{\nu}^{+}\right) \oplus d\left(E_{\nu}^{-}\right)}+\tau_{\mu}\left(\gamma\left(V_{-, \alpha}\right), V, V_{+, \alpha}\right)_{\operatorname{ker} A}
\end{aligned}
$$

which equals $\tau_{\mu}\left(\gamma\left(V_{-, \alpha}\right), V, V_{+, \alpha}\right)_{\text {ker } A}$ by Proposition 6.11.

Proof of Theorem 8.8. Combining Lemma 8.10 and Lemma 6.10 to the paths of Cauchy data spaces obtained by stretching the collars of $M^{ \pm}$to their adiabatic limit, we see that (switching from projection to Lagrangian notation)

$$
\tau_{\mu}\left(I-P_{M^{-}}, P^{+}(V), P_{M_{+}}\right)=\tau_{\mu}\left(\gamma\left(L_{M^{-}}^{\infty}\right), F_{0}^{+} \oplus V, L_{M^{+}}^{\infty}\right)=\tau_{\mu}\left(\gamma\left(V_{-, \alpha}\right), V, V_{+, \alpha}\right) .
$$

It then follows from Theorem 7.7 (and in particular (8.18)) that

$$
\widetilde{\eta}(D, M)=\widetilde{\eta}\left(D_{B}, M^{+} ; V \oplus F_{0}^{+}\right)+\widetilde{\eta}\left(D, M^{-} ; F_{0}^{-} \oplus \gamma(V)\right)-\tau_{\mu}\left(\gamma\left(V_{-, \alpha}\right), V, V_{+, \alpha}\right) .
$$

Proposition 6.11 shows that $\tau_{\mu}\left(\gamma\left(V_{-, \alpha}\right), V, V_{+, \alpha}\right)=0$ if $V=\gamma\left(V_{-, \alpha}\right)$ or $V_{+, \alpha}$. 
REMARK 8.11. The Riemannian metric on the separating hypersurface $N$ enters into the formula of Theorem 8.8 via the map $\gamma: \operatorname{ker} A \rightarrow \operatorname{ker} A$, since $\gamma$ equals the Hodge $-*$ operator up to a power of $i$. It follows that the correction term $\tau_{\mu}\left(\gamma\left(V_{-, \alpha}\right), V, V_{+, \alpha}\right)$ is not a homotopy invariant. In fact, suppose that varying the Riemannian metric moves the space $\gamma\left(V_{-, \alpha}\right)$ slightly (we use the Hodge and DeRham theorems to identify this as a subspace of the fixed space $\left.H^{*}\left(N ; \mathbb{C}_{\alpha}^{n}\right)\right)$. Then by choosing $V \subset H^{*}\left(N ; \mathbb{C}_{\alpha}^{n}\right)$ carefully so that $\gamma\left(V_{-, \alpha}\right)$ passes through $V$ as the metric is varied one can change $\tau_{\mu}\left(\gamma\left(V_{-, \alpha}\right), V, V_{+, \alpha}\right)$.

To complete our analysis of the odd signature operator, we derive a formula which calculates $\widetilde{\eta}(D, M)$ in terms of intrinsic invariants of the pieces and a "correction term" which only depends on finite-dimensional data, namely the subspaces $V_{ \pm, \alpha} \subset \operatorname{ker} A$.

First we define the analogue of the map $\Phi: \operatorname{Gr}(A) \rightarrow \mathscr{U}\left(\mathscr{E}_{i}, \mathscr{E}_{-i}\right)$ of Equation (2.7) in the finite-dimensional space ker $A$. We use the Lagrangian notation, so that to any Lagrangian subspace $K \subset \operatorname{ker} A$ we assign the unitary map

$$
\phi(K):\left(\mathscr{E}_{i} \cap \operatorname{ker} A\right) \rightarrow\left(\mathscr{E}_{-i} \cap \operatorname{ker} A\right)
$$

by the formula

$$
K=\left\{x+\phi(K)(x) \mid x \in\left(\mathscr{E}_{i} \cap \operatorname{ker} A\right)\right\}
$$

THEOREM 8.12. For the odd signature operator $D$ coupled to a flat connection $B$ with holonomy $\alpha: \pi_{1} M \rightarrow U(n)$ acting on a split manifold $M=M^{+} \cup_{N} M^{-}$,

$$
\begin{aligned}
\widetilde{\eta}(D, M)= & \widetilde{\eta}\left(D, M^{+} ; V_{+, \alpha} \oplus F_{0}^{+}\right)+\widetilde{\eta}\left(D, M^{-} ; F_{0}^{-} \oplus V_{-, \alpha}\right) \\
& +\operatorname{dim}\left(V_{+, \alpha} \cap V_{-, \alpha}\right)-\frac{1}{2 \pi i} \operatorname{tr} \log \left(-\phi\left(V_{+, \alpha}\right) \phi\left(V_{-, \alpha}\right)^{*}\right) .
\end{aligned}
$$

REMARK 8.13. Corollary 8.4 implies that $\operatorname{dim}\left(V_{+, \alpha} \cap V_{-, \alpha}\right)$ depends only on the homotopy type of the triple $\left(M, M^{+}, M^{-}\right)$and the representation $\alpha: \pi_{1} M \rightarrow U(n)$.

Proof. Theorem 4.4 implies that

$$
\begin{aligned}
\widetilde{\eta}\left(D, M^{+} ; P^{+}\left(\gamma\left(V_{-, \alpha}\right)\right)\right)= & \widetilde{\eta}\left(D, M^{+} ; P_{M^{+}}\right) \\
& +\frac{1}{2 \pi i} \operatorname{tr} \log \left(\Phi\left(P^{+}\left(\gamma\left(V_{-, \alpha}\right)\right)\right) \Phi\left(P_{M^{+}}\right)^{*}\right)
\end{aligned}
$$

and that

$$
\begin{aligned}
\widetilde{\eta}\left(D, M^{+} ; P^{+}\left(V_{+, \alpha}\right)\right)= & \widetilde{\eta}\left(D, M^{+} ; P_{M^{+}}\right) \\
& +\frac{1}{2 \pi i} \operatorname{tr} \log \left(\Phi\left(P^{+}\left(V_{+, \alpha}\right)\right) \Phi\left(P_{M^{+}}\right)^{*}\right) .
\end{aligned}
$$

Subtracting (8.27) from (8.26) yields

$$
\begin{aligned}
& \widetilde{\eta}\left(D, M^{+} ; P^{+}\left(\gamma\left(V_{-, \alpha}\right)\right)\right)=\widetilde{\eta}\left(D, M^{+} ; P^{+}\left(V_{+, \alpha}\right)\right) \\
& +\frac{1}{2 \pi i} \operatorname{tr} \log \left(\Phi\left(P^{+}\left(\gamma\left(V_{-, \alpha}\right)\right)\right) \Phi\left(P_{M^{+}}\right)^{*}\right)-\frac{1}{2 \pi i} \operatorname{tr} \log \left(\Phi\left(P^{+}\left(V_{+, \alpha}\right)\right) \Phi\left(P_{M^{+}}\right)^{*}\right) .
\end{aligned}
$$

The difference

$$
\frac{1}{2 \pi i} \operatorname{tr} \log \left(\Phi\left(P^{+}\left(\gamma\left(V_{-, \alpha}\right)\right)\right) \Phi\left(P_{M^{+}}\right)^{*}\right)-\frac{1}{2 \pi i} \operatorname{tr} \log \left(\Phi\left(P^{+}\left(V_{+, \alpha}\right)\right) \Phi\left(P_{M^{+}}\right)^{*}\right)
$$

is equal to

$$
\tau_{\mu}\left(P^{+}\left(V_{+, \alpha}\right), P^{+}\left(\gamma\left(V_{-, \alpha}\right)\right), P_{M^{+}}\right)-\frac{1}{2 \pi i} \operatorname{tr} \log \left(\Phi\left(P^{+}\left(V_{+, \alpha}\right)\right) \Phi\left(P^{+}\left(\gamma\left(V_{-, \alpha}\right)\right)\right)^{*}\right)
$$


by Lemma 6.9. Moreover, it follows easily from the definitions that

$$
\operatorname{tr} \log \left(\Phi\left(P^{+}\left(V_{+, \alpha}\right)\right) \Phi\left(P^{+}\left(\gamma\left(V_{-, \alpha}\right)\right)\right)^{*}\right)=\operatorname{tr} \log \left(\phi\left(V_{+, \alpha}\right) \phi\left(\gamma\left(V_{-, \alpha}\right)\right)^{*}\right),
$$

and since $\phi(\gamma(V))=-\phi(V)$, that

$$
\operatorname{tr} \log \left(\phi\left(V_{+, \alpha}\right) \phi\left(\gamma\left(V_{-, \alpha}\right)\right)^{*}\right)=\operatorname{tr} \log \left(-\phi\left(V_{+, \alpha}\right) \phi\left(V_{-, \alpha}\right)^{*}\right) .
$$

Hence 8.28 reduces to

$$
\begin{aligned}
& \widetilde{\eta}\left(D, M^{+} ; P^{+}\left(\gamma\left(V_{-, \alpha}\right)\right)\right)=\widetilde{\eta}\left(D, M^{+} ; P^{+}\left(V_{+, \alpha}\right)\right) \\
& \quad+\tau_{\mu}\left(P^{+}\left(V_{+, \alpha}\right), P^{+}\left(\gamma\left(V_{-, \alpha}\right)\right), P_{M^{+}}\right)-\frac{1}{2 \pi i} \operatorname{tr} \log \left(-\phi\left(V_{+, \alpha}\right) \phi\left(V_{-, \alpha}\right)^{*}\right) .
\end{aligned}
$$

We will show that

$$
\tau_{\mu}\left(P^{+}\left(V_{+, \alpha}\right), P^{+}\left(\gamma\left(V_{-, \alpha}\right)\right), P_{M^{+}}\right)=\operatorname{dim}\left(V_{+, \alpha} \cap V_{-, \alpha}\right) .
$$

Assuming (8.30), the proof of Theorem 8.12 is completed by combining (8.29) and Theorem 8.8, taking $V=\gamma\left(V_{-, \alpha}\right)$.

It remains, therefore, to prove (8.30). The proof is similar to the proof of Theorem 8.8. Lemma 8.10 implies that as the collar of $M^{+}$is stretched to its adiabatic limit, the dimension of the intersection of $L_{M^{+}}^{r}$ with $F_{0}^{+} \oplus V_{+, \alpha}$ is independent of $r \in[0, \infty]$, as is the dimension of the intersection of $L_{M^{+}}^{r}$ with $F_{0}^{+} \oplus \gamma\left(V_{-, \alpha}\right)$.

Lemma 6.10 then implies that $\tau_{\mu}\left(P^{+}\left(V_{+, \alpha}\right), P^{+}\left(\gamma\left(V_{-, \alpha}\right)\right), P_{M^{+}}\right)$is equal to $\tau_{\mu}\left(P^{+}\left(V_{+, \alpha}\right), P^{+}\left(\gamma\left(V_{-, \alpha}\right)\right), P_{M^{+}}^{\infty}\right)$. Using additivity of the triple index with respect to the decomposition (8.11), the calculation of $L_{M^{+}}^{\infty}$ (8.16), and Proposition 6.11, we conclude that

$$
\tau_{\mu}\left(P^{+}\left(V_{+, \alpha}\right), P^{+}\left(\gamma\left(V_{-, \alpha}\right)\right), P_{M^{+}}^{\infty}\right)=\tau_{\mu}\left(V_{+, \alpha}, \gamma\left(V_{-, \alpha}\right), V_{+, \alpha}\right) .
$$

Proposition 6.11 then implies that

$$
\tau_{\mu}\left(V_{+, \alpha}, \gamma\left(V_{-, \alpha}\right), V_{+, \alpha}\right)=\operatorname{dim}\left(V_{+, \alpha} \cap V_{-, \alpha}\right) .
$$

It is convenient to introduce the following notation.

Definition 8.14. Let $(H,\langle\rangle,, \gamma)$ be a finite-dimensional Hermitian symplectic space (cf. Def. 2.8). Define a function of pairs of Lagrangian subspaces

$$
m_{H}: \mathscr{L}(H) \times \mathscr{L}(H) \rightarrow \mathbb{R}
$$

by

$$
\begin{aligned}
m_{H}(V, W) & =-\frac{1}{\pi i} \operatorname{tr} \log \left(-\phi(V) \phi(W)^{*}\right)+\operatorname{dim}(V \cap W) \\
& =-\frac{1}{\pi i} \sum_{\substack{\lambda \in \operatorname{spec}\left(-\phi(V) \phi(W)^{*}\right) \\
\lambda \neq-1}} \log \lambda . \\
&
\end{aligned}
$$

Here $\phi(V)$ is the unitary map from the $+i$ eigenspace $E_{i}$ of $\gamma$ to the $-i$ eigenspace $E_{-i}$ of $\gamma$ whose graph is $V$. (If $H=0$ then set $m_{H}(V, W)=0$.) Recall that $V \cap W$ is isomorphic to the -1 -eigenspace of $\phi(\gamma V) \phi(W)^{*}=-\phi(V) \phi(W)^{*}$ (cf. Lemma 2.6). 
The function $m$ has been investigated before, the notation is taken from [9]

Given an even-dimensional Riemannian manifold $(N, g)$ and a representation $\alpha$ : $\pi_{1} N \rightarrow U(n)$, define

$$
m\left(V_{+}, V_{-}, \alpha, g\right)=m_{H^{*}\left(N ; \mathbb{C}_{\alpha}^{n}\right)}\left(V_{+}, V_{-}\right),
$$

where we have used the Hodge Theorem (and hence the metric $g$ on $N$ ) to identify $H^{*}\left(N ; \mathbb{C}_{\alpha}^{n}\right)$ with ker $A$ (so that $\gamma$ and hence $\phi$ make sense).

Thus Theorem 8.12 says that

$$
\begin{aligned}
\widetilde{\eta}(D, M)=\widetilde{\eta}(D, & \left.M^{+} ; V_{+, \alpha} \oplus F_{0}^{+}\right)+\widetilde{\eta}\left(D, M^{-} ; F_{0}^{-} \oplus V_{-, \alpha}\right) \\
& +\frac{1}{2} \operatorname{dim}\left(V_{+, \alpha} \cap V_{-, \alpha}\right)+\frac{1}{2} m\left(V_{+, \alpha}, V_{-, \alpha}, \alpha, g\right) .
\end{aligned}
$$

Using $\eta$-invariants instead of $\tilde{\eta}$-invariants, (8.31) can be put in the more compact form

$$
\eta(D, M)=\eta\left(D, M^{+} ; V_{+, \alpha} \oplus F_{0}^{+}\right)+\eta\left(D, M^{+} ; F_{0}^{-} \oplus V_{-, \alpha}\right)+m\left(V_{+, \alpha}, V_{-, \alpha}, \alpha, g\right)
$$

using Equation (8.24) and Lemma 8.9.

The function $m_{H}(V, W)$ has some useful properties which we list in the following proposition.

Proposition 8.15.

1. $m_{H}(W, V)=-m_{H}(V, W)$.

2. $m_{H_{1} \oplus H_{2}}\left(V_{1} \oplus V_{2}, W_{1} \oplus W_{2}\right)=m_{H_{1}}\left(V_{1}, W_{1}\right)+m_{H_{2}}\left(V_{2}, W_{2}\right)$.

3. If $h_{t}: H \rightarrow H, t \in[0,1]$ is a continuous family of symplectic automorphisms, then $m_{H}\left(h_{t}(V), h_{t}(W)\right)$ is continuous in $t$.

Proof. The first assertion follows immediately from the definition of $m_{H}$. The second assertion is clear. For the third, notice that $\operatorname{dim}\left(h_{t}(V) \cap h_{t}(W)\right)$ is independent of $t$, and that the -1 eigenspace of $-\phi\left(h_{t}(V)\right) \phi\left(h_{t}(W)\right)^{*}$ is isomorphic to $h_{t}(V) \cap h_{t}(W)$. In particular the -1 eigenspace of $-\phi\left(h_{t}(V)\right) \phi\left(h_{t}(W)\right)^{*}$ is constant dimensional, and so $t \mapsto \log \left(-\phi\left(h_{t}(V)\right) \phi\left(h_{t}(W)\right)^{*}\right)$ is continuous. These facts imply that $m_{H}\left(h_{t}(V), h_{t}(W)\right)$ is continuous in $t$.

\subsection{The Atiyah-Patodi-Singer $\rho_{\alpha}$-invariant for manifolds with bound-} ary. We apply the previous results to obtain information about the Atiyah-PatodiSinger $\rho_{\alpha}$-invariant [2]. Consider two flat connections: $B$ with holonomy $\alpha$, odd signature operator $D_{B}$ and tangential operator $A_{b}$, and the trivial connection $\Theta$ on the bundle $\mathbb{C}^{n} \times X \rightarrow X$ with (trivial) holonomy $\tau$, odd signature operator $D_{\Theta}$, and tangential operator $A_{\theta}$.

In expressions like $\eta\left(D, X ; V \oplus F_{0}^{+}\right)$the notation $F_{0}^{+}$is to be understood as the positive eigenspan of the tangential operator $A$ of $D$ and $V$ as a Lagrangian in ker $A$. In particular, in a "mixed" expression like $\widetilde{\eta}\left(D_{B}, X ; V_{\alpha} \oplus F_{0}^{+}\right)-\widetilde{\eta}\left(D_{\Theta}, X ; V_{\tau} \oplus F_{0}^{+}\right)$the reader should understand that the first $F_{0}^{+}$refers to the positive eigenspan of $A_{b}$ and the second the positive eigenspan of $A_{\theta}$. These are in general unrelated since $A_{b}$ acts on the bundle $E \mid \partial X$ and $A_{\theta}$ acts on the trivial bundle. 
LEMma 8.16. Let $X$ be a Riemannian manifold with boundary, whose collar is isometric to $[0, \varepsilon) \times \partial X$. Let $B$ be a flat connection on a compact manifold $X$ in temporal gauge near the boundary with holonomy $\alpha$, and let $\Theta$ denote the trivial connection, with trivial holonomy $\tau: \pi_{1}(X) \rightarrow U(n)$.

Then the difference

$$
\widetilde{\eta}\left(D_{B}, X ; V_{\alpha} \oplus F_{0}^{+}\right)-\widetilde{\eta}\left(D_{\Theta}, X ; V_{\tau} \oplus F_{0}^{+}\right)
$$

depends only on the diffeomorphism type of $X$, the conjugacy class of the holonomy representation of $B$ and the restriction of the Riemannian metric to $\partial X$.

Proof. We explained above why the $\eta$-invariant depends on the flat connection $B$ only through the conjugacy class of its holonomy representation.

By taking the double of $X$ we obtain a closed Riemannian manifold $M=X \cup-X=$ $M^{+} \cup M^{-}$over which the connections $B$ and $\Theta$ extend flatly.

Letting $D_{B}$ denote the extension to $M$, we know from [2] that the difference

$$
\widetilde{\eta}\left(D_{B}, M\right)-\widetilde{\eta}\left(D_{\Theta}, M\right)
$$

is independent of the metric on $M$ and depends only on the conjugacy class of the holonomy representation of $B$ (see the paragraph following this proof).

Theorem 8.8 shows that

$$
\begin{aligned}
\widetilde{\eta}\left(D_{B}, M\right)-\widetilde{\eta}\left(D_{\Theta}, M\right)= & \widetilde{\eta}\left(D_{B}, M^{+} ; V_{\alpha} \oplus F_{0}^{+}\right)+\widetilde{\eta}\left(D_{B}, M^{-} ; F_{0}^{-} \oplus \gamma\left(V_{\alpha}\right)\right) \\
& -\widetilde{\eta}\left(D_{\Theta}, M^{+} ; V_{\tau} \oplus F_{0}^{+}\right)-\widetilde{\eta}\left(D_{\Theta}, M^{-} ; F_{0}^{-} \oplus \gamma\left(V_{\tau}\right)\right) .
\end{aligned}
$$

Notice that by Corollary 8.4 the subspaces $V_{\alpha}$ and $V_{\tau}$ are independent of the Riemannian metric on $M^{+}$. Hence solving for $\widetilde{\eta}\left(D_{B}, M^{+} ; V_{\alpha} \oplus F_{0}^{+}\right)-\widetilde{\eta}\left(D_{\Theta}, M^{+} ; V_{\tau} \oplus F_{0}^{+}\right)$in (8.35) yields an expression which is unchanged when the Riemannian metric is altered on the interior of $X=M^{+}$.

We can now extend the definition of the Atiyah-Patodi-Singer $\rho_{\alpha}$-invariant to manifolds with boundary. Recall that the $\rho_{\alpha}$-invariant is defined in [2] for a closed manifold $M$ and a representation $\alpha: \pi_{1}(M) \rightarrow U(n)$ by

$$
\rho(M, \alpha)=\eta\left(D_{B}\right)-\eta\left(D_{\Theta}\right)
$$

where $B$ is a flat connection on $M$ with holonomy $\alpha$ and $\Theta$ denotes a trivial $U(n)$ connection. It is a diffeomorphism invariant of the pair $(M,[\alpha])$ where $[\alpha]$ denotes the conjugacy class of $\alpha$. In terms of reduced $\eta$-invariants $\rho(M, \alpha)$ can be written:

$$
\rho(M, \alpha)=2\left(\widetilde{\eta}\left(D_{B}\right)-\widetilde{\eta}\left(D_{\Theta}\right)\right)-\operatorname{dim} \operatorname{ker} D_{B}+\operatorname{dim} \operatorname{ker} D_{\Theta} .
$$

Since ker $D_{B}$ is isomorphic to $H^{\text {even }}\left(M ; \mathbb{C}_{\alpha}^{n}\right)$ this is the same as

$$
\rho(M, \alpha)=2\left(\widetilde{\eta}\left(D_{B}, M\right)-\widetilde{\eta}\left(D_{\Theta}, M\right)\right)-\operatorname{dim} H^{\text {even }}\left(M ; \mathbb{C}_{\alpha}^{n}\right)+\operatorname{dim} H^{\text {even }}\left(M ; \mathbb{C}^{n}\right) .
$$

Definition 8.17. Given a triple $(X, \alpha, g)$, where

1. $X$ is a compact odd-dimensional manifold with boundary,

2. $\alpha: \pi_{1}(X) \rightarrow U(n)$ is a representation, and

3. $g$ is a Riemannian metric on $\partial X$, 
choose a Riemannian metric on $X$ isometric to $[0, \epsilon) \times \partial X$ on a collar of $\partial X$ and a flat connection $B$ with holonomy $\alpha$ in temporal gauge near the boundary. Then define

$$
\rho(X, \alpha, g):=\eta\left(D_{B}, X, F_{0}^{+} \oplus V_{\alpha}\right)-\eta\left(D_{\Theta}, X, F_{0}^{+} \oplus V_{\tau}\right) .
$$

Reversing the orientation of $X$ changes the sign of $\rho(X, \alpha, g)$, since the $\eta$-invariant changes sign when the orientation is reversed.

In terms of reduced $\eta$ invariants $\rho(X, \alpha, g)$ can be expressed as:

$$
\rho(X, \alpha, g)=2\left(\widetilde{\eta}\left(D_{B}, X, F_{0}^{+} \oplus V_{\alpha}\right)-\widetilde{\eta}\left(D_{\Theta}, X, F_{0}^{+} \oplus V_{\tau}\right)\right)-\operatorname{dim} W_{\alpha}+\operatorname{dim} W_{\tau},
$$

where

$$
W_{\alpha} \cong \operatorname{im}\left(H^{\text {even }}\left(X, \partial X ; \mathbb{C}_{\alpha}^{n}\right) \rightarrow H^{\text {even }}\left(X ; \mathbb{C}_{\alpha}^{n}\right)\right)
$$

and

$$
W_{\tau} \cong \operatorname{im}\left(H^{\text {even }}\left(X, \partial X ; \mathbb{C}^{n}\right) \rightarrow H^{\text {even }}\left(X ; \mathbb{C}^{n}\right)\right)
$$

This is because the kernel of $D_{B}$ acting on $X$ with boundary conditions given by the Atiyah-Patodi-Singer projection $P^{+}\left(V_{\alpha}\right)$ is isomorphic to $W_{\alpha}$ by Lemma 8.6 and Equation (8.24) (with $V=V_{+, \alpha}$ ), and similarly for $D_{\Theta}$.

Lemma 8.16 shows that $\rho(X, \alpha, g)$ is independent of the choice of Riemannian metric on the interior of $X$ (as long as the metric is a product in some collar of the boundary) and the choice of flat connection $B$ with holonomy $\alpha$.

We now can state the main result of this section.

THEOREM 8.18. Suppose the closed manifold $M$ contains a hypersurface $N$ separating $M$ into $M^{+}$and $M^{-}$. Fix a Riemannian metric $g$ on $N$. Suppose that $\alpha: \pi_{1}(M) \rightarrow U(n)$ is a representation, and let $\tau: \pi_{1}(M) \rightarrow U(n)$ denote the trivial representation.

Then

$$
\rho(M, \alpha)=\rho\left(M^{+}, \alpha, g\right)+\rho\left(M^{-}, \alpha, g\right)+m\left(V_{+, \alpha}, V_{-, \alpha}, \alpha, g\right)-m\left(V_{+, \tau}, V_{-, \tau}, \tau, g\right) .
$$

Proof. This follows by applying Equation (8.32) to $B$ and $\Theta$ and subtracting.

It can be shown that the invariants $\rho\left(M^{ \pm}, \alpha, g\right)$ and $m\left(V_{+}, V_{-}, \alpha, g\right)$ depend in general on the choice of Riemannian metric $g$ on the hypersurface $N$. We leave an an intriguing open problem to determine exactly how they depend on the metric $g$, and in particular, how these invariants change if $g$ is replaced by the pulled-back metric $f^{*}(g)$ for a diffeomorphism $f: N \rightarrow N$.

8.3. Relationship to Wall's non-additivity theorem. Theorems 8.18 and 8.12 should be viewed as odd-dimensional counterparts of Wall's non-additivity theorem for the signature [32]. Indeed these theorems give formulas which express the "nonadditivity of the signature defect". The relationship between splitting theorems for the $\eta$-invariant and Wall non-additivity is explored in Bunke's article [9] and also in [20].

To clarify the relationship between Wall's theorem and Theorem 8.18, consider the following situation. Suppose we are given two $4 k$-dimensional manifolds $Z^{+}$and $Z^{-}$ 
$\eta$-INVARIANT, MASLOV INDEX, AND SPECTRAL FLOW

with $\partial Z^{ \pm}=M^{ \pm} \cup_{N} M^{0}$. Suppose that $\partial M^{0}=N$ and that $Z=Z^{+} \cup_{M^{0}} Z^{-}$. Finally suppose that $\alpha: \pi_{1} Z \rightarrow U(n)$ is a representation and let $\tau: \pi_{1} Z \rightarrow U(n)$ denote the trivial representation. The Atiyah-Patodi-Singer signature theorem [2, Theorem 2.4] says that

$$
\operatorname{Sign}_{\tau}(Z)-\operatorname{Sign}_{\alpha}(Z)=\rho(M, \alpha) .
$$

Similarly $\operatorname{Sign}_{\tau}\left(Z^{+}\right)-\operatorname{Sign}_{\alpha}\left(Z^{+}\right)=\rho\left(M^{+} \cup M^{0}, \alpha\right)$ and $\operatorname{Sign}_{\tau}\left(Z^{-}\right)-\operatorname{Sign}_{\alpha}\left(Z^{-}\right)=$ $\rho\left(-M^{0} \cup M^{-}, \alpha\right)$. On the other hand Wall's theorem says that

$$
\operatorname{Sign}_{\alpha}(Z)=\operatorname{Sign}_{\alpha}\left(Z^{+}\right)+\operatorname{Sign}_{\alpha}\left(Z^{-}\right)-\sigma\left(V_{+, \alpha}, V_{-, \alpha}, V_{0, \alpha}, \alpha\right),
$$

where $\sigma$ is a correction term which depends on the relative positions of the subspaces $V_{+, \alpha}, V_{-, \alpha}$ and $V_{0, \alpha}$ in $H^{*}\left(N ; \mathbb{C}_{\alpha}^{n}\right)$. Similarly $\operatorname{Sign}_{\tau}(Z)=\operatorname{Sign}_{\tau}\left(Z^{+}\right)+\operatorname{Sign}_{\tau}\left(Z^{-}\right)-$ $\sigma\left(V_{+, \tau}, V_{-, \tau}, V_{0, \tau}, \tau\right)$.

Hence

$$
\begin{aligned}
\sigma\left(V_{+, \alpha}, V_{-, \alpha}, V_{0, \alpha}, \alpha\right) & -\sigma\left(V_{+, \tau}, V_{-, \tau}, V_{0, \tau}, \tau\right) \\
= & \operatorname{Sign}_{\tau}(Z)-\operatorname{Sign}_{\tau}\left(Z^{+}\right)-\operatorname{Sign}_{\tau}\left(Z^{-}\right) \\
& -\left(\operatorname{Sign}_{\alpha}(Z)-\operatorname{Sign}_{\alpha}\left(Z^{+}\right)-\operatorname{Sign}_{\alpha}\left(Z^{-}\right)\right) \\
= & \rho\left(M^{+} \cup M^{-}, \alpha\right)-\rho\left(M^{+} \cup M^{0}, \alpha\right)-\rho\left(-M^{0} \cup M^{-}, \alpha\right)
\end{aligned}
$$

Applying Theorem 8.18 we see that (8.37) is equal to $\widetilde{\sigma}_{\alpha}-\widetilde{\sigma}_{\tau}$, where

$$
\tilde{\sigma}_{\alpha}:=m\left(V_{+, \alpha}, V_{-, \alpha}, \alpha, g\right)-m\left(V_{+, \alpha}, V_{0, \alpha}, \alpha, g\right)-m\left(V_{0, \alpha}, V_{-, \alpha}, \alpha, g\right)
$$

and

$$
\widetilde{\sigma}_{\tau}:=m\left(V_{+, \tau}, V_{-, \tau}, \tau, g\right)-m\left(V_{+, \tau}, V_{0, \tau}, \tau, g\right)-m\left(V_{0, \tau}, V_{-, \tau}, \tau, g\right) .
$$

This motivates introducing the following notation. Given a Hermitian symplectic space $H$, define the function of triples of Lagrangian subspaces

$$
\tilde{\sigma}_{H}: \mathscr{L}(H) \times \mathscr{L}(H) \times \mathscr{L}(H) \rightarrow \mathbb{Z}
$$

by

$$
\widetilde{\sigma}_{H}(V, W, U):=m_{H}(V, W)+m_{H}(W, U)+m_{H}(U, V) .
$$

By definition $\widetilde{\sigma}_{\alpha}=\widetilde{\sigma}_{H^{*}\left(N ; \mathbb{C}_{\alpha}^{n}\right)}\left(V_{+, \alpha}, V_{-, \alpha}, V_{0, \alpha}\right)$ and similarly for $\widetilde{\sigma}_{\tau}$. That $\tilde{\sigma}_{H}$ is an integer can be seen by exponentiating and using the multiplicativity of the determinant:

$$
\begin{aligned}
\exp \left(2 \pi i \widetilde{\sigma}_{H}(V, W, U)\right) & \\
=(\exp (\operatorname{tr} \log ( & \left.\left.-\phi(V) \phi(W)^{*}\right)+\operatorname{tr} \log \left(-\phi(W) \phi(U)^{*}\right)+\operatorname{tr} \log \left(-\phi(U) \phi(V)^{*}\right)\right)^{2} \\
& \left.=\operatorname{det}\left((-1)^{3} \phi(V) \phi(W)^{*} \phi(W) \phi(U)^{*} \phi(U) \phi(V)^{*}\right)^{2}\right) \\
& =1 .
\end{aligned}
$$

Proposition 8.19. The function $\widetilde{\sigma}_{H}$ satisfies the following properties.

1. Given a permutation $\beta, \widetilde{\sigma}_{H}\left(V_{\beta(1)}, V_{\beta(2)}, V_{\beta(3)}\right)=\operatorname{sign}(\beta) \widetilde{\sigma}_{H}\left(V_{1}, V_{2}, V_{3}\right)$.

2. $\widetilde{\sigma}_{H_{1} \oplus H_{2}}\left(V_{1} \oplus V_{2}, W_{1} \oplus W_{2}, U_{1} \oplus U_{2}\right)=\widetilde{\sigma}_{H_{1}}\left(V_{1}, W_{1}, U_{1}\right)+\widetilde{\sigma}_{H_{2}}\left(V_{2}, W_{2}, U_{2}\right)$. 
3. If $h: H \rightarrow H$ is a symplectic automorphism, then $\widetilde{\sigma}_{H}(h(V), h(W), h(U))=$ $\widetilde{\sigma}_{H}(V, W, U)$.

4. Take $H=\mathbb{C}^{2}$ with $\gamma=\left(\begin{array}{cc}0 & -1 \\ 1 & 0\end{array}\right)$. Then $\widetilde{\sigma}_{H}(\mathbb{C}(1,0), \mathbb{C}(1,1), \mathbb{C}(0,1))=1$.

Proof. The first and second assertions follow from the first and second assertions of Proposition 8.15.

For the third assertion, we first claim that the group $S p(H)$ of symplectic automorphisms of $H$ is path connected. To see this, fix a Lagrangian subspace $V$ of $H$. The map $S p(H) \rightarrow \mathscr{L}(H)$ taking $g$ to $g(V)$ is a fibration with fiber the subgroup $S p_{V}(H)$ consisting of those symplectic automorphisms which leave $V$ invariant. Next, $S p_{V}(H)$ fibers over $G L(V)$ by mapping $g \in S p_{V}(H)$ to the restriction $\left.g\right|_{V}$. The fiber $F$ of this map consists of those symplectic transformations $g$ so that $g$ restricts to the identity on $V$. Writing $H=V \oplus \gamma(V)$ it is easy to see that $F$ consists of all matrices of the form

$$
\left(\begin{array}{cc}
I & A \\
0 & I
\end{array}\right)
$$

with $A$ an arbitrary real matrix. Thus $F$ is contractible, and since $G L(V)$ is path connected, $S p_{V}(H)$ is also path connected. Finally, since $S p(H)$ fibers over the path connected space $\mathscr{L}(H) \cong U(n)$ with path connected fiber $S p_{V}(H)$, it is itself path connected.

Choose a path $h_{t}$ from the identity matrix to $h$. The third assertion of Proposition 8.15 shows that $m_{H}\left(h_{t}(V), h_{t}(W)\right)$ varies continuously in $t$. Thus the same is true of the integer-valued function $t \mapsto \widetilde{\sigma}_{H}\left(h_{t}(V), h_{t}(W), h_{t}(U)\right)$. Therefore this function is constant, completing the proof of the third assertion.

To prove the last fact, Notice that $\mathbb{C}^{2}=E_{i} \oplus E_{-i}$, where $E_{i}$ is the span of $(1,-i)$ and $E_{-i}$ is the span of $(1, i)$. It is easy to calculate that with respect to these bases,

$$
\phi(\mathbb{C}(1,0))=1, \phi(\mathbb{C}(1,1))=-i, \text { and } \phi(\mathbb{C}(0,1))=-1
$$

Thus

$$
\begin{aligned}
\widetilde{\sigma}_{H}(\mathbb{C}(1,0), \mathbb{C}(1,1), \mathbb{C}(0,1)) & =-\frac{1}{\pi i}(\log (-1 \cdot i)+\log (-(-i) \cdot(-1))+\log (-(-1)(1))) \\
& =1
\end{aligned}
$$

It follows from Proposition 8.19 and [10, Theorem 8.1] (suitably generalized to the complex Hermitian case) that $\widetilde{\sigma}_{H}$ is equal to the Maslov triple index $\tau_{H}$ defined in loc. cit. Therefore, $\widetilde{\sigma}_{H}$ depends only on the underlying symplectic form $\omega(x, y)=\langle x, \gamma y\rangle$, and not on the Hermitian metric. In particular $\widetilde{\sigma}_{\alpha}$ and $\tilde{\sigma}_{\tau}$ are independent of the Riemannian metric on $N$.

$\widetilde{\sigma}_{H}$ and the Maslov triple index $\tau_{\mu}$ defined in Section 6.2 are (of course) intimately related. $\tau_{\mu}$ is, up to normalization, what Bunke [9] called the twisted Maslov triple 
$\eta$-INVARIANT, MASLOV INDEX, AND SPECTRAL FLOW

index. A direct calculation shows the following:

$$
\begin{aligned}
\widetilde{\sigma}_{H}(V, W, U)= & -\tau_{\mu}(V, W, U)-\tau_{\mu}(\gamma V, W, U)-\tau_{\mu}(V, \gamma W, U) \\
& -\tau_{\mu}(V, W, \gamma U)+\operatorname{dim}(V \cap W)+\operatorname{dim}(W \cap U)+\operatorname{dim}(V \cap U), \\
\tau_{\mu}(V, W, U)= & \frac{1}{4}\left(\widetilde{\sigma}_{H}(V, W, U)-\widetilde{\sigma}_{H}(\gamma V, W, U)-\widetilde{\sigma}_{H}(V, \gamma W, U)\right. \\
& \left.-\widetilde{\sigma}_{H}(V, W, \gamma U)+2 \operatorname{dim}(\gamma V \cap W)+2 \operatorname{dim}(W \cap \gamma U)+2 \operatorname{dim}(V \cap \gamma U)\right) .
\end{aligned}
$$

Using Proposition 8.2 of loc. cit. we conclude that $\widetilde{\sigma}_{\alpha}$ equals Wall's correction term $\sigma\left(V_{+, \alpha}, V_{-, \alpha}, V_{0, \alpha}\right)$ and similarly $\tilde{\sigma}_{\tau}$ equals $\sigma\left(V_{+, \tau}, V_{-, \tau}, V_{0, \tau}\right)$.

Using the argument outlined in [20] one can analyze Wall's theorem using Theorem 8.12 as follows.

In the context described above, the Atiyah-Patodi-Singer signature theorem states that

$$
\operatorname{Sign}_{\alpha}(Z)=n \int_{Z} L-\eta\left(D_{B}, M^{+} \cup M^{-}\right),
$$

where $L$ denotes the $L$-polynomial of the Riemannian curvature tensor on $Z$. Similarly one obtains formulas for $\operatorname{Sign}_{\alpha}\left(Z^{ \pm}\right)$

$$
\operatorname{Sign}_{\alpha}\left(Z^{+}\right)=n \int_{Z^{+}} L-\eta\left(D_{B}, M^{+} \cup M^{0}\right)
$$

and

$$
\operatorname{Sign}_{\alpha}\left(Z^{-}\right)=n \int_{Z^{+}} L-\eta\left(D_{B},-M^{0} \cup M^{-}\right) .
$$

Applying Theorem 8.12 and using Lemma 6.9 as before, one obtains

$$
\operatorname{Sign}_{\alpha}(Z)-\operatorname{Sign}_{\alpha}\left(Z^{+}\right)-\operatorname{Sign}_{\alpha}\left(Z^{-}\right)=\widetilde{\sigma}_{\alpha}+n\left(\int_{Z} L-\int_{Z^{+}} L-\int_{Z^{-}} L\right) .
$$

At this point one can invoke Wall's theorem and the identification of $\widetilde{\sigma}_{\alpha}$ with Wall's correction term given above to conclude that the integrals cancel. (This is not immediate since the Riemannian metrics on $Z^{+}$and $Z^{-}$need to be in cylindrical form near the boundary to apply the Atiyah-Patodi-Singer theorem, but these do not glue to give a smooth metric on $Z$ in cylindrical form near the boundary.)

On the other hand, Wall's theorem can be proven by showing that the integrals cancel. This is discussed in [20] and so one obtains an analytic proof of Wall's theorem.

More importantly, Equation (8.38) establishes a direct relationship between the correction terms $\widetilde{\sigma}_{\alpha}$ and $\widetilde{\sigma}_{\tau}$ for the non-additivity of the signature to the correction term $m\left(V_{+, \alpha}, V_{-, \alpha}, \alpha, g\right)-m\left(V_{+, \tau}, V_{-, \tau}, \tau, g\right)$ for the non-additivity of the $\rho$ invariant.

8.4. Adiabatic stretching and general Dirac operators. Some of the preceding exposition for the odd signature operator extends to the more general context of arbitrary Dirac operators, and we discuss aspects of this now. The new feature of this approach is that the role of adiabatic stretching in the splitting formula for the $\eta$-invariant is clarified.

Suppose we are given an arbitrary Dirac operator $D$ on a split manifold $M=$ $M^{+} \cup_{N} M^{-}$. Assume as usual that $D=\gamma\left(\frac{d}{d x}+A\right)$ on a collar of $N$. Let $M_{r}$ denote 
the manifold obtained by replacing the collar $[-1,1] \times N$ of $N$ by the stretched collar $[-r, r] \times N$. Thus $M_{0}=M$. Given $0 \leq r<\infty$, let $L_{M^{ \pm}}^{r}$ denote the Cauchy data space of the operator $D$ acting on $M_{r}^{ \pm}=M^{ \pm} \cup([-r, 0] \times N)$, and let $L_{M^{ \pm}}^{\infty}$ denote the adiabatic limit $\lim _{r \rightarrow \infty} L_{M^{ \pm}}^{r}$. Lemma 3.2 of [14] states that the path $[0, \infty] \rightarrow \operatorname{Gr}(A)$ given by $r \mapsto L_{M^{ \pm}}^{r}$ is continuous.

We let $F_{\nu}^{ \pm}$denote the span of $\lambda$-eigenvectors of $A$ for $\pm \lambda>\nu$, and $E_{\nu}^{ \pm}$the span of $\lambda$-eigenvectors of $A$ for $0< \pm \lambda \leq \nu$, so that the $L^{2}$-sections over $N$ decompose as

$$
F_{\nu}^{-} \oplus E_{\nu}^{-} \oplus \operatorname{ker} A \oplus E_{\nu}^{+} \oplus F_{\nu}^{+}
$$

or, as a symplectic direct sum

$$
\left(F_{\nu}^{-} \oplus F_{\nu}^{+}\right) \oplus\left(E_{\nu}^{-} \oplus E_{\nu}^{+}\right) \oplus \operatorname{ker} A .
$$

Theorem 8.5 has a counterpart for general Dirac operators, but the conclusion is slightly weaker. The following theorem has a similar but simpler proof than Theorem 8.5. It is implied by Theorem 6.5 of [14.

THEOREM 8.20. Let $V_{+} \subset \operatorname{ker} A$ denote the limiting values of extended $L^{2}$ solutions on $M^{+}$, so $V_{+}=\operatorname{proj}_{\operatorname{ker} A}\left(L_{M^{+}} \cap\left(F^{-} \oplus \operatorname{ker} A\right)\right)$. Let $\nu \geq 0$ be a number in the nonresonance range of $D$, i.e. $L_{M^{+}} \cap F_{\nu}^{-}=0$. Then there exists a subspace $W_{+} \subset E_{\nu}^{-}$ isomorphic to the space of $L^{2}$ solutions to $D \beta=0$ on $M_{\infty}^{+}$so that letting $W_{+}^{\perp} \subset E_{\nu}^{-}$ denote the orthogonal complement of $W_{+}$in $E_{\nu}^{-}$,

$$
L_{M^{+}}^{\infty}=F_{\nu}^{+} \oplus\left(W_{+} \oplus \gamma\left(W_{+}^{\perp}\right)\right) \oplus V_{+}
$$

in the decomposition (8.41). Moreover, $\gamma\left(L_{M^{+}}^{\infty}\right) \cap L_{M^{+}}=0$.

Then we have the following theorem.

TheOREM 8.21. With notation as above, for any $r_{0} \geq 0$,

$$
\begin{aligned}
& \widetilde{\eta}\left(D, M_{r_{0}}\right)-\widetilde{\eta}\left(D, M^{+} ; L_{M^{+}}^{\infty}\right)-\widetilde{\eta}\left(D, M^{-} ; \gamma\left(L_{M^{+}}^{\infty}\right)\right) \\
& \quad=\operatorname{Mas}\left(L_{M^{-}}^{r}, L_{M^{+}}^{\infty}\right)_{r \in\left[r_{0}, \infty\right]}-\operatorname{Mas}\left(L_{M^{-}}^{r}, L_{M^{+}}^{r}\right)_{r \in\left[r_{0}, \infty\right]}
\end{aligned}
$$

REMARK 8.22. In light of Theorems 7.5 and 7.6 the term $\operatorname{Mas}\left(L_{M^{-}}^{r}, L_{M^{+}}^{r}\right)_{r \in\left[r_{0}, \infty\right]}$ in Theorem 8.21 can be thought of as the spectral flow of the family of operators on $M$ obtained by stretching the collar from $r_{0}$ to infinity. Similarly the term $\operatorname{Mas}\left(L_{M^{-}}^{r}, L_{M^{+}}^{\infty}\right)_{r \in\left[r_{0}, \infty\right]}$ can be thought of as the spectral flow of the family on $M^{-}$obtained by using the projection to $L_{M^{+}}^{\infty}$ as boundary conditions and stretching the collar of $M^{-}$from $r_{0}$ to infinity.

PROOF. We prove this for $r_{0}=0$, the general case is obtained by reparameterizing. Let $P_{t}$ denote the path of projections to the Cauchy data space $L_{M^{+}}^{r}$, where $t=1 /(r+1)$. Applying Theorem 5.9 and Proposition 5.1 to the path $P_{t}$ we see that $\widetilde{\eta}\left(D, M_{r_{0}}\right)-$ $\widetilde{\eta}\left(D, M^{+} ; L_{M^{+}}^{\infty}\right)-\widetilde{\eta}\left(D, M^{-} ; \gamma\left(L_{M^{+}}^{\infty}\right)\right)$ equals $\mathrm{SF}\left(D_{P_{t}}, M^{+}\right)+\mathrm{SF}\left(D_{I-P_{t}}, M^{-}\right)_{[0,1]}$, which by Theorem 7.5 equals

$$
\operatorname{Mas}\left(P_{t}, P_{M^{+}}\right)+\operatorname{Mas}\left(P_{M^{-}}, I-P_{t}\right) .
$$


Switching to Lagrangian notation and parameterizing by $r$ instead of $t$ we can rewrite (8.42) as

$$
-\operatorname{Mas}\left(\gamma\left(L_{M^{+}}^{r}\right), L_{M^{+}}\right)_{r \in[0, \infty]}-\operatorname{Mas}\left(L_{M^{-}}, L_{M^{+}}^{r}\right)_{r \in[0, \infty]}
$$

We use the homotopy invariance of the Maslov index to simplify these terms. Consider first $\operatorname{Mas}\left(\gamma\left(L_{M^{+}}^{r}\right), L_{M^{+}}\right)_{r \in[0, \infty]}$. We will show this term vanishes.

The path $r \mapsto \gamma\left(L_{M^{+}}^{r}\right)$ is homotopic to the composite of $r \mapsto \gamma\left(L_{M^{+}}^{r}\right)$ and the constant map at $\gamma\left(L_{M^{+}}^{\infty}\right)$, and the constant path at $L_{M^{+}}$is homotopic to the composite of $r \mapsto L_{M^{+}}^{r}$ and its inverse. Since $\gamma\left(L_{M^{+}}^{r}\right) \cap L_{M^{+}}^{r}=0$ for all $r, \operatorname{Mas}\left(\gamma\left(L_{M^{+}}^{r}\right), L_{M^{+}}^{r}\right)=0$ and so by path additivity of the Maslov index, $\operatorname{Mas}\left(\gamma\left(L_{M^{+}}^{r}\right), L_{M^{+}}\right)=-\operatorname{Mas}\left(\gamma\left(L_{M^{+}}^{\infty}\right), L_{M^{+}}^{r}\right)$. Theorem 8.20 says that $\gamma\left(L_{M^{+}}^{\infty}\right) \cap L_{M^{+}}^{r}=0$ for $r=0$, but by reparameterizing we see that the intersection is zero for all $r<\infty$; obviously $\gamma\left(L_{M^{+}}^{\infty}\right) \cap L_{M^{+}}^{\infty}=0$. Hence $\operatorname{Mas}\left(\gamma\left(L_{M^{+}}^{\infty}\right), L_{M^{+}}^{r}\right)=0$ and so

$$
\operatorname{Mas}\left(\gamma\left(L_{M^{+}}^{r}\right), L_{M^{+}}\right)_{r \in[0, \infty]}=0 .
$$

Consider now the term $\operatorname{Mas}\left(L_{M^{-}}, L_{M^{+}}^{r}\right)_{r \in[0, \infty]}$. The constant path at $L_{M^{-}}$is homotopic to the composite of $r \mapsto L_{M^{-}}^{r}$ and its inverse, and the path $r \mapsto L_{M^{+}}^{r}$ is homotopic to its composite with the constant path at $L_{M^{+}}^{\infty}$. Therefore,

$$
\operatorname{Mas}\left(L_{M^{-}}, L_{M^{+}}^{r}\right)=\operatorname{Mas}\left(L_{M^{-}}^{r}, L_{M^{+}}^{r}\right)-\operatorname{Mas}\left(L_{M^{-}}^{r}, L_{M^{+}}^{\infty}\right) .
$$

Substituting (8.44) and (8.45) into (8.43) finishes the proof.

We finish this article by outlining a few ways to use Theorem 8.21 to obtain other useful splitting formulas for the $\eta$-invariant. We will not give an exhaustive list, but we note that many other useful formulas can be derived from these using the results of Sections 5, 6, and 7. One can, of course, obtain other formulas by reversing the roles of $M^{+}$and $M^{-}$in Theorem 8.21 and in these examples.

ExAMPLE 8.23. Suppose that $L_{M^{-}}^{\infty} \cap L_{M^{+}}^{\infty}=0$. Then there exists an $r_{0} \geq 0$ so that $L_{M^{-}}^{r} \cap L_{M^{+}}^{r}=0$ and $L_{M^{-}}^{r} \cap L_{M^{+}}^{\infty}=0$ for all $r \geq r_{0}$. Applying Theorem 8.21 we see that if $r \geq r_{0}$ then

$$
\begin{aligned}
& \widetilde{\eta}\left(D, M_{r}\right)=\widetilde{\eta}\left(D, M^{+} ; L_{M^{+}}^{\infty}\right)+\widetilde{\eta}\left(D, M^{-} ; \gamma\left(L_{M^{+}}^{\infty}\right)\right) \\
& \quad=\widetilde{\eta}\left(D, M^{+} ; F_{\nu}^{+} \oplus W_{+} \oplus \gamma\left(W_{+}^{\perp}\right) \oplus V_{+}\right)+\widetilde{\eta}\left(D, M^{-} ; F_{\nu}^{-} \oplus W_{+}^{\perp} \oplus \gamma\left(W_{+}\right) \oplus \gamma\left(V_{+}\right)\right) .
\end{aligned}
$$

The hypothesis $L_{M^{-}}^{\infty} \cap L_{M^{+}}^{\infty}=0$ is a technically simpler replacement of the hypothesis "no exponentially small eigenvalues" which appears in related results in the literature.

ExAmPLE 8.24. Suppose that $D \beta=0$ has no $L^{2}$ solutions on $M_{\infty}^{+}$; i.e. that $W^{+}=0$ in Theorem 8.20. Then $L_{M^{+}}^{\infty}=F^{+} \oplus V_{+}$and so

$$
\begin{aligned}
& \widetilde{\eta}(D, M)-\widetilde{\eta}\left(D, M^{+} ; F^{+} \oplus V_{+}\right)-\widetilde{\eta}\left(D, M^{-} ; F^{-} \oplus \gamma\left(V_{+}\right)\right) \\
& =\operatorname{Mas}\left(L_{M^{-}}^{r}, F^{+} \oplus V_{+}\right)_{r \in[0, \infty]}-\operatorname{Mas}\left(L_{M^{-}}^{r}, L_{M^{+}}^{r}\right)_{r \in[0, \infty]}
\end{aligned}
$$

In other words, with respect to the Atiyah-Patodi-Singer boundary conditions given by the projection to $F^{+} \oplus V_{+}$on $M^{+}$and the projection to $F^{-} \oplus \gamma\left(V_{+}\right)$on $M^{-}$, the failure of the additivity of the $\widetilde{\eta}$-invariants is measured by $\operatorname{Mas}\left(L_{M^{-}}^{r}, F^{+} \oplus V_{+}\right)_{r \in[0, \infty]}-$ $\operatorname{Mas}\left(L_{M^{-}}^{r}, L_{M^{+}}^{r}\right)_{r \in[0, \infty]}$. As remarked above this is the difference of the spectral flow of 
$D$ on $M^{-}$with $F^{+} \oplus V_{+}$conditions as the collar of $M^{-}$is stretched to infinity, and the spectral flow of $D$ on $M$ as the collar is stretched to infinity.

EXAMPLE 8.25. We can combine the previous two examples as follows. Suppose that there are no $L^{2}$ solutions on $M_{\infty}^{+}$and $M_{\infty}^{-}$(i.e. $W_{+}=0=W_{-}$) and that the limiting values of extended $L^{2}$ solutions are transverse (i.e. $V_{+} \cap V_{-}=0$ in $\operatorname{ker} A$; this happens for example if $\operatorname{ker} A=0)$. Then $L_{M^{ \pm}}^{\infty}=F^{ \pm} \oplus V_{ \pm}$and so both of the previous examples apply.

Hence there exists an $r_{0} \geq 0$ so that $L_{M^{-}}^{r} \cap L_{M^{+}}^{r}=0$ and $L_{M^{-}}^{r} \cap L_{M^{+}}^{\infty}=0$ for all $r \geq r_{0}$. Therefore,

$$
\begin{aligned}
\widetilde{\eta}(D, M)-\widetilde{\eta}\left(D, M^{+}\right. & \left.; F^{+} \oplus V_{+}\right)-\widetilde{\eta}\left(D, M^{-} ; F^{-} \oplus \gamma\left(V_{+}\right)\right) \\
& =\operatorname{SF}\left(D, M_{r}^{-} ; F^{+} \oplus V_{+}\right)_{r \in\left[0, r_{0}\right]}-\operatorname{SF}\left(D, M_{r}\right)_{r \in\left[0, r_{0}\right]} .
\end{aligned}
$$

This says that the failure of additivity of the $\widetilde{\eta}$-invariants with Atiyah-Patodi-Singer boundary conditions is measured by the difference of the spectral flow of $D$ on $M^{-}$ with $F^{+} \oplus V_{+}$conditions as the length of the collar of $M^{-}$is stretched to $r_{0}$, and the spectral flow of $D$ on $M$ as the collar is stretched to $r_{0}$.

In particular, if $r \geq r_{0}$,

$$
\widetilde{\eta}\left(D, M_{r}\right)=\widetilde{\eta}\left(D, M^{+} ; F^{+} \oplus V_{+}\right)+\widetilde{\eta}\left(D, M^{-} ; F^{-} \oplus \gamma\left(V_{+}\right)\right) .
$$

This last formula appears in Bunke's article [9]. The reader should compare this formula with the formula of Theorem 8.8 (with $V=V_{+, \alpha}$ ) which, by contrast, holds in complete generality for the odd signature operator.

These examples, together with Theorem 8.20, underscore the point that difficulties in establishing simple splitting formulas for the $\eta$-invariant using Atiyah-Patodi-Singer boundary conditions arise from the existence of $L^{2}$ solutions on the two parts of the decomposition of $M$. To put this in a positive perspective, the failure of the additivity of the $\eta$-invariant with Atiyah-Patodi-Singer boundary conditions is measured by the spectral flow terms discussed in Remark 8.22 and symplectic invariants of the Lagrangian subspaces $W_{ \pm} \oplus \gamma\left(W_{ \pm}^{\perp}\right)$ in the finite-dimensional symplectic space $E_{\nu}^{-} \oplus E_{\nu}^{+}$ consisting of the span of the $\mu$-eigenvectors of $A$ with $-\nu \leq \mu \leq \nu, \mu \neq 0$. In our analysis of the odd signature operator the formulas simplify because we can control these terms; the spectral flow terms vanish for topological reasons and the symplectic invariants of the Lagrangian subspaces $W_{ \pm} \oplus \gamma\left(W_{ \pm}^{\perp}\right)$ vanish because of the additional control on $W_{ \pm}$that Theorem 8.5 provides over Theorem 8.20.

\section{References}

[1] M. F. Atiyah, V. K. Patodi, and I. M. Singer: Spectral asymmetry and Riemannian geometry I. Math. Proc. Camb. Phil. Soc. 77 (1975), 43-69

[2] M. F. Atiyah, V. K. Patodi, and I. M. Singer: Spectral asymmetry and Riemannian geometry II. Math. Proc. Camb. Phil. Soc. 78 (1975), 405-432

[3] J. Avron, R. Seiler, and B. Simon: The index of a pair of projections. J. Funct. Anal. 120 (1994), 220-237

[4] B. Booss-BavnbeK and K. Furutani: The Maslov index: a functional analytical definition and the spectral flow formula. Tokyo J. Math. 21 (1998), 1-34 
[5] B. Booss-Bavnbek and K. P. Wojciechowski: Elliptic Boundary Problems for Dirac Operators. Birkhäuser, Basel (1993)

[6] J. BRÜNING and M. LESCH: On boundary value problems for Dirac type operators: I. Regularity and self-adjointness. Preprint, 55p. (1999). Math.FA/9905181

[7] J. BRÜNING and M. LESCH: On the eta-invariant of certain non-local boundary value problems. Duke Math. J. 96 (1999), 425-468. Dg-ga/9609001

[8] J. BRÜNING and M. LESCH: Spectral theory of boundary value problems for Dirac type operators. In: B. Booß-Bavnbek and K. P. Wojciechowski (eds.), Geometric Aspects of PDE - Spectral Invariants (Roskilde Conf. Sept 1998), vol. 242 of Contemp. Math. AMS, Providence, RI (1999), pp. 203-215. Math.DG/9902100

[9] U. Bunke: On the gluing problem for the $\eta$-invariant. J. Diff. Geom. 41 (1995), 397-448

[10] S. Cappell, R. Lee, and E. Miller: On the Maslov index. Comm. Pure Appl. Math. 47 (1994), $121-186$

[11] S. Cappell, R. LeE, and E. Miller: Self-adjoint elliptic operators and manifold decompositions II: Spectral flow and Maslov index. Comm. Pure Appl. Math. 49 (1996), 869-909

[12] X. DAI and D. S. FreED: $\eta$-invariants and determinant lines. J. Math. Phys. 35 (1994), 51555194

[13] M. DaniEL: An extension of a theorem of Nicolaescu on spectral flow and the Maslov index. Proc. Amer. Math. Soc. 128 (2000), no. 2, 611-619

[14] M. DANiEL and P. KIRK: A general splitting formula for the spectral flow (with an appendix by K. P. Wojciechowski). Mich. Math. J. 46 (1999), 589-617

[15] R. G. Douglas and K. P. Wojciechowski: Adiabatic limits of the $\eta$-invariants the odddimensional Atiyah-Patodi-Singer problem. Commun. Math. Phys. 142 (1991), 139-168

[16] G. GrubB: Poles of zeta and eta functions for perturbations of the Atiyah-Patodi-Singer problem. University of Copenhagen, Preprint No. 14 (1999)

[17] G. GRubB: Trace expansions for pseudodifferential boundary problems for Dirac-type operators and more general systems. Arkiv f. Matematik 37 (1999), 45-86

[18] G. Grubb and R. Seeley: Weakly parametric pseudodifferential operators and Atiyah-PatodiSinger boundary problems. Invent. Math. 121 (1995), 481-529

[19] G. Grubb and R. T. Seeley: Zeta and eta functions for Atiyah-Patodi-Singer operators. J. Geom. Anal. 6 (1996), 31-77

[20] A. Hassell, R. Mazzeo, and R. Melrose: A signature formula for manifolds with corners of codimension two. Topology 36 (1997), no. 5, 1055-1075.

[21] T. Kato: Perturbation theory for linear operators, vol. 132 of Grundlehren der Mathematischen Wissenschaften. 2nd edn. Springer-Verlag, Berlin-Heidelberg-New York (1976)

[22] N. H. KUIPER: The homotopy type of the unitary group of Hilbert space. Topology 3 (1965), $19-30$

[23] M. LESCH: Determinants of Dirac type operators on manifolds with boundary. In preparation

[24] M. Lesch and K. P. WoJCIEChOWski: On the $\eta$-invariant of generalized Atiyah-Patodi-Singer boundary value problems. Ill. J. Math. 40 (1996), 30-46

[25] W. MüLLER: On the $L^{2}$-index of Dirac operators on manifolds with corners of codimension two. I. J. Diff. Geom. 44 (1996), 97-177

[26] L. Nicolaescu: The Maslov index, the spectral flow, and decompositions of manifolds. Duke Math. J. 80 (1995), 485-533

[27] R. S. Palais: Seminar on the Atiyah-Singer Index Theorem. Annals of Mathematics Studies, No. 57 Princeton University Press, Princeton, N.J. 1965 x+366 pp.

[28] J. PHILLIPS: Self-adjoint Fredholm operators and spectral flow. Canad. Math. Bull. 39 (1996), 460-467

[29] S. G. ScotT: Determinants of Dirac boundary value problems over odd-dimensional manifolds. Commun. Math. Phys. 173 (1995), 43-76 
[30] S. G. Scott and K. P. Wojciechowski: The $\zeta$-determinant and Quillen determinant for a Dirac operator on a manifold with boundary. Preprint, IUPUI (1999)

[31] R. T. SeEley: Topics in pseudo-differential operators. C.I.M.E., Conference on pseudodifferential operators 1968, Edizioni Cremonese, Roma, 1969, pp. 169-305

[32] C. T. C. WALL: Non-additivity of the signature. Invent. Math. 7 (1969), 269-274

[33] K. P. Wojciechowski: The additivity of the $\eta$-invariant. The case of an invertible tangential operator. Houston J. Math. 20 (1994), 603-621

[34] K. P. Wojciechowski: The additivity of the $\eta$-invariant. The case of a singular tangential operator. Commun. Math. Phys. 109 (1995), 315-327

[35] K. P. WoJciechowski: The $\zeta$-determinant and the additivity of the $\eta$-invariant on the smooth, self-adjoint Grassmannian. Commun. Math. Phys. 201 (1999), 423-444

Department of Mathematics, Indiana University, Bloomington, IN, 47405, USA

E-mail address: pkirk@indiana.edu

$U R L:$ http://php.indiana.edu/ pkirk

The University of Arizona, Department of Mathematics, 617 N. Santa Rita, Tucson, AZ, 85721-0089, USA

E-mail address: lesch@math.arizona.edu

$U R L:$ http://www.math.arizona.edu/ lesch 